\title{
STRONG RIGIDITY OF II 1 FACTORS ARISING FROM MALLEABLE ACTIONS OF $w$-RIGID GROUPS, II
}

\author{
SORIN POPA \\ University of California, Los Angeles
}

\begin{abstract}
We prove that any isomorphism $\theta: M_{0} \simeq M$ of group measure space $\mathrm{II}_{1}$ factors, $M_{0}=L^{\infty}\left(X_{0}, \mu_{0}\right) \rtimes_{\sigma_{0}} G_{0}, M=L^{\infty}(X, \mu) \rtimes_{\sigma} G$, with $G_{0}$ an ICC group containing an infinite normal subgroup with the relative property $(\mathrm{T})$ of KazhdanMargulis (i.e. $G_{0}$ w-rigid) and $\sigma$ a Bernoulli shift action of some group $G$, essentially comes from an isomorphism of probability spaces which conjugates the actions with respect to some identification $G_{0} \simeq G$. Moreover, any isomorphism $\theta$ of $M_{0}$ onto a "corner" $p M p$ of $M$, for $p \in M$ an idempotent, forces $p=1$. In particular, all group measure space factors associated with Bernoulli shift actions of w-rigid ICC groups have trivial fundamental group and any isomorphism of such factors comes from an isomorphism of the corresponding groups. This settles a "group measure space version" of Connes rigidity conjecture, shown in fact to hold true in a greater generality than just for ICC property $(\mathrm{T})$ groups. We apply these results to ergodic theory, establishing new strong rigidity and superrigidity results for orbit equivalence relations.
\end{abstract}

\section{INTRODUCTION.}

We continue in this paper the study of rigidity properties of isomorphisms $\theta: M_{0} \simeq M$ of crossed product $\mathrm{II}_{1}$ factors initiated in ([Po4]), concentrating here on the "group measure space" case, when the factors $M_{0}, M$ involved come from free ergodic measure preserving (m.p.) actions of groups on probability spaces. Similarly to ([Po4]), we assume the "source" factor $M_{0}$ comes from an arbitrary free ergodic measure preserving action $\sigma_{0}$ of a "mildly rigid" group $G_{0}$ (i.e. having a "large" subgroup with the relative property $(\mathrm{T})$ of Kazhdan-Margulis), while the "target" factor $M$ comes from an action $\sigma$ satisfying good "deformation+mixing"

Supported in part by a NSF Grant 0100883.

Typeset by $\mathcal{A} \mathcal{M} \mathcal{S}-\mathrm{TEX}_{\mathrm{E}}$ 
properties (typically a Bernoulli shift). Our main result shows that any isomorphism $\theta$ between such group measure space factors $M_{0}, M$ essentially comes from an isomorphism of probability spaces that conjugates the actions $\sigma_{0}, \sigma$, with respect to some isomorphism of the groups $G_{0}, G$. In particular, any isomorphism of the probability spaces that takes the orbits of $\sigma_{0}$ onto the orbits of $\sigma$ (almost everywhere), must come from a conjugacy of the actions $\sigma_{0}, \sigma$. This establishes new strong rigidity and superrigidity results in orbit equivalence ergodic theory, which are thus obtained through a von Neumann algebra approach.

The factors $M_{0}, M$ come from the following data: $\sigma_{0}: G_{0} \rightarrow \operatorname{Aut}\left(X_{0}, \mu_{0}\right)$, $\sigma: G \rightarrow \operatorname{Aut}(X, \mu)$ are free ergodic measure preserving (m.p) actions of countable discrete groups $G_{0}, G$ on standard probability spaces $\left(X_{0}, \mu_{0}\right),(X, \mu) ; M_{0}=$ $L^{\infty}\left(X_{0}, \mu_{0}\right) \rtimes_{\sigma_{0}} G_{0}, M=L^{\infty}(X, \mu) \rtimes_{\sigma} G$ are their associated group measure space (or crossed product) $\mathrm{II}_{1}$ factors, as defined by Murray and von Neumann ([MvN1]). In addition to being free and ergodic, $\left(\sigma_{0}, G_{0}\right),(\sigma, G)$ are assumed to satisfy:

(*). $G_{0}$ is w-rigid, i.e. it contains an infinite normal subgroup $H_{0}$ with the relative property $(\mathrm{T})$ of Kazhdan-Margulis (in other words, $\left(G_{0}, H_{0}\right)$ is a property $(\mathrm{T})$ pair, see [Ma], $[\mathrm{dHV}])$. We also consider the class $w \mathcal{T}_{0}$ of groups $G_{0}$ containing subgroups $H \subset G_{0}$ with the properties: $(a) .\left(G_{0}, H\right)$ is a property (T) pair; $(b) . H$ is not virtually abelian; $(c)$. $H$ satisfies a "very weak" normality condition in $G_{0}$ (see 7.0.3). The w-rigid groups will always be required ICC (infinite conjugacy class), while the groups in the class $w \mathcal{T}_{0}$ need not be ICC.

Infinite property $(\mathrm{T})$ groups are both w-rigid and in the class $w \mathcal{T}_{0}$. The groups $\mathbb{Z}^{2} \rtimes \Gamma$, for $\Gamma \subset S L(2, \mathbb{Z})$ non-amenable (cf [Ka], [Ma], [Bu]) and the groups $\mathbb{Z}^{N} \rtimes \Gamma$ for suitable actions of arithmetic lattices $\Gamma \subset S U(n, 1), S O(n, 1), n \geq 2$ (cf [Va], $[\mathrm{Fe}])$ are all w-rigid, but not in the class $w \mathcal{T}_{0}$. Any product of a w-rigid (resp. $w \mathcal{T}_{0}$ ) group with an arbitrary group is still w-rigid (resp. $w \mathcal{T}_{0}$ ).

$(* *) . \sigma$ is a Bernoulli shift action of an ICC group $G$. Thus, $\sigma$ acts on the probability space $(X, \mu)=\Pi_{g}\left(Y_{0}, \nu_{0}\right)_{g}$ by $\sigma_{g}\left(\left(x_{h}\right)_{h}\right)=\left(x_{h}^{\prime}\right)_{h}$, where $x_{h}^{\prime}=x_{g^{-1} h}, \forall h$. The key attributes of Bernoulli shifts that we actually need are: a deformation property called sub s-malleability (Definition 4.2 in [Po4]); a strong mixing property called clustering (Definition 1.1 in this paper). Thus, all statements below that require an action to be Bernoulli, equally work with the assumption "sub s-malleable and clustering".

The results we prove concern the structure of isomorphisms $\theta: M_{0} \simeq M$ between factors as above. More generally, we consider isomorphisms between amplifications of $M_{0}, M$ by positive real numbers ([MvN2]): If $N$ is a $\mathrm{II}_{1}$ factor and $t>0$ then the amplification of $N$ by $t$ is the (isomorphism class of the) $\mathrm{II}_{1}$ factor $N^{t} \stackrel{\text { def }}{=}$ 
$p M_{n \times n}(N) p$, where $n \geq t$ and $p$ is a projection in $M_{n \times n}(N)$ of normalized trace $\tau(p)$ equal to $t / n$. Thus, if $1 \geq t>0$ then $N^{t}$ is a "corner" $p N p$ of $N$, for $p \in N$ a projection of trace $t$.

Any orbit equivalence (OE) of $\sigma_{0}, \sigma$ (i.e. an isomorphism of probability spaces $\left(X_{0}, \nu_{0}\right) \simeq(X, \mu)$ which takes onto each other the equivalence relations $\mathcal{R}_{\sigma_{0}}, \mathcal{R}_{\sigma}$ given by the orbits of $\left.\left(\sigma_{0}, G_{0}\right),(\sigma, G)\right)$ implements an isomorphism of $M_{0}, M$ (cf. [Dy], $[\mathrm{FM}])$. More generally, if $Y \subset X$ has measure $\mu(Y)=t>0$ then any isomorphism $\left(X_{0}, \mu_{0}\right) \simeq\left(Y, \mu_{Y}\right)$ which takes $\mathcal{R}_{\sigma_{0}}$ onto the equivalence relation $\mathcal{R}_{\sigma}^{Y}$ obtained by intersecting the orbits of $\sigma$ with $Y$ implements an isomorphism $M_{0} \simeq M^{t}$. In general, not all isomorphisms of group measure space factors come from $\mathrm{OE}([\mathrm{CJ} 1])$, and $\mathrm{OE}$ need not imply isomorphism of groups ([MvN2], [Dy], $[\mathrm{OW}],[\mathrm{CFW}])$, much less conjugacy of actions. However, we have:

0.1. Theorem (vNE Strong Rigidity). Let $M_{0}, M$ be group measure space $\mathrm{II}_{1}$ factors arising from actions $\left(\sigma_{0}, G_{0}\right),(\sigma, G)$ satisfying $(*)$ resp. (**). If $\theta: M_{0} \simeq$ $M^{t}$ is an isomorphism of von Neumann algebras, for some $1 \geq t>0$, then $t=1$ and $\theta$ is of the form $\theta=\operatorname{Ad}(u) \circ \theta^{\gamma} \circ \theta^{\delta, \Delta}$, where: $u$ is a unitary element in $M$; $\theta^{\gamma} \in \operatorname{Aut}(M)$ is implemented by a character $\gamma$ of $G ; \theta^{\delta, \Delta}: M_{0} \simeq M$ is implemented by an isomorphism of groups $\delta: G_{0} \simeq G$ and an isomorphism of probability spaces $\Delta:\left(X_{0}, \mu_{0}\right) \simeq(X, \mu)$ satisfying $\sigma(\delta(h)) \circ \Delta=\Delta \circ \sigma_{0}(h), \forall h \in G_{0}$.

This extremely rigid situation is in sharp contrast with the amenable case, where by Connes' Theorem ([C3]) all group measure space $\mathrm{II}_{1}$ factors $L^{\infty}(X, \mu) \rtimes_{\sigma} G$ are isomorphic, and by results of Dye ([Dy]), Ornstein-Weiss ([OW]), Connes-FeldmanWeiss $([\mathrm{CFW}])$ all $(\sigma, G)$ are orbit equivalent. Instead, Theorem 0.1 is in line with the rigidity results established over the last 25 years in von Neumann algebra theory ([C1,2], [CJ2], [Po8], [CoHa], [GoNe], [Po1-4]) and orbit equivalence ergodic theory ([Zi], [GeGo], [Po8], [Ga1,2], [Fu1-3], [MoSh], [Hj], [PoSa], [Po9]).

In fact, the terminology "strong rigidity" is borowed from ergodic theory, where "strong orbit rigidity" ([Zi], [Fu1]), or "OE strong rigidity" ([MoSh]), designates results showing that within a certain class of group actions orbital equivalence automatically entails isomorphism of the groups and conjugacy of the actions. While deriving the same type of conclusion, Theorem 0.1 assumes an even weaker equivalence of actions than orbit equivalence (OE), namely von Neumann equivalence (vNE), i.e. isomorphism of the associated group measure space von Neumann algebras. Since Connes and Jones constructed examples of non-OE actions that give rise to isomorphic factors ([CJ1]), vNE is indeed (strictly) weaker than OE. Thus, Theorem 0.1 can be viewed both as a "strong rigidity"-type result in von Neumann algebra theory, and as a new, stronger rigidity statement for ergodic theory.

By applying this result to the case both actions are Bernoulli shifts and both 
groups are ICC and either w-rigid or in $w \mathcal{T}_{0}$, one obtains a large class of group measure factors that can be distinguished by the isomorphism class of their groupdata:

0.2. Corollary. Let $G_{i}$ be ICC and either w-rigid or in the class $w \mathcal{T}_{0}$ and $\sigma_{i}$ : $G_{i} \rightarrow \operatorname{Aut}\left(X_{i}, \mu_{i}\right)$ a Bernoulli $G_{i}$-action, $i=0,1$. Denote $M_{i}=L^{\infty}\left(X_{i}, \mu_{i}\right) \rtimes_{\sigma_{i}} G_{i}$ the associated group measure space $\mathrm{II}_{1}$ factors and $M_{i}^{\infty}=M_{i} \bar{\otimes} \mathcal{B}\left(\ell^{2} \mathbb{N}\right)$ the corresponding $\mathrm{II}_{\infty}$ factors, $i=0,1$. Then $M_{0}^{\infty} \simeq M_{1}^{\infty} \Leftrightarrow M_{0} \simeq M_{1} \Leftrightarrow \sigma_{0}$ conjugate to $\sigma_{1}$ with respect to some identification of $G_{0} \simeq G$. Even more so, if $\theta: M_{0}^{\infty} \simeq M_{1}^{\infty}$, then $\theta$ is the amplification of an isomorphism $\theta_{0}: M_{0} \simeq M_{1}$ of the form $\theta_{0}=\operatorname{Ad}(u) \circ \theta^{\gamma} \circ \theta^{\delta, \Delta}$, for some $u \in \mathcal{U}\left(M_{1}\right), \gamma \in \operatorname{Char}\left(G_{1}\right)$ and $\delta: G_{0} \simeq G_{1}$, $\Delta:\left(X_{0}, \mu_{0}\right) \simeq\left(X_{1}, \mu_{1}\right)$ satisfying $\sigma_{1}(\delta(h)) \Delta=\Delta \sigma_{0}(h), \forall h \in G_{0}$.

At the Kingston AMS Summer School in 1980 A. Connes posed the problem of showing that factors arising from property (T) ICC groups are isomorphic iff the groups are isomorphic (see [C2]). He formulated the question related to his discovery that property (T) ICC groups $G$ give rise to group factors $L(G)$ with rigid symmetry structure $([\mathrm{C} 1])$. By now, several results in von Neumann algebras ([CoHa], [Po8], [CSh]) and ergodic theory ([Zi], [CoZi], [GeGo], [GoNa], [Fu1,2]) provide supporting evidence towards a positive answer to the conjecture (see V.F.R. Jones' comments on the "higher rank lattice" version of this problem in [J2]).

Since $L^{\infty}\left(X_{i}, \mu_{i}\right) \rtimes_{\sigma_{i}} G_{i}$, for $\sigma_{i}$ Bernoulli, can be viewed as a canonical "group measure space"-version of the group factor $L\left(G_{i}\right)$, Corollary 2 can be regarded as an affirmative answer to a natural "relative variant" of the conjecture. This is best emphasized by re-formulating Corollary 0.2 as a "group factor" statement, by using wreath product groups. Thus, if we denote $\tilde{G}=\mathbb{Z}^{G} \rtimes G$ (the wreath product with "base" $\mathbb{Z}$ ) then the group factor $L(\tilde{G})$ is naturally isomorphic to the group measure space factor corresponding to the Bernoulli shift action $\sigma$ of $G$ on $\mathbb{T}^{G}$, and so we get:

0.3. Corollary. Let $G_{i}$ be w-rigid ICC groups and denote $\tilde{G}_{i}=\mathbb{Z}^{G_{i}} \rtimes G_{i}$ the corresponding wreath product, $i=0,1$. Then $L\left(\tilde{G}_{0}\right) \simeq L\left(\tilde{G}_{1}\right)$ iff $\tilde{G}_{0} \simeq \tilde{G}_{1}$ and iff $G_{0} \simeq G_{1}$.

Corollary 0.2 shows that all group measure space factors $L^{\infty}(X, \mu) \rtimes_{\sigma} G$ for $G$ w-rigid ICC and $\sigma$ Bernoulli $G$-action have trivial fundamental group. It also reduces the calculation of the outer automorphism group of such a factor to the calculation of the commutant of $\sigma$ in the set of automorphisms of the probability space $(X, \mu), \operatorname{Aut}_{0}(\sigma, G) \stackrel{\text { def }}{=} \sigma(G)^{\prime} \cap \operatorname{Aut}(X, \mu)$ :

0.4. Corollary. Let $G$ be $I C C$ and either $w$-rigid or in $w \mathcal{T}_{0}$. If $\sigma: G \rightarrow \operatorname{Aut}(X, \mu)$ is a Bernoulli $G$-action and $M=L^{\infty}(X, \mu) \rtimes_{\sigma} G, M^{\infty}=M \bar{\otimes} \mathcal{B}\left(\ell^{2} \mathbb{N}\right)$ then $M$ has 
trivial fundamental group, $\mathscr{F}(M)=\{1\}$, and $\operatorname{Out}\left(M^{\infty}\right)=\operatorname{Out}(M)=\operatorname{Aut}_{0}(\sigma, G) \times$ $(\operatorname{Char}(G) \rtimes \operatorname{Out}(G))$. Similarly, $\mathscr{F}\left(\mathcal{R}_{\sigma}\right)=\{1\}, \operatorname{Out}\left(\mathcal{R}_{\sigma}\right)=\operatorname{Aut}_{0}(\sigma, G) \times \operatorname{Out}(G)$.

The first examples of $\mathrm{II}_{1}$ factors with trivial fundamental group were obtained in ([Po2]; see also [Po3]). The key result which allowed this computation was a "strong rigidity"-type result similar to Theorem 0.1, showing that any stable vNE of two actions $\left(\sigma_{0}, G_{0}\right),(\sigma, G)$, with $\sigma_{0}$ satisfying a relative property $(\mathrm{T})$ in the spirit of Kazhdan-Margulis and Connes-Jones ([CJ2]), and with $G$ satisfying Haagerup's compact approximation property, comes from an $\mathrm{OE}$ of the actions. Thus, for the class $\mathcal{H} \mathcal{T}$ of factors arising from actions satisfying both properties, all OE invariants (such as Gaboriau's cost or $\ell^{2}$-Betti numbers $[$ Ga1,2]) are in fact isomorphism invariants of the factors. Using the calculations of $\ell^{2}$-Betti numbers in $([\mathrm{Ga} 2])$ one derives that $L^{\infty}\left(\mathbb{T}^{2}, \lambda\right) \rtimes \mathbb{F}_{n}=L\left(\mathbb{Z}^{2} \rtimes \mathbb{F}_{n}\right)$ have trivial fundamental group and are mutually non-isomorphic for $n=2,3, \ldots$. Thus, the approach for calculating fundamental groups in ([Po2]) makes crucial use of Gaboriau's work in OE ergodic theory.

In turn, the approach in this paper leads to a direct calculation of fundamental group of factors, through purely von Neumann algebra techniques, without using OE ergodic theory. Even more so, it provides new calculations of fundamental groups of equivalence relations, not covered by ([Ga1,2], [Fu1,2], [MoSh]).

We already pointed out that Theorem 0.1 implicitly gives an OE strong rigidity result. We mention two more OE rigidity results that we can derive from 0.1 (see Section 7 for more applications). The first one concerns embeddings of equivalence relations and it can be viewed as a Galois-type correspondence between w-rigid subequivalence relations of a Bernoulli $G$-action and w-rigid subgroups of $G$ :

0.5. Theorem (OE Strong Rigidity for Embeddings). Let $\sigma: G \rightarrow \operatorname{Aut}(X, \mu)$ be a Bernoulli shift action of an ICC group $G$. If $\sigma_{0}$ is a free ergodic m.p. action of a w-rigid ICC group $G_{0}$ (or of a group $G_{0} \in w \mathcal{T}_{0}$ ) on $\left(X_{0}, \mu_{0}\right)$ and $\Delta_{0}:\left(X_{0}, \mu_{0}\right) \simeq$ $(X, \mu)$ satisfies $\Delta_{0}\left(\mathcal{R}_{\sigma_{0}}\right) \subset \mathcal{R}_{\sigma}$ then there exists an isomorphism $\delta: G_{0} \simeq G_{0}^{\prime} \subset G$ and $\alpha \in \operatorname{Inn}\left(\mathcal{R}_{\sigma}\right)$ such that $\Delta=\alpha \circ \Delta_{0}$ satisfies $\Delta \circ \sigma_{0}(h)=\sigma(\delta(h)) \Delta, \forall h \in G_{0}$.

If we restrict ourselves from w-rigid to Kazhdan groups, one can deduce from Theorem 0.1 an "OE rigidity" result where all conditions on the actions and on the groups involved are on just "one side", a type of result labelled "OE superrigidity" in $([\mathrm{MoSh}])$ :

0.6. Theorem (OE Superrigidity). Let $G$ be an ICC property $(\mathrm{T})$ group and $\sigma: G \rightarrow \operatorname{Aut}(X, \mu)$ a Bernoulli shift action. Let $G_{0}$ be any group and $\sigma_{0}: G_{0} \rightarrow$ $\operatorname{Aut}\left(X_{0}, \mu_{0}\right)$ any free m.p. action of $G_{0}$. If $\Delta_{0}:\left(X_{0}, \mu_{0}\right) \simeq\left(Y, \mu_{\mid Y}\right)$ satisfies $\Delta_{0}\left(\mathcal{R}_{\sigma_{0}}\right)=\mathcal{R}_{\sigma}^{Y}$, for some subset $Y \subset X$ of positive measure, then $Y=X$ and 
there exist $\alpha \in \operatorname{Inn}\left(\mathcal{R}_{\sigma}\right), \delta: G_{0} \simeq G, \Delta:\left(X_{0}, \mu_{0}\right) \simeq(X, \mu)$ satisfying $\sigma(\delta(h)) \Delta=$ $\Delta \sigma_{0}(h), \forall h \in G_{0}$, such that $\Delta_{0}=\alpha \circ \Delta$. In particular, if $Y \subset X$ has measure $0 \neq \mu(Y)<1$ then the equivalence relation $\mathcal{R}_{\sigma}^{Y}$ cannot be realized as orbits of a free action of a group.

OE rigidity results were pioneered by Zimmer in the early 80's (see [Zi]). Furman revived this direction, establishing further, sweeping OE rigidity results for classic actions of higher rank lattices ([Fu1]). Most recently, Monod and Shalom proved surprising "OE strong rigidity and superrigidity" results for a completely new class of groups (e.g. products of torsion free non-elementary hyperbolic groups) under very general ergodicity conditions on the actions ([MoSh]). The above applications bring additional insight to this fascinating subject.

A few words on the proofs: It is the sub s-malleability ( + mixing) condition on $\sigma$ and the "weak property (T)" (w-rigidity) of $G_{0}$ that were used in ([Po4]) to prove that any $\theta: M_{0} \simeq p M p$ as in Theorem 0.1 can be perturbed by an inner automorphism of $p M p$ so that to take the group von Neumann algebra $L\left(G_{0}\right)$, generated by $G_{0}$ in $M_{0}$, into a corner of the group von Neumann algebra $L(G)$, generated by $G$ in $M$. This preliminary rigidity result is crucial for the analysis in this paper. It reduces the proof of Theorem 0.1 to settling the case $M_{0}=p M p$, $p \in L(G), L\left(G_{0}\right) \subset p L(G) p$. We in fact prove a much more general statement, which only requires $\sigma$ clustering, letting $G, G_{0}, \sigma_{0}$ arbitrary:

0.7. Theorem (Criterion for Conjugacy of Actions). Let $\sigma: G \rightarrow \operatorname{Aut}(X, \mu)$, $\sigma_{0}: G_{0} \rightarrow \operatorname{Aut}\left(X_{0}, \mu_{0}\right)$ be free, ergodic, m.p. actions. Denote $A=L^{\infty}(X, \mu)$, $M=A \rtimes_{\sigma} G, A_{0}=L^{\infty}\left(X_{0}, \mu_{0}\right), M_{0}=A_{0} \rtimes_{\sigma_{0}} G_{0}$. Let $L(G) \subset M$ (respectively $\left.L\left(G_{0}\right) \subset M_{0}\right)$ be the von Neumann subalgebra generated by the canonical unitaries $\left\{u_{g}\right\}_{g \in G} \subset M$ implementing the action $\sigma$ (resp. $\left\{u_{h}^{0}\right\}_{h \in G_{0}} \subset M_{0}$ implementing $\left.\sigma_{0}\right)$. Let also $p \in L(G)$ be a projection and assume:

(a). $\sigma$ is clustering.

(b). $p M p=M_{0}$, with $L\left(G_{0}\right)$ contained in $p L(G) p$.

Then $p$ is central in $L(G), \tau(p)^{-1}$ is an integer, $p L(G) p=L\left(G_{0}\right)$ and there exist a normal subgroup $K \subset G$ with $|K|=\tau(p)^{-1}$, an $\operatorname{Ad}(G)$-invariant character $\kappa$ of $K$, implementing a trivial 2-cocycle on $G / K$, and a unitary element $u \in p L(G) p$ such that:

(i). $p=|K|^{-1} \Sigma_{k \in K} \kappa(k) u_{k}$ and $u_{k} p=\kappa(k) p, \forall k \in K$.

(ii). $u A_{0} u^{*}=A^{K} p$, where $A^{K}=\left\{a \in A \mid \sigma_{k}(a)=a, \forall k \in K\right\}$.

(iii). $\left\{u_{g} p \mid g \in G\right\}=\left\{u u_{h}^{0} u^{*} \mid h \in G_{0}\right\}$, modulo multiplication by scalars. More precisely, there exist an isomorphism $\delta: G_{0} \simeq G / K$, a lifting $G / K \ni g \mapsto g^{\prime} \in G$ and a map $\alpha: G_{0} \rightarrow \mathbb{T}$ such that $\operatorname{Ad}(u)\left(u_{h}^{0}\right)=\alpha(h) u_{\delta(h)^{\prime}} p, \forall h \in G_{0}$. 
If in addition $G$ is assumed ICC then $K=\{e\}, p=1$ and $\delta$ is an isomorphism $\delta: G_{0} \simeq G$.

Note that this result holds true even in the hyperfinite case, i.e. when $G_{0}, G$ are amenable. In fact, if we apply it to the particular case $M_{0}=M=R, G=G_{0}$ abelian, $p=1$ and $L(G)=L\left(G_{0}\right)$ then conclusion $(i i)$ alone gives an affirmative answer to a recent conjecture of Neshveyev and Størmer in ([NeSt]), but for the more restrictive class of clustering actions (e.g. Bernoulli shifts) rather than just weakly mixing, as formulated in ([NeSt]).

This Conjugacy Criterion is stated as Theorem 5.1 in the text. Its proof, which occupies most of the paper (sections 1-6), splits into two parts, using rather different techniques: Part I consists in proving a "Cartan Conjugacy Criterion" (Theorem 4.2 in the text), showing that assumptions $(a),(b)$ above imply $A_{0}$ can be unitary conjugate onto a corner of $A$. The proof, which takes Sections 1-4, utilises (A.1 in [Po2]), ultrapower algebra techniques and a careful "asymptotic analysis" of Fourier expansions $\Sigma_{g} a_{g} u_{g}$ for elements in $A \rtimes_{\sigma} G$. It is the only part that uses the clustering assumption on $\sigma$ (see 1.1 below for the definition of clustering).

Part II of the proof of 0.7 consists in showing that if in addition to $(a),(b)$ we also assume $A_{0}$ can be unitary conjugate onto a corner of $A$, then $(i)-($ iii $)$ follow. This is Theorem 5.2 in the text. In fact, instead of $(a)$ (i.e. the clustering condition on $\sigma$ ), for this result we only assume $\sigma$ and $\sigma_{0}$ mixing. The proof takes Sections 5 and 6 and uses "local quantization" techniques, in the spirit of (A.1 in [Po9]).

Our results leave unsolved the problem of showing that a group measure space factor arising from a Bernoulli action of an ICC property (T) group has ALL its Cartan subalgebras unitary conjugate. Or at least showing that an isomorphism of group measure space factors $M_{0}, M$ arising from actions $\left(\sigma_{0}, G_{0}\right),(\sigma, G)$ is sufficient to imply that if $G$ has $(\mathrm{T})$ then $G_{0}$ has $(\mathrm{T})$. If true, then Theorem 0.1 would also imply a "vNE superrigidity"-type result for factors, similar to the OE superrigidity in Theorem 0.6.

The present article is an outgrowth of a paper with the same title and references (math.OA/0407137) that we have circulated since July 2004 and in which we only proved that an isomorphism $\theta: M_{0} \simeq M^{t}$ with $M_{0}, M$ satisfying $(*),(* *)$ comes from an orbit equivalence of $\sigma_{0}, \sigma^{t}$. To derive from that the triviality of the fundamental group and rigidity results for factors, we had to make additional restrictions on the groups and rely on OE rigidity results of Gaboriau ([Ga]), Monod-Shalom $([\mathrm{MoSh}])$ and $([\mathrm{PoSa}])$. All these applications are now consequences of the "vNE Strong Rigidity" (0.1) and are shown is a greater generality. 


\section{Clustering properties for aCtions}

The purpose of the first three sections is to lay down the necessary technical background for the proof of the Cartan Conjugacy Criteria in Section 4.

Thus, in this Section we define the "clustering" property for actions of groups and a related notion of "clustering coefficients" for sequences of elements in the corresponding cross-product algebras. The considerations we make in this Section, as well as in Sections 2 and part of 3, concern actions of groups by trace preserving automorphisms on arbitrary finite von Neumann algebras $(N, \tau)$ ( $\tau$ denoting the fixed faithful normal trace on $N$ ). Starting with 3.5 though, the finite von Neumann algebra $(N, \tau)$ will always be abelian, a fact that will be emphasized by using the generic notation $A$ instead of $N$ (possibly with indices).

1.1. Definition. Let $\sigma: G \rightarrow \operatorname{Aut}(N, \tau)$ be an action of a discrete group $G$ on a finite von Neumann algebra $(N, \tau)$. A pair $\left(N^{0},\left\{B_{n}\right\}_{n}\right)$ consisting of a dense *subalgebra $N^{0} \subset N$ and a decreasing sequence of von Neumann subalgebras $\left\{B_{n}\right\}_{n}$ of $N$ is a clustering resolution for $\sigma$ if it satisfies the following conditions:

(1.1.1). For all $y \in N^{0}$ there exists $m$ such that $E_{B_{m}}(y)=\tau(y) 1$;

(1.1.2). For all $m$ and all $y \in N^{0}$ there exists $F \subset G$ finite such that $\sigma_{g}(y) \in B_{m}$, $\forall g \in G \backslash F$;

(1.1.3). For all $m \geq 1$ and all $g \in G$ there exists $n$ such that $\sigma_{g}\left(B_{n}\right) \subset B_{m}$.

An action $\sigma$ is clustering if it has a clustering resolution.

1.2. Proposition. $1^{\circ}$. If $\sigma$ is clustering then for any $n \geq 1$ it satisfies the following n-mixing condition:

$$
\lim _{g_{1}, \ldots, g_{n} \rightarrow \infty}\left|\tau\left(y_{0} \sigma_{g_{1}}\left(y_{1}\right) \ldots \sigma_{g_{n}}\left(y_{n}\right)\right)-\tau\left(y_{0}\right) \tau\left(\sigma_{g_{1}}\left(y_{1}\right) \ldots \sigma_{g_{n}}\left(y_{n}\right)\right)\right|=0
$$

for all $y_{0}, y_{1}, \ldots, y_{n} \in N$.

$2^{\circ}$. If $\sigma$ is either a commutative or a Connes-Størmer Bernoulli shift action then $\sigma$ is clustering.

Proof. $1^{\circ}$. By (1.1.1), and Kaplansky's density theorem, $\lim _{n \rightarrow \infty}\left\|E_{B_{n}}(x)-\tau(x) 1\right\|_{2}=$ $0, \forall x \in N$. Thus, for all $x \in N$ we have $\lim _{n \rightarrow \infty}\left|\tau\left(E_{B_{n}}(x) x^{\prime}\right)-\tau(x) \tau\left(x^{\prime}\right)\right|=0$ uniformly in $x^{\prime} \in(N)_{1}$. Letting $x=y_{0}, \stackrel{n \rightarrow \infty}{=} \sigma_{g_{1}}\left(y_{1}\right) \ldots \sigma_{g_{n}}\left(y_{n}\right)$ and applying (1.1.2), it follows that

$$
\lim _{g_{1}, \ldots, g_{n} \rightarrow \infty} \tau\left(y_{0} \sigma_{g_{1}}\left(y_{1}\right) \ldots \sigma_{g_{n}}\left(y_{n}\right)\right)=\tau\left(y_{0}\right) \tau\left(\sigma_{g_{1}}\left(y_{1}\right) \ldots \sigma_{g_{n}}\left(y_{n}\right)\right)
$$


$2^{\circ}$. Let $\left(\mathcal{N}_{0}, \varphi_{0}\right)$ be a von Neumann algebra with discrete decomposition. Assume $\sigma$ is the $\left(\mathcal{N}_{0}, \varphi_{0}\right)$-Bernoulli shift action of $G$ on $(\mathcal{N}, \varphi)=\underset{g \in G}{\otimes}\left(\mathcal{N}_{0}, \varphi_{0}\right)_{g}$ given by $\sigma_{h}\left(\otimes_{g} x_{g}\right)=\otimes_{g} x_{g}^{\prime}$, where $x_{g}^{\prime}=x_{h^{-1} g}$. It also acts on the centralizer algebra $N=\mathcal{N}_{\varphi}$, by restriction.

Let $S_{n}$ be an increasing sequence of finite subsets of $G$ such that $\cup_{n} S_{n}=G$ and denote by $\mathcal{B}_{n}=\bar{\otimes}_{g \in S_{n}^{c}}\left(\mathcal{N}_{0}, \varphi_{0}\right)_{g}$, where $S_{n}^{c}=G \backslash S_{n}$. We claim that $N^{0}=$ $\mathcal{N}_{\varphi} \cap \otimes_{g}\left(\mathcal{N}_{0}, \varphi_{0}\right)_{g} \subset N$ (algebraic tensor product) and $B_{n}=\mathcal{N}_{\varphi} \cap \mathcal{B}_{n}, n \geq 1$, give a clustering resolution for the action $\sigma$ of $G$ on $N=\mathcal{N}_{\varphi}$. Indeed, condition (1.1.1) is clearly satisfied by the definition and by the fact that $g h \in S_{n}^{c}$ as $g \rightarrow \infty, \forall h \in G$.

To check (1.1.2) note that if $y \in \mathcal{N}_{\varphi} \cap \overline{\otimes_{g \in S_{n}}}\left(\mathcal{N}_{0}, \varphi_{0}\right)_{g}$, for some large enough $n$, then for any $m, \sigma_{g}(y) \in B_{m}$ as $g \rightarrow \infty$.

To show that (1.1.3) is verified, consider the weakly dense subalgebra $B_{n}^{0}=$ $B_{n} \cap N^{0}$ of $B_{n}$ and note that since $\cup_{n} S_{n}=G$, we have $g^{-1} S_{k} \subset S_{n}$ (equivalently $\left.g\left(S_{n}^{c}\right) \subset S_{k}^{c}\right)$ as $n \rightarrow \infty$. This implies that $\sigma_{g}\left(B_{n}^{0}\right) \subset B_{k}$ as $n \rightarrow \infty$. But for each $n$ for which we have the above inclusion we also have $\sigma_{g}\left(B_{n}\right) \subset B_{k}$. Q.E.D.

We denote by $M=N \rtimes_{\sigma} G$ the cross-product von Neumann algebra associated to an action $\sigma: G \rightarrow \operatorname{Aut}(N, \tau)$ and by $\left\{u_{g}\right\}_{g} \subset M$ the canonical unitaries implementing the action $\sigma$. Also, we denote by $L(G)$ the von Neumann subalgebra of $M$ generated by the unitaries $\left\{u_{g}\right\}_{g}$.

Let $\omega$ be a free ultrafilter on $\mathbb{N}$, also viewed as a point in the Stone-Cech compactification of $\mathbb{N}, \omega \in \overline{\mathbb{N}} \backslash \mathbb{N}$. Note that a subset $V \subset \mathbb{N}$ in the ultrafilter $\omega$ corresponds in this latter case to a neighborhood of the point $\omega$ in the compact set $\overline{\mathbb{N}}$.

As usual, given a finite von Neumann algebra $(B, \tau)$ we denote by $\left(B^{\omega}, \tau\right)$ its ultrapower algebra, i.e. the finite von Neumann algebra $\ell^{\infty}(\mathbb{N}, B) / \mathcal{I}_{\omega}$, where $\mathcal{I}_{\omega}$ is the ideal of elements $x=\left(x_{n}\right)_{n} \in \ell^{\infty}(\mathbb{N}, B)$ with $\tau\left(x^{*} x\right)=0$, the trace $\tau=\tau_{\omega}$ on $\ell^{\infty}(\mathbb{N}, B) / \mathcal{I}_{\omega}$ being defined by $\tau\left(\left(b_{n}\right)_{n}\right)=\lim _{n \rightarrow \omega} \tau\left(b_{n}\right)=0$. For basic properties and results on ultrapower algebras see $([\mathrm{McD}])$.

We often identify $B$ with the subalgebra of constant sequences in $B^{\omega}$.

1.3. Definition. Let $\sigma: G \rightarrow \operatorname{Aut}(N, \tau)$ be a properly outer action with clustering resolution $\left(N^{0},\left\{B_{k}\right\}_{k}\right)$. A sequence $\left(x_{n}\right)_{n} \in \ell^{\infty}(\mathbb{N}, M)$ has clustering coefficients with respect to $\left(N^{0},\left\{B_{n}\right\}_{n}\right)$ (and to $\omega$ ) if $\forall \varepsilon>0$ and $\forall m \geq 1, \exists V \in \omega$ such that if $e_{k}$ denotes the orthogonal projection of $L^{2}(M)$ onto $L^{2}\left(\Sigma_{g} B_{k} u_{g}\right)$ then $\| e_{m}\left(\hat{x}_{n}\right)-$ $\hat{x}_{n} \|_{2} \leq \varepsilon, \forall n \in V$. Note that if $x=\left(x_{n}\right)_{n}$ has clustering coefficients then any element in $x+\mathcal{I}_{\omega}$ also has clustering coefficients. Thus, this property passes to elements in $M^{\omega}=\ell^{\infty}(\mathbb{N}, M) / I_{\omega}$. 
1.3.' Notations. We denote by $\mathcal{X}$ the set of sequences $\left(x_{n}\right)_{n} \in \ell^{\infty}(\mathbb{N}, M)$ with clustering coefficients. We still denote by $\mathcal{X}$ the image in $M^{\omega}$ of the set of sequences with clustering coefficients. Also, we denote by $\mathcal{Y}$ the set of elements $\left(x_{n}\right)_{n}$ in $M^{\omega}$ with the property that $\lim _{n \rightarrow \omega}\left\|E_{N}\left(x_{n} u_{g}\right)\right\|_{2}=0, \forall g \in G$.

We denote by $N^{\omega} \rtimes_{\sigma} G$ the von Neumann subalgebra of $M^{\omega}$ generated by $N^{\omega}$ and the canonical unitaries $u_{g}, g \in G$ (regarded as constant sequences in $M^{\omega}$ ). Also, we view the group von Neumann algebra $L(G)=\left\{u_{g}\right\}_{g}^{\prime \prime}$ as a subalgebra of $M^{\omega}$, via the inclusion $M \subset M^{\omega}$.

1.4. Lemma. $1^{\circ} \cdot \mathcal{X}, \mathcal{Y}$ are vector spaces.

$2^{\circ}$. If $\left\{x^{k}\right\}_{k} \subset \mathcal{X}$ is convergent in the norm \|\|$_{2}$ to an element $x \in M^{\omega}$ then $x \in \mathcal{X}$. Also, the unit ball of $\mathcal{X}$ (resp. $\mathcal{Y})$ is complete in the norm \|\|$_{2}$ given by the trace $\tau$ on $M^{\omega}$.

Proof. $1^{\circ}$ is trivial by the definitions.

Since $M^{\omega}$ is a $\mathrm{II}_{1}$ factor $([\mathrm{McD}]),\left(M^{\omega}\right)_{1}$ is complete in the norm \|\|$_{2}$. Thus, the completeness of $(\mathcal{X})_{1}$ follows from the first part of $2^{\circ}$.

To prove the first part of $2^{\circ}$, let $\varepsilon>0$ and $m \geq 1$. Then there exists $k$ such that $\left\|x-x^{k}\right\|_{2}<\varepsilon / 3$. Thus, if $x^{k}=\left(x_{n}^{k}\right)_{n}, x=\left(x_{n}\right)_{n}$, then there exists a neighborhood $V^{\prime}$ of $\omega$ such that $\left\|x_{n}^{k}-x_{n}\right\|_{2} \leq \varepsilon / 3, \forall n \in V^{\prime}$. But since $x^{k} \in \mathcal{X}$, it follows that there exists a neighborhood $V^{\prime \prime}$ of $\omega$ such that $\left\|e_{m}\left(\hat{x}_{n}^{k}\right)-\hat{x}_{n}^{k}\right\|_{2} \leq \varepsilon / 3, \forall n \in V^{\prime \prime}$. Hence, if we denote $V=V^{\prime} \cap V^{\prime \prime}$ then for $n \in V$ we have:

$$
\begin{gathered}
\left\|e_{m}\left(\hat{x}_{n}\right)-\hat{x}_{n}\right\|_{2} \leq\left\|e_{m}\left(\hat{x}_{n}\right)-e_{m}\left(\hat{x}_{n}^{k}\right)\right\|_{2} \\
+\left\|e_{m}\left(\hat{x}_{n}^{k}\right)-\hat{x}_{n}^{k}\right\|_{2}+\left\|\hat{x}_{n}^{k}-\hat{x}_{n}\right\|_{2} \leq 3 \varepsilon / 3=\varepsilon .
\end{gathered}
$$

This shows that $x \in \mathcal{X}$.

$\begin{array}{ll}\text { The proof that } \mathcal{Y} \cap\left(M^{\omega}\right)_{1} \text { is complete is similar. } & \text { Q.E.D. }\end{array}$

1.5. Lemma. $\mathcal{Y}=M^{\omega} \ominus\left(N^{\omega} \rtimes G\right)$. Equivalently, if $x \in M^{\omega}$ then $x \in \mathcal{Y}$ iff $E_{N^{\omega} \rtimes G}(x)=0$. Also, $L(G)^{\omega} \cap \mathcal{Y}=L(G)^{\omega} \ominus L(G)$.

Proof. This is trivial by the definitions.

Q.E.D.

\section{Multiplicative Properties of Clustering SEQUenCES}

Unless otherwise specified, throughout this section $\sigma$ denotes a clustering action of $G$ on $(N, \tau)$ with clustering resolution $\left(N^{0},\left\{B_{n}\right\}_{n}\right)$, as in Definition 1.1. Also, $\mathcal{X}$ denotes its associated set of sequences with clustering coefficients, as in 1.3 '. We prove here some multiplicative properties for $\mathcal{X}$. 
2.1. Lemma. $1^{\circ} \cdot \mathcal{X} \cap N^{\omega}$ is equal to the von Neumann algebra $\cap_{m} B_{m}^{\omega}$. Also, $E_{N^{\omega}}(\mathcal{X}) \subset \mathcal{X}$.

$2^{\circ} . \mathcal{X}$ is a $L(G)-L(G)^{\omega}$ bimodule.

Proof. $1^{\circ}$. We clearly have $\cap_{m} B_{m}^{\omega} \subset \mathcal{X} \cap N^{\omega}$, by the definitions. Conversely, if $x=\left(x_{n}\right)_{n} \in N^{\omega}$ belongs to $\mathcal{X}$ then for any $m \geq 1$ and any $\varepsilon>0$ there exists a neighborhood $V$ of $\omega$ such that $\left\|E_{B_{m}}\left(x_{n}\right)-x_{n}\right\|_{2} \leq \varepsilon, \forall n \in V$. But this implies $\left\|E_{B_{m}^{\omega}}(x)-x\right\|_{2} \leq \varepsilon$ and since $\varepsilon>0$ was arbitrary, this shows that $x \in B_{m}^{\omega}$. Since $m$ was arbitrary as well, $x \in \cap_{m} B_{m}^{\omega}$.

Since for $x=\left(x_{n}\right)_{n} \in M^{\omega}$ we have $E_{N^{\omega}}(x)=\left(E_{N}\left(x_{n}\right)\right)_{n}$, the last part is now clear by the first part and the definitions.

$2^{\circ}$. Let $x=\left(x_{n}\right)_{n} \in \mathcal{X}$ and $y=\left(y_{n}\right)_{n} \in L(G)^{\omega}$, with $y_{n} \in(L(G))_{1}, \forall g$. By the definition 1.3 , for any $m \geq 1$ and $\varepsilon>0$ there exists a neighborhood $V$ of $\omega$ and $\left\{\xi_{n}\right\}_{n} \subset L^{2}\left(\Sigma_{g} B_{m} u_{g}\right)$ such that $\left\|\hat{x}_{n}-\xi_{n}\right\|_{2} \leq \varepsilon, \forall n \in V$. But then $\eta_{n}=\xi_{n} y_{n}$ belongs to $L^{2}\left(\Sigma B_{m} u_{g}\right)$ and $\left\|x_{n} y_{n}-\eta_{n}\right\|_{2} \leq \varepsilon, \forall n \in V$. Thus, $x y \in \mathcal{X}$.

To prove that $\mathcal{X}$ is a left $L(G)$-module, by 1.4 it is sufficient to show that if $x \in \mathcal{X}$ then $u_{g} x \in \mathcal{X}, \forall g \in G$. By Definition 1.3, for any $\varepsilon>0$ and any $m$ there exist $m_{0}, V \in \omega$ and $\xi_{n}^{\prime} \in L^{2}\left(\Sigma_{g} B_{m_{0}} u_{g}\right)$ such that $\sigma_{g}\left(B_{m_{0}}\right) \subset B_{m}$ and $\left\|\hat{x}_{n}-\xi_{n}^{\prime}\right\|_{2} \leq \varepsilon$, $\forall n \in V$. But then we have $u_{g} \xi_{n}^{\prime} \in L^{2}\left(\Sigma_{g} B_{m} u_{g}\right)$. Thus, $\left\|u_{g} \hat{x}_{n}-u_{g} \xi_{n}^{\prime}\right\|_{2} \leq \varepsilon$, $\forall n \in V$. This shows that $\left(u_{g} x_{n}\right)_{n} \in \mathcal{X}$.

Q.E.D.

2.2. Lemma. The unitaries $u_{g}, g \in G$, normalize $\mathcal{X} \cap N^{\omega}=\cap_{m} B_{m}^{\omega}$ and $\mathcal{X} \cap$ $\left(N^{\omega} \rtimes G\right)$ is equal to the von Neumann algebra $\left(\cap_{m} B_{m}^{\omega}\right) \rtimes G$, generated by $\cap_{m} B_{m}^{\omega}$ and $\left\{u_{g}\right\}_{g}$. Also, $E_{N^{\omega} \rtimes G}(\mathcal{X}) \subset \mathcal{X}$.

Proof. By (1.1.3) and Definition 1.3, we clearly have $u_{g}\left(\cap_{m} B_{m}^{\omega}\right) u_{g}^{*}=\cap_{m} B_{m}^{\omega}$. By 2.1.2 $2^{\circ}$, the ${ }^{*}$-algebra generated by $\cap B_{m}^{\omega}$ and $\left\{u_{g}\right\}_{g}$ is contained in $\mathcal{X}$. Conversely, if $x=\left(x_{n}\right)_{n} \in \mathcal{X} \cap\left(N^{\omega} \rtimes G\right)$ then $x=\Sigma_{g} E_{N^{\omega}}\left(x u_{g}^{*}\right) u_{g}$ and by $2.1 .1^{\circ}$ each $E_{N^{\omega}}\left(x u_{g}^{*}\right), g \in G$, follows in $\mathcal{X} \cap N^{\omega}=\cap_{m} B_{m}^{\omega}$. Thus, $x \in\left(\cap_{m} B_{m}^{\omega}\right) \rtimes G$.

To see that if $x \in \mathcal{X}$ then $E_{N^{\omega} \rtimes G}(x) \in \mathcal{X}$, note that $E_{N^{\omega}}\left(x u_{g}^{*}\right) u_{g} \in\left(\cap_{m} B_{m}^{\omega}\right) \rtimes G$, so by taking finite sums and limits we get $E_{N^{\omega} \rtimes G}(x) \in L^{2}\left(\left(\cap_{m} B_{m}^{\omega}\right) \rtimes G\right)$, implying that $E_{N^{\omega} \rtimes G}(x) \in\left(\cap_{m} B_{m}^{\omega}\right) \rtimes G \subset \mathcal{X}$.

Q.E.D.

2.3. Corollary. If $x \in \mathcal{X}$, then $x-E_{N^{\omega} \rtimes G}(x) \in \mathcal{X} \cap \mathcal{Y}$.

Proof. By the last part of 2.2 we have $x-E_{N^{\omega} \rtimes G}(x) \in \mathcal{X}$ while by 1.5 we have $x-E_{N^{\omega} \rtimes G}(x) \in \mathcal{Y}$.

Q.E.D.

2.4. Lemma. $\mathcal{X} \cap \mathcal{Y}$ is a right $M$-module, i.e., if $x \in \mathcal{X} \cap \mathcal{Y}$ then $x M \subset \mathcal{X} \cap \mathcal{Y}$. In particular, $\left(L(G)^{\omega} \ominus L(G)\right) M \subset \mathcal{X}$. 
Proof. By 1.4 it is sufficient to prove that $x y \in \mathcal{X} \cap \mathcal{Y}$ for $y \in N^{0} \cup\left\{u_{g}\right\}_{g}$. The case $y=u_{g}$ is trivial by the definitions. Let $y \in N^{0},\|y\| \leq 1$. Then clearly $x y \in \mathcal{Y}$. To show that $x y \in \mathcal{X}$ as well, let $m \geq 1, \varepsilon>0$. By Definition 1.1 there exists $F \subset G$ finite such that $\sigma_{g}(y) \in B_{m}, \forall g \in G \backslash F$. On the other hand, by 1.3, since $x \in \mathcal{X}$ there exists a neighborhood $V$ of $\omega$ so that $\left\|e_{m}\left(\hat{x}_{n}\right)-\hat{x}_{n}\right\|_{2} \leq \varepsilon / 2, \forall n \in V$. Also, since $x \in \mathcal{Y}, V$ can be chosen so that if $x_{n}=\Sigma_{g} y_{n, g} u_{g}$, with $y_{n, g} \in N$, then $\left\|y_{n, g}\right\|_{2} \leq \varepsilon / 3|F|^{1 / 2}, \forall n \in V$. All this entails the following estimates:

$$
\begin{aligned}
& \left\|e_{m}\left(\hat{x_{n}} y\right)-\hat{x_{n}} y\right\|_{2}^{2}=\Sigma_{g \in G}\left\|E_{B_{m}}\left(y_{n, g} \sigma_{g}(y)\right)-y_{n, g} \sigma_{g}(y)\right\|_{2}^{2} \\
& =\Sigma_{g \in F}\left\|E_{B_{m}}\left(y_{n, g} \sigma_{g}(y)\right)-y_{n, g} \sigma_{g}(y)\right\|_{2}^{2} \\
& +\Sigma_{g \in G \backslash F}\left\|E_{B_{m}}\left(y_{n, g} \sigma_{g}(y)\right)-y_{n, g} \sigma_{g}(y)\right\|_{2}^{2} \\
& \leq 4 \Sigma_{g \in F}\left\|y_{n, g}\right\|_{2}^{2}+\Sigma_{g \in G \backslash F}\left\|\left(E_{B_{m}}\left(y_{n, g}\right)-y_{n, g}\right) \sigma_{g}(y)\right\|_{2}^{2} \\
& <\varepsilon^{2} / 2+\left\|e_{m}\left(\hat{x}_{n}\right)-\hat{x}_{n}\right\|_{2}^{2}<\varepsilon^{2} .
\end{aligned}
$$

Thus $x y \in \mathcal{X}$.

Q.E.D.

While by $2.4 \mathcal{X} \cap \mathcal{Y}$ is invariant to multiplication by $M$ from the right, the next result shows that multiplication from the left by elements in $M \ominus L(G)$ takes the space $\mathcal{X} \cap \mathcal{Y}$ perpendicular to $\mathcal{X}$ :

2.5. Lemma. $(M \ominus L(G))(\mathcal{X} \cap \mathcal{Y}) \perp \mathcal{X}$.

Proof. Let $x=\left(x_{n}\right)_{n} \in \mathcal{X} \cap \mathcal{Y}, y \in M$ with $E_{L(G)}(y)=0$. We have to show that $\lim _{n \rightarrow \omega} \tau\left(z_{n}^{*} y x_{n}\right)=0, \forall z=\left(z_{n}\right)_{n} \in \mathcal{X}$.

By linearity and Kaplansky's density theorem it is clearly sufficient to prove the statement for $y$ of the form $y=y_{g} u_{g}$ for some $g \in G$ and $y_{g} \in N^{0}, \tau\left(y_{g}\right)=0$. Also, since by 2.1 we have $u_{g}(\mathcal{X} \cap \mathcal{Y}) \subset \mathcal{X} \cap \mathcal{Y}$, by replacing $\left(x_{n}\right)_{n}$ by $\left(u_{g} x_{n}\right)_{n}$ it follows that it is in fact sufficient to prove the statement for $y \in N^{0}$ with $\tau(y)=0$. In addition, we may assume $\|y\| \leq 1,\|x\| \leq 1,\|z\| \leq 1$.

Since $e_{m}(\hat{y})=\hat{E_{B_{m}}}(y)$, we have $e_{m}(y \xi)=E_{B_{m}}(y) \xi$ for any $\xi \in L^{2}\left(\Sigma_{g} B_{m} u_{g}\right)$. As $y \in N^{0}$ and $\tau(y)=0$, by (1.1.1) there exists $m$ such that $E_{B_{m}}(y)=0$. Let $\varepsilon>0$. Since $x, z \in \mathcal{X}$, there exists a neighborhood $V$ of $\omega$ so that $\left\|e_{m}\left(\hat{x}_{n}\right)-\hat{x}_{n}\right\|_{2} \leq \varepsilon / 2$ and $\left\|e_{m}\left(\hat{z}_{n}\right)-\hat{z}_{n}\right\|_{2} \leq \varepsilon / 2, \forall n \in V$. But then, for each $n \in V$ we have the estimates:

$$
\begin{gathered}
\left|\tau\left(z_{n}^{*} y x_{n}\right)\right| \leq\left|\left\langle y e_{m}\left(\hat{x}_{n}\right), e_{m}\left(\hat{z}_{n}\right)\right\rangle\right| \\
+\left\|\hat{z}_{n}-e_{m}\left(\hat{z}_{n}\right)\right\|_{2}\left\|y x_{n}\right\|_{2}+\left\|e_{m}\left(\hat{z}_{n}\right)\right\|_{2}\left\|y \hat{x}_{n}-y e_{m}\left(\hat{x}_{n}\right)\right\|_{2} \\
\leq\left|\left\langle y e_{m}\left(\hat{x}_{n}\right), e_{m}\left(\hat{z}_{n}\right)\right\rangle\right|+\varepsilon=\varepsilon
\end{gathered}
$$


where for the last equality we have used

$$
\left\langle y e_{m}\left(\hat{x}_{n}\right), e_{m}\left(\hat{z}_{n}\right)\right\rangle=\left\langle e_{m}\left(y e_{m}\left(\hat{x}_{n}\right)\right), e_{m}\left(\hat{z}_{n}\right)\right\rangle=\left\langle E_{B_{m}}(y) e_{m}\left(\hat{x}_{n}\right), e_{m}\left(\hat{z}_{n}\right)\right\rangle=0 .
$$

Q.E.D.

Since $L(G)^{\omega} \ominus L(G) \subset \mathcal{X} \cap \mathcal{Y}$, Lemmas 2.4, 2.5 imply that $(M \ominus L(G))\left(L(G)^{\omega} \ominus\right.$ $L(G)) \perp\left(L(G)^{\omega} \ominus L(G)\right) M$. This orthogonality holds in fact true in a larger generality:

2.6. Lemma. Let $\sigma$ be a free, mixing action of a discrete group $G$ on a finite von Neumann algebra $(N, \tau)$ and denote $M=A \rtimes_{\sigma} G$. If $\left(x_{n}\right)_{n},\left(y_{n}\right)_{n} \in L(G)$ are bounded sequences that weakly converge to 0 , then $\lim _{n \rightarrow \infty} \tau\left(x_{n}^{*} a^{*} y_{n} b\right)=0$ for all $a, b \in M \ominus L(G)$. Thus, $(M \ominus L(G))\left(L(G)^{\omega} \ominus L(G)\right) \perp\left(L(G)^{\omega} \ominus L(G)\right) M$.

Proof. We may assume $\left\|x_{n}\right\|,\left\|y_{n}\right\| \leq 1, \forall n$. By linearity and Kaplansky's density theorem, it is sufficient to prove the statement for $a=a_{0} u_{g}, b=b_{0} u_{h}$, where $g, h \in G$ and $a_{0}, b_{0} \in N$. Also, since $\left(u_{g} x_{n}\right)_{n},\left(y_{n} u_{h}\right)_{n}$ are still converging weakly to 0 , it follows that it is in fact sufficient to prove the statement for $a, b \in N$ with $\tau(a)=\tau(b)=0,\|a\|,\|b\| \leq 1$. For such $a, b$, if we let $x_{n}=\Sigma_{g} c_{g}^{n} u_{g}, y_{n}=\Sigma_{g} d_{g}^{n} u_{g}$, $c_{g}^{n}, d_{g}^{n} \in \mathbb{C}$, be the Fourier expansions, then $\tau\left(x_{n}^{*} a^{*} y_{n}\right)=\Sigma_{g \in G} c_{g}^{n} \overline{d_{g}^{n}} \tau\left(a^{*} \sigma_{g}(b)\right)$, with the sum being $\ell^{1}(G)$ convergent.

Let $\varepsilon>0$. Since $\sigma$ is mixing and $\tau(a)=0$, there exists a finite subset $F \subset G$ such that $\left|\tau\left(a^{*} \sigma_{g}(b)\right)\right| \leq \varepsilon / 2, \forall g \in G \backslash F$. By the weak convergence of the sequences $\left(x_{n}\right)_{n},\left(y_{n}\right)_{n}$, there exists $n_{0} \geq 1$ such that $\Sigma_{g \in F}\left|c_{g}^{n} \overline{d_{g}^{n}}\right| \leq \varepsilon / 2, \forall n \geq n_{0}$. Altogether, for $n \geq n_{0}$ we get the estimates:

$$
\begin{gathered}
\left|\tau\left(x_{n}^{*} a^{*} y_{n}\right)\right|=\left|\Sigma_{g \in G} c_{g}^{n} \overline{d_{g}^{n}} \tau\left(a^{*} \sigma_{g}(b)\right)\right| \\
\leq \Sigma_{g \in F}\left|c_{g}^{n} \overline{d_{g}^{n}}\right||| a||\|b\|+\Sigma_{g \notin F}\left|c_{g}^{n} \overline{d_{g}^{n}}\right|\left|\tau\left(a^{*} \sigma_{g}(b)\right)\right| \\
\leq \varepsilon / 2+(\varepsilon / 2) \Sigma_{g \notin F}\left|c_{g}^{n} \overline{d_{g}^{n}}\right| \leq \varepsilon .
\end{gathered}
$$

Q.E.D.

\section{Clustering algebras associated to pairs of aCtions}

Let $\sigma: G \rightarrow \operatorname{Aut}(N, \tau)$ be a properly outer clustering action with clustering resolution $\left(N^{0},\left\{B_{n}\right\}_{n}\right)$, as in Sections 1, 2. In this Section we assume that for a non-zero projection $p \in L(G)=\left\{u_{g}\right\}_{g}{ }^{\prime \prime}$ the reduced algebra $M_{0}=p M p$ has another cross-product decomposition $M_{0}=N_{0} \rtimes_{\sigma_{0}} G_{0}$, for some properly outer action $\sigma_{0}: G_{0} \rightarrow \operatorname{Aut}\left(N_{0}, \tau\right)$.

3.1. Notation. We denote $\mathcal{X}_{0}=\mathcal{X} \cap\left(N_{0}^{\prime} \cap N_{0}^{\omega}\right)$. Note that by $1.4 .1^{\circ}, \mathcal{X}_{0}$ is a vector subspace of $p M^{\omega} p$. We show that in fact : 
3.2. Theorem. $\mathcal{X}_{0}$ is a von Neumann subalgebra of $p\left(N^{\omega} \rtimes G\right) p$.

Proof. We first prove that $\forall \varepsilon>0, \exists y_{0} \in \mathcal{U}\left(N_{0}\right)$ such that $\left\|E_{L(G)}\left(y_{0}\right)\right\|_{2} \leq \varepsilon$. Indeed, for if not then $\exists \varepsilon_{0}>0$ such that if we denote by $\left(\left\langle M, e_{L(G)}\right\rangle, T r\right)$ the basic construction algebra corresponding to the inclusion $L(G) \subset M$, then

$$
\operatorname{Tr}\left(e_{L(G)} y e_{L(G)} y^{*}\right)=\left\|E_{L(G)}(y)\right\|_{2}^{2} \geq \varepsilon_{0}^{2}, \forall y \in \mathcal{U}\left(N_{0}\right)
$$

Taking weak limits of convex combinations of elements of the form $y e_{L(G)} y^{*}$, for $y \in \mathcal{U}\left(N_{0}\right)$, it follows that $\operatorname{Tr}\left(e_{L(G)} a\right) \geq \varepsilon_{0}$ for any $a \in K=\overline{\mathrm{Co}}^{w}\left\{y e_{L(G)} y^{*}\right.$ $\left.y \in \mathcal{U}\left(N_{0}\right)\right\}$. In particular, $\operatorname{Tr}\left(e_{L(G)} a_{0}\right) \geq \varepsilon_{0}^{2}$ for $a_{0}$ the unique element of minimal norm $\|\cdot\|_{2, T r}$ in $K$. Thus, $a_{0} \neq 0$. But $y a_{0} y^{*} \in K$ and $\left\|y a_{0} y^{*}\right\|_{2, T r}=\left\|a_{0}\right\|_{2, T r}$, $\forall y \in \mathcal{U}\left(N_{0}\right)$, so by the uniqueness of $a_{0}$ we have $y a_{0} y^{*}=a_{0}, \forall y \in \mathcal{U}\left(N_{0}\right)$, showing that $a_{0} \in N_{0}^{\prime} \cap\left\langle M, e_{L(G)}\right\rangle, 0 \leq a_{0} \leq 1, \operatorname{Tr}\left(a_{0}\right) \leq \operatorname{Tr}\left(e_{L(G)}\right)=1, a_{0} \neq 0$.

By (Theorem 2.1 in [Po4]), this implies there exists a non-zero projection $q \in N_{0}$, a projection $p \in L(G)$, a unital isomorphism $\psi$ of $q N_{0} q$ into $p L(G) p$ and a partial isometry $v \in M$ such that $v v^{*}=q, v^{*} v \in \psi\left(q N_{0} q\right)^{\prime} \cap p L(G) p$ and $x v=v \psi(x)$, $\forall x \in q N_{0} q$. Moreover, by shrinking $q$ if necessary, we may assume the central trace of $q$ in $N_{0}$ is a scalar multiple of a central projection of $N_{0}$ (any projection in $N_{0}$ majorizes a projection with this latter property).

But $\sigma$ is mixing and $\psi\left(q N_{0} q\right)$ is a subalgebra without atoms in $L(G)$, so by (Theorem 3.1 in [Po4]) it follows that $\psi\left(q N_{0} q\right)^{\prime} \cap p L(G) p \subset L(G)$. Thus, $p_{0}=$ $v^{*} v \in L(G)$ and $v^{*} N_{0} v \subset p_{0} L(G) p_{0}$. Moreover, by applying again (3.1 in [Po4]) it follows that the normalizer of $v^{*} N_{0} v$ in $p_{0} M p_{0}$ is included in $p_{0} L(G) p_{0}$. This is a contradiction, since by $\left(3.5 .2^{\circ}\right.$ in [Po4]) the normalizer of $v^{*} N_{0} v$ in $p_{0} M p_{0}$ generates all $p_{0} M p_{0}$ (because $q N_{0} q$ is regular in $q M q$, due to the fact that $N_{0}$ is regular in $M)$.

Let now $x=\left(x_{n}\right)_{n} \in \mathcal{X}_{0} \subset \mathcal{X}$ and denote $x^{\prime}=x-E_{N^{\omega} \rtimes G}(x)$. By Lemma 2.3, $x^{\prime}$ lies in $\mathcal{X} \cap \mathcal{Y}$. Let $\varepsilon>0$ and choose $y_{0} \in \mathcal{U}\left(N_{0}\right)$ such that $\left\|E_{L(G)}\left(y_{0}\right)\right\|_{2} \leq \varepsilon$. Since $\left[y_{0}, x\right]=0$ and $y_{0} \in M \subset N^{\omega} \rtimes G$, it follows that $\left[y_{0}, E_{N^{\omega} \rtimes G}(x)\right]=0$. Thus, $\left[y_{0}, x^{\prime}\right]=0$.

But by Lemma 2.4 we have $x^{\prime} y_{0} \in \mathcal{X}$, while by Lemma 2.5 we have $\left(y_{0}-\right.$ $\left.E_{L(G)}\left(y_{0}\right)\right) x^{\prime} \perp \mathcal{X}$. In particular, $\left(y_{0}-E_{L(G)}\left(y_{0}\right)\right) x^{\prime}$ is perpendicular to $x^{\prime} y_{0}$. Thus, the commutation relation $y_{0} x^{\prime}=x^{\prime} y_{0}$ entails the equation

$$
x^{\prime} y_{0}-\left(y_{0}-E_{L(G)}\left(y_{0}\right)\right) x^{\prime}=E_{L(G)}\left(y_{0}\right) x^{\prime},
$$

with the vectors $x^{\prime} y_{0},\left(y_{0}-E_{L(G)}\left(y_{0}\right)\right) x^{\prime}$ perpendicular. By Pythagora's Theorem, and using that $y_{0}$ is a unitary element, we get 


$$
\left\|x^{\prime}\right\|_{2}=\left\|x^{\prime} y_{0}\right\|_{2} \leq\left\|E_{L(G)}\left(y_{0}\right) x^{\prime}\right\|_{2} \leq \varepsilon .
$$

Since $\varepsilon>0$ was arbitrary, $x^{\prime}=0$. Thus $x=E_{N^{\omega} \rtimes G}(x) \in N^{\omega} \rtimes G$. But this also shows that $\mathcal{X}_{0}=\left(\mathcal{X} \cap\left(N^{\omega} \rtimes G\right)\right) \cap\left(N_{0}^{\prime} \cap N_{0}^{\omega}\right)$. Since by Lemma $2.2 \mathcal{X} \cap\left(N^{\omega} \rtimes G\right)$ is a weakly closed ${ }^{*}$-algebra, this shows that $\mathcal{X}_{0}$ is a von Neumann algebra as well. Q.E.D.

The next Lemma and its Corollary will be needed in Section 4 .

3.3. Lemma. Given any $\varepsilon>0$ there exists a finite subset $F \subset G$ such that if $p_{F}$ denotes the orthogonal projection of $L^{2}\left(M^{\omega}\right)$ onto $L^{2}\left(\Sigma_{g \in F} N^{\omega} u_{g}\right)$ then for any $x \in\left(\mathcal{X}_{0}\right)_{1}$ we have $\left\|\hat{x}-p_{F}(\hat{x})\right\|_{2} \leq \varepsilon$.

Proof. Assume this is not the case. If $\left\{y_{j}\right\}_{j}$ is a $\|\quad\|_{2}$-dense sequence in $\left(N_{0}\right)_{1}$ and $F_{n}$ is an increasing sequence of finite subsets of $G$ with $\cup_{n} F_{n}=G$, then by the contradiction assumption and the definition of $\mathcal{X}_{0}$, there exists $\varepsilon_{0}>0$ such that for each $n$ there exists $x_{n} \in\left(N_{0}\right)_{1}$ with $\left\|\left[y_{j}, x_{n}\right]\right\|_{2} \leq 1 / n, \forall j \leq n,\left\|\hat{x}_{n}-p_{F_{n}}\left(\hat{x}_{n}\right)\right\|_{2} \geq \varepsilon_{0}$ and $\left\|e_{n}\left(\hat{x}_{n}\right)-x_{n}\right\|_{2} \leq 1 / n$. (Like in Section 1 , if $m \geq 1$ then $e_{m}$ denotes the orthogonal projection of $L^{2}(M)$ onto $L^{2}\left(\Sigma_{g \in G} B_{m} u_{g}\right)$.)

But then $x=\left(x_{n}\right)_{n} \in\left(N_{0}^{\omega}\right)_{1}$ satisfies $\left[x, N_{0}\right]=0, x \in \mathcal{X}$ and $\left\|x-E_{N^{\omega} \rtimes G}(x)\right\|_{2} \geq$ $\varepsilon_{0}$. Since the first two conditions imply $x \in \mathcal{X}_{0}$, the third condition contradicts Theorem 3.2.

Q.E.D.

3.4. Corollary. $\left(\mathcal{X}_{0}^{\prime} \cap\left\langle M^{\omega}, N^{\omega}\right\rangle\right)_{+}$contains non-zero finite projections.

Proof. By Lemma 3.3, there exists $F \subset G$ finite such that $\left\|p_{F}(\hat{v})\right\|_{2} \geq 1 / 2, \forall v \in$ $\mathcal{U}\left(\mathcal{X}_{0}\right)$. Note that $p_{F}=\Sigma_{g \in F} u_{g} e_{N \omega} u_{g}^{*} \in\left\langle M^{\omega}, e_{N \omega}\right\rangle$. Thus, if \|\|$_{2, T r}$ denotes the canonical trace on $\left\langle M^{\omega}, e_{N^{\omega}}\right\rangle$ (see for instance Sec. 2 in [Po4]) then we have:

$$
\begin{gathered}
\operatorname{Tr}\left(p_{F} v p_{F} v^{*}\right)=\Sigma_{h, k \in F}\left\|E_{N^{\omega}}\left(u_{h}^{*} v u_{k}\right)\right\|_{2}^{2} \\
=\Sigma_{h, k \in F}\left\|E_{N^{\omega}}\left(v u_{k h^{-1}}\right)\right\|_{2}^{2} \geq\left\|p_{F}(v)\right\|_{2}^{2} \geq 1 / 4 .
\end{gathered}
$$

Thus, if we take $a \in\left\langle M^{\omega}, e_{N \omega}\right\rangle$ to be the unique element of minimal norm $\|\quad\|_{2, T r}$ in $\overline{\operatorname{co}}\left\{v p_{F} v^{*} \mid v \in \mathcal{U}\left(\mathcal{X}_{0}\right)\right\}$ then $0 \leq a \leq 1, \operatorname{Tr}(a) \leq|F|<\infty$ and $\operatorname{Tr}\left(p_{F} a\right) \geq 1 / 4$. This implies $a \neq 0$ while by uniqueness we have $v a v^{*}=a$, $\forall v \in \mathcal{U}\left(\mathcal{X}_{0}\right)$. Thus, any spectral projection corresponding to an interval $[c, 1]$ with $c>0$ small enough will do.

Q.E.D.

The most important technical result of this section shows that if in addition to the general assumptions set forth at the beginning of this section (i.e. $\sigma$ clustering, $\left.p M p=M_{0}, p L(G) p \supset L\left(G_{0}\right)\right)$ we also assume $p L(G) p \supset L\left(G_{0}\right)$ and $N_{0}$ abelian, then the von Neumann algebra $\mathcal{X}_{0}$ is "large" in $M^{\omega}$. More generally we have: 
3.5. Theorem. If $N_{0}$ is abelian and there exists an infinite subgroup $G_{0}^{\prime} \subset G_{0}$ such that $p L(G) p \supset L\left(G_{0}^{\prime}\right)$ then $\mathcal{X}_{0}^{\prime} \cap p M^{\omega} p=N_{0}^{\omega}$.

Proof. Assume that $x=\left(x_{n}\right)_{n} \in \mathcal{X}_{0}^{\prime} \cap p M^{\omega} p$ but $x \notin N_{0}^{\omega}$. Since $N_{0}$ is abelian, $N_{0}^{\prime} \cap N_{0}^{\omega}=N_{0}^{\omega}$ so $\mathcal{X}_{0}=\mathcal{X} \cap N_{0}^{\omega}$ is a von Neumann subalgebra of $N_{0}^{\omega}$ (cf. 3.2) and $N_{0}^{\omega} \subset \mathcal{X}_{0}^{\prime} \cap p M^{\omega} p$.

Thus, by replacing $x$ by $x-E_{N_{0}^{\omega}}(x)$, we may assume $x \perp N_{0}^{\omega}$. We may further take $x_{n}=\Sigma_{g} y_{n, g} u_{g}^{0}$ with $y_{n, g} \in N_{0}, y_{n, e}=0, \forall n$. Moreover, by Kaplansky's density theorem we may assume $y_{n, g}=0$ for all $g \in G_{0} \backslash F_{n}$, for some finite subsets $F_{n} \subset G_{0}$.

At this point we need the following immediate consequences of the results in Section 2:

3.6. Lemma. If $\sigma$ is clustering and $N_{0}$ is abelian, then for any sequence $\left\{h_{n}\right\}_{n} \subset$ $G_{0}^{\prime}$ that tends to infinity in $G_{0}^{\prime}$ and any $y_{0} \in N_{0}$, we have $\tilde{y}_{0}=\left(\sigma_{0}\left(h_{n}\right)\left(y_{0}\right)\right)_{n} \in \mathcal{X}_{0}$.

Proof. The sequence $\left(u_{h_{n}}^{0}\right)_{n}$ lies in $L(G)$ and tends weakly to 0 , so $U=\left(u_{h_{n}}^{0}\right)_{n} \in$ $L(G)^{\omega} \ominus L(G)$. By Lemma 2.4 we get $U y_{0} \in \mathcal{X}$ and then by $2.1 .2^{\circ}$ we get $\left(U y_{0}\right) U^{*} \in$ $\mathcal{X}$. Since $N_{0}$ is abelian, it follows that $U y_{0} U^{*} \in N_{0}^{\prime} \cap N_{0}^{\omega}=N_{0}^{\omega}$ as well. Thus $\tilde{y}_{0}=U y_{0} U^{*} \in \mathcal{X}_{0}$.

Q.E.D.

3.7. Lemma. If $\sigma$ is clustering and $N_{0}$ is abelian, then $\sigma_{0 \mid G_{0}^{\prime}}$ is $n$-mixing, $\forall n \geq 1$.

Proof. It is sufficient to prove that if $y_{0}, y_{1}, \ldots, y_{n} \in N_{0}$ and $\left\{h_{n}^{i}\right\}_{n} \subset G_{0}^{\prime}$ are sequences tending to $\infty, 1 \leq i \leq n$, then $\lim _{n \rightarrow \infty} \tau\left(y_{0} \Pi_{1 \leq i \leq n} \sigma_{0}\left(h_{n}^{i}\right)\left(y_{i}\right)\right)=0$. This amounts to showing that $U_{i}=\left(u_{h_{n}^{i}}^{0}\right)_{n} \in M^{\omega}$ satisfy $\tau\left(y_{0} Y\right)=\tau\left(y_{0}\right) \tau(Y)$, where $Y=\Pi_{1 \leq i \leq n} U_{i} y_{i} U_{i}^{*}$.

As in the proof of 3.6, $h_{n}^{i} \rightarrow \infty$ implies $U_{i} \in L(G)^{\omega} \ominus L(G)$. Applying 3.6 and 3.2 , we get $Y \in \mathcal{X}_{0}$. But $E_{M}(\mathcal{X}) \subset \mathbb{C} 1$, thus $E_{M}(Y)=\tau(Y) 1$ and finally

$$
\tau\left(y_{0} Y\right)=\tau\left(E_{M}\left(y_{0} Y\right)\right)=\tau\left(y_{0} E_{M}(Y)\right)=\tau\left(y_{0}\right) \tau(Y) .
$$

Q.E.D.

Proof of 3.5 (continuation). Since $\sigma_{0}$ satisfies condition (1.2.1) for $n=2$, by applying 1.3 and (1.2.1) to sequences of the form $\left(\sigma_{0}\left(h_{n}\right)\left(y_{0}\right)\right)_{n}$ for $y_{0} \in N_{0}$, $h_{n} \in G_{0}^{\prime}, h_{n} \rightarrow \infty$ (which by Lemma 3.6 belong to $\mathcal{X}_{0}$ ), it follows that for any finite sets $Y \subset N_{0}, F \subset G_{0}$, any $m, k \geq 1$ and any $\varepsilon>0$ there exists $n>k$ such that

$$
\left|\tau\left(y_{0} \sigma_{0}\left(h_{n}\right)\left(y_{1}\right) \sigma_{0}\left(g h_{n}\right)\left(y_{2}\right)\right)-\tau\left(y_{0}\right) \tau\left(\sigma_{0}\left(h_{n}\right)\left(y_{1}\right) \sigma_{0}\left(g h_{n}\right)\left(y_{2}\right)\right)\right| \leq \varepsilon
$$


for any $y_{0}, y_{1}, y_{2} \in Y, g \in F$.

Let $q \in \mathcal{P}\left(N_{0}\right)$ with $\tau(q)=1 / 2$ and let $F^{\prime} \subset G_{0}^{\prime}$ finite such that $\tau\left(q \sigma_{0}\left(g^{\prime}\right)(q)\right) \leq$ $9 / 32$ for $g^{\prime} \in G_{0}^{\prime} \backslash F^{\prime}$ (this is possible because $\sigma_{0 G_{0}^{\prime}}$ is 2-mixing, hence mixing). Thus, $\tau\left(q \sigma_{0}\left(g^{\prime}\right)(q)\right)+\tau\left((1-q) \sigma_{0}\left(g^{\prime}\right)(1-q)\right) \leq 9 / 16$. Since $\sigma_{0}$ is a free action, there exists a finite partition $\left\{q_{i}\right\}_{i} \subset \mathcal{P}\left(N_{0}\right)$ refining $\{q, 1-q\}$ such that $\Sigma_{i} \tau\left(q_{i} \sigma_{0}\left(g^{\prime}\right)\left(q_{i}\right)\right) \leq 9 / 16$, for all $g^{\prime} \in F^{\prime}, g^{\prime} \neq e$. Thus, since

$$
\begin{aligned}
& \Sigma_{i}\left\|q_{i} \sigma_{0}\left(g^{\prime}\right)\left(q_{i}\right)\right\|_{2}^{2}=\Sigma_{i} \tau\left(q_{i} \sigma_{0}\left(g^{\prime}\right)\left(q_{i}\right)\right) \\
\leq & \tau\left(q \sigma_{0}\left(g^{\prime}\right)(q)\right)+\tau\left((1-q) \sigma_{0}\left(g^{\prime}\right)(1-q)\right) \\
= & \left\|q \sigma_{0}\left(g^{\prime}\right)(q)\right\|_{2}^{2}+\left\|(1-q) \sigma_{0}\left(g^{\prime}\right)(1-q)\right\|_{2}^{2},
\end{aligned}
$$

altogether we get

$$
\Sigma_{i}\left\|q_{i} \sigma_{0}\left(g^{\prime}\right)\left(q_{i}\right)\right\|_{2}^{2} \leq 9 / 16, \forall g^{\prime} \in G_{0} \backslash\{e\}
$$

Assume $k_{1}<k_{2}<\ldots<k_{n-1}$ are given. By applying (3.5.1) for $\varepsilon=2^{-4}\left\|q_{i}\right\|_{2}^{2}\left\|y_{n, g}\right\|_{2}^{2}$, it follows that there exists $k_{n}>k_{n-1}$ large enough such that for all $g \in F_{n}$ and all $y_{n, g} \neq 0, g \in F_{n}$, we have:

$$
\begin{gathered}
\left\|\left(\sigma_{0}\left(h_{k_{n}}\right)\left(q_{i}\right) \sigma_{0}\left(g h_{k_{n}}\right)\left(q_{i}\right)\right) y_{n, g}\right\|_{2}^{2} \\
\leq\left(\left\|\sigma_{0}\left(h_{k_{n}}\right)\left(q_{i}\right) \sigma_{0}\left(g h_{k_{n}}\right)\left(q_{i}\right)\right\|_{2}^{2}+2^{-4}\left\|q_{i}\right\|_{2}^{2}\right)\left\|y_{n, g}\right\|_{2}^{2}
\end{gathered}
$$

Since the supports $F_{n}$ of the elements $x_{n}$ do not contain $e$ and since $g \in F_{n}$ implies $h g h^{-1} \neq e$ for all $h \in G_{0}$, by applying first (3.5.3) and then (3.5.2) we have the estimates:

$$
\begin{gathered}
\left\|x_{n}\right\|_{2}^{2}=\left\|\Sigma_{i}\left(\sigma_{0}\left(h_{k_{n}}\right)\left(q_{i}\right) x_{n} \sigma_{0}\left(h_{k_{n}}\right)\left(q_{i}\right)\right)\right\|_{2}^{2} \\
=\Sigma_{i} \Sigma_{g \in F_{n}}\left\|\sigma_{0}\left(h_{k_{n}}\right)\left(q_{i}\right) y_{n, g} u_{g}^{0} \sigma_{0}\left(h_{k_{n}}\right)\left(q_{i}\right)\right\|_{2}^{2} \\
=\Sigma_{i} \Sigma_{g \in F_{n}}\left\|\left(\sigma_{0}\left(h_{k_{n}}\right)\left(q_{i}\right) \sigma_{0}\left(g h_{k_{n}}\right)\left(q_{i}\right)\right) y_{n, g}\right\|_{2}^{2} \\
\leq \Sigma_{i} \Sigma_{g \in F_{n}}\left(\left\|\sigma_{0}\left(h_{k_{n}}\right)\left(q_{i}\right) \sigma_{0}\left(g h_{k_{n}}\right)\left(q_{i}\right)\right\|_{2}^{2}+2^{-4}\right)\left\|y_{n, g}\right\|_{2}^{2} \\
=\Sigma_{g \in F_{n}}\left(\Sigma_{i}\left\|q_{i} \sigma_{0}\left(h_{k_{n}}^{-1} g h_{k_{n}}\right)\left(q_{i}\right)\right\|_{2}^{2}+2^{-4}\left\|q_{i}\right\|_{2}^{2}\right)\left\|y_{n, g}\right\|_{2}^{2} \\
\leq(10 / 16) \Sigma_{g \in F_{n}}\left\|y_{n, g}\right\|_{2}^{2}=(5 / 8)\left\|x_{n}\right\|_{2}^{2},
\end{gathered}
$$

a contradiction. 
3.8. Remark. Note that in the above we have actually proved the following more general result: Let $\sigma_{0}$ be a free action of an infinite discrete group $G_{0}$ on a finite von Neumann algebra $\left(N_{0}, \tau_{0}\right)$ and denote $M_{0}=N_{0} \rtimes_{\sigma_{0}} G_{0}$. Assume $\sigma_{0}$ is weakly 2-mixing, i.e. $\forall F \subset N_{0}$ finite $\exists h_{n} \rightarrow \infty$ in $G_{0}$ such that

$\lim _{n, m \rightarrow \infty}\left|\tau\left(y_{0} \sigma_{0}\left(h_{n}\right)\left(y_{1}\right) \sigma_{0}\left(h_{m}\right)\left(y_{2}\right)\right)-\tau\left(y_{0}\right) \tau\left(\sigma_{0}\left(h_{n}\right)\left(y_{1}\right) \sigma_{0}\left(h_{m}\right)\left(y_{2}\right)\right)\right|=0, \forall y_{i} \in F$

Let $x \in M_{0}^{\omega}$ be so that $E_{N_{0}^{\omega}}(x)=0$. Then given any $\varepsilon>0$ there exists a partition $\left\{q_{i}\right\}_{i}$ of 1 with projections in $N_{0}$ and a sequence $h_{n} \rightarrow \infty$ in $G_{0}$ such that the partition $\tilde{q}_{i}=\left(\sigma_{0}\left(h_{n}\right)\left(q_{i}\right)\right)_{n} \in \mathcal{P}\left(N_{0}^{\omega}\right)$ satisfies $\left\|\Sigma_{i} \tilde{q}_{i} x \tilde{q}_{i}\right\|_{2} \leq \varepsilon$.

\section{Cartan conjugacy CRiteria}

We now use Section 3 to prove a criterion for unitary (or inner) conjugacy of Cartan subalgebras. It shows that if $M$ can be realized in two ways as a group measure space construction, $M=L^{\infty}(X, \mu) \rtimes_{\sigma} G=L^{\infty}\left(X_{0}, \mu_{0}\right) \rtimes_{\sigma_{0}} G_{0}$, with $\sigma$ clustering, then the unitary conjugacy of the group von Neumann algebras $L(G), L\left(G_{0}\right)$ entails the unitary conjugacy of the Cartan subalgebras $L^{\infty}(X, \mu), L^{\infty}\left(X_{0}, \mu_{0}\right)$. In fact, in its most general form (see 4.2), this statement assumes only a "corner" of $M$ to have a second group measure space decomposition $A_{0} \rtimes_{\sigma_{0}} G_{0}$, with merely $L\left(G_{0}^{\prime}\right) \subset p L(G) p$ for some infinite subgroup $G_{0}^{\prime} \subset G_{0}$.

Combined with the result in ([Po4]), showing that if $G$ is ICC, $G_{0}$ is w-rigid and $\sigma$ is malleable then the unitary conjugacy of $L\left(G_{0}\right)$ into $L(G)$ holds true automatically, this result already allows us to show that modulo perturbation by inner automorphisms any $\theta: M_{0} \simeq M$ must take the Cartan decompositions $L^{\infty}\left(X_{0}, \mu_{0}\right) \subset M_{0}$, $L^{\infty}(X, \mu) \subset M$ onto each other.

We fix the following general assumptions/notations, throughout the rest of the paper:

4.1. Notations. Let $\sigma: G \rightarrow \operatorname{Aut}(A, \tau), \sigma_{0}: G_{0} \rightarrow \operatorname{Aut}\left(A_{0}, \tau\right)$ be free, ergodic actions of countable discrete groups $G, G_{0}$ on abelian von Neumann algebra $(A, \tau),\left(A_{0}, \tau\right)$. Denote $M=A \rtimes_{\sigma} G, M_{0}=A_{0} \rtimes_{\sigma_{0}} G_{0}$ and $L(G) \subset M$ (respectively $\left.L\left(G_{0}\right) \subset M_{0}\right)$ the von Neumann algebra generated by the canonical unitaries $\left\{u_{g}\right\}_{g \in G} \subset M$ (resp. $\left\{u_{h}^{0}\right\}_{h \in G_{0}} \subset M_{0}$ ) implementing the action $\sigma$ (resp. $\sigma_{0}$ ).

4.2. Theorem. With the notations 4.1, assume that $\sigma$ is clustering and there exists a projection $p \in L(G)$ such that $p M p=M_{0}, p L(G) p \supset L\left(G_{0}^{\prime}\right)$, for some infinite subgroup $G_{0}^{\prime} \subset G_{0}$. Then there exists a partial isometry $v \in M$ such that $v^{*} v=p, v v^{*} \in A$ and $v A_{0} v^{*}=A v v^{*}$. 
Proof. Let $\mathcal{X}_{0} \subset p M^{\omega} p$ be defined as in Section 3. By its definition, $\mathcal{X}_{0}$ is contained in $A_{0}^{\omega}$ and by Theorem $3.2, \mathcal{X}_{0}$ is a von Neumann algebra. Also, by Theorem 3.5 we have $\mathcal{X}_{0}^{\prime} \cap p M^{\omega} p=A_{0}^{\omega}$.

Due to Corollary 3.4, we are in the position of applying the "intertwining theorem" (A.1 in [Po2]). It follows that there exist non-zero projections $q=\left(q_{n}\right)_{n} \in A^{\omega}$, $p^{0}=\left(p_{n}^{0}\right)_{n} \in A_{0}^{\omega} \subset p M^{\omega} p$ and a partial isometry $w=\left(w_{n}\right)_{n} \in M^{\omega}$ such that $q_{n} \in A, p_{n}^{0} \in A_{0}, w_{n}^{*} w_{n}=p_{n}^{0}, w_{n} w_{n}^{*}=q_{n}, \tau\left(q_{n}\right)=\tau\left(p_{n}^{0}\right)=\tau(q), \forall n$, and $w A_{0}^{\omega} w^{*}=A^{\omega} q$. This implies that for $\varepsilon=\tau(q) / 2$ and large enough $n$ we have $w_{n} A_{0} w_{n}^{*} \subset_{\varepsilon} A p_{n}^{0}$. Indeed, because if for each $n$ we could find $u_{n} \in \mathcal{U}\left(w_{n} A_{0} w_{n}^{*}\right)$ such that $\left\|E_{A^{\omega}}\left(u_{n}\right)-u_{n}\right\|_{2} \geq \tau(q) / 2$, then $u=\left(u_{n}\right)_{n}$ would satisfy $w u w^{*} \notin A^{\omega}$, contradicting $w A_{0}^{\omega} w^{*} \subset A^{\omega}$.

By taking $\tilde{A}_{0} \subset M$ to be a Cartan subalgebra of $M$ such that $p \in \tilde{A}_{0}$ and $A_{0}=\tilde{A}_{0} p$, and applying (Corollary 1 in [Po5]) to the Cartan subalgebras $A, \tilde{A}_{0}$ of $M$, it follows that there exists a partial isometry $v \in M$ with $v^{*} v=p$ and $v A_{0} v^{*}=A v v^{*}$.

Q.E.D.

4.3. Remarks. If instead of (Corollary 1 in $[\mathrm{Po} 5])$ we use results from ([PoSiSm $]$ ), or some of Christensen's pioneering results on perturbations of subalgebras in $\mathrm{II}_{1}$ factors $([\mathrm{Ch}])$, then the argument at the end of the proof of 4.2 shows the following: If $A, A_{0}$ are arbitrary maximal abelian ${ }^{*}$-subalgebras in a $\mathrm{II}_{1}$ factor $M$ such that $A_{0}^{\omega}, A^{\omega}$ are unitary conjugate in $M^{\omega}$ then $A_{0}, A$ are unitary conjugate in $M$.

In the next Section, we will prove that if in addition to the conditions in 4.2 we also assume $G$ ICC, then the situation is in fact much more rigid: $p$ must equal 1 and $v$, which follows a unitary, can be taken to satisfy $v\left\{u_{h}^{0}\right\}_{h} v^{*}=\left\{u_{g}\right\}_{g}$, modulo the scalars ! In particular, this will show that the inclusion $L\left(G_{0}\right) \subset p L(G) p$ must be an equality. We prove here a weaker result in this direction, showing that $A_{0}, p L(G) p$ follow mutually orthogonal (i.e., $E_{L(G)}\left(a_{0}\right)=0, \forall a_{0} \in A_{0}, \tau\left(a_{0}\right)=0$; see $[\mathrm{Po} 7])$. This is needed in the proof of the criteria for conjugacy of actions in the next section $(5.1,5.2)$.

4.4. Lemma. With the notations 4.1, assume that for some projection $p \in L(G)$ we have $p M p \supset M_{0}, p L(G) p \supset L\left(G_{0}\right)$ and there exists a partial isometry $v \in M$ such that $v^{*} v=p, v A_{0} v^{*}=A v v^{*}$.

(i). If $\sigma_{0}$ is weakly mixing then $A_{0}$ follows orthogonal to $L(G)$.

(ii). If $\sigma_{0}$ is weakly mixing and $\sigma$ is mixing, then $\sigma_{0}$ follows mixing.

Proof. $(i)$. Note first that $\sigma_{0}$ weakly mixing implies that $\forall y \in A_{0}$ with $\tau(y)=0$ there exist $h_{n} \in G_{0}$ such that $\sigma_{0}\left(h_{n}\right)(y)$ tends to 0 in the weak operator topology, as $n \rightarrow \infty$. 
Assume there exists $y_{0} \in A_{0} \subset p M p$ such that $\tau\left(y_{0}\right)=0, x_{0}=E_{L(G)}\left(y_{0}\right) \neq 0$. Let $h_{n} \in G_{0}$ be so that $u_{h_{n}}^{0} y_{0} u_{h_{n}}^{0 *}=\sigma_{0}\left(h_{n}\right)\left(y_{0}\right)$ tends weakly to 0 . Expecting on $L(G)$ and taking into account that

$$
E_{L(G)}\left(u_{h_{n}}^{0} y_{0} u_{h_{n}}^{0 *}\right)=u_{h_{n}}^{0} E_{L(G)}\left(y_{0}\right) u_{h_{n}}^{0 *}=u_{h_{n}}^{0} x_{0} u_{h_{n}}^{0 *},
$$

it follows that the sequence $\tilde{x}_{0}=\left(u_{h_{n}}^{0} x_{0} u_{h_{n}}^{0 *}\right)_{n} \subset L(G)$ is convergent to 0 in the weak operator topology. Thus, as an element in the ultrapower algebra $L(G)^{\omega}, \tilde{x}_{0}$ belongs to $L(G)^{\omega} \ominus L(G)$, while $\left\|\tilde{x}_{0}\right\|_{2}=\left\|x_{0}\right\|_{2} \neq 0$.

But $A_{0}=v^{*} A v$ implies $A_{0}^{\omega}=v^{*} A^{\omega} v$, and thus $A_{0}^{\omega}$ is contained in $A^{\omega} \rtimes G$, the von Neumann algebra generated by $A^{\omega}$ and the unitaries $u_{g} \in M, g \in G$, regarded as constant sequences in $M^{\omega}$. Thus, if we denote $\tilde{y}_{0}=\left(u_{n}^{0} y_{0} u_{n}^{0 *}\right)_{n} \in A_{0}^{\omega}$ then $\tilde{y}_{0} \in A^{\omega} \rtimes G$. By commuting squares it follows that $E_{L(G) \omega}\left(A^{\omega} \rtimes_{\sigma} G\right)=$ $L(G)^{\omega} \cap A^{\omega} \rtimes_{\sigma} G \subset L(G)$. Thus $E_{L(G)^{\omega}}\left(\tilde{y}_{0}\right) \in L(G)$. On the other hand

$$
E_{L(G)^{\omega}}\left(\tilde{y}_{0}\right)=\left(E_{L(G)}\left(u_{n}^{0} y_{0} u_{n}^{0 *}\right)\right)_{n}=\tilde{x}_{0} \in L(G)^{\omega} \ominus L(G)
$$

This contradiction ends the proof of part $(i)$.

(ii). Let $h_{n} \rightarrow \infty$ in $G_{0}$ and $a_{i} \in A_{0}$ with $\tau\left(a_{i}\right)=0, i=1,2$. Since by $(i)$ we have $A_{0} \perp L(G)$, it follows that $\tau\left(a_{2}\right)=0$ implies $a_{2} \perp L(G)$. But then by 2.6 we get $\lim _{n \rightarrow \infty} \tau\left(a_{2} u_{h_{n}}^{0} a_{1} u_{h_{n}}^{0 *}\right)=0$, showing that $\sigma$ is mixing.

Q.E.D.

Our last result in this section shows that under the same assumptions as in 4.5, the condition $\sigma_{0}$ mixing is in fact not much of a restriction:

4.5. Lemma. With the notations 4.1, assume that for some projection $p \in L(G)$ we have $p M p \supset M_{0}, p L(G) p \supset L\left(G_{0}\right)$. Assume also that either $p M p=M_{0}$ or that there exists $v \in M$ partial isometry such that $v^{*} v=p, v A_{0} v^{*}=A v v^{*}$. If $\sigma$ is mixing then there exist a normal subgroup $G_{1} \subset G_{0}$ of finite index $n$ and a projection $p_{1} \in \mathcal{P}\left(A_{0}\right)$ of trace $\tau\left(p_{1}\right)=\tau(p) / n$ that commutes with $\left\{u_{h}^{0}\right\}_{h \in G_{1}}$, such that if $A_{1} \stackrel{\text { def }}{=} A_{0} p_{1}, \sigma_{1}(h) \stackrel{\text { def }}{=} \sigma_{0}(h)_{\mid A_{1}}, u_{h}^{1} \stackrel{\text { def }}{=} u_{h}^{0} p_{1}, h \in G_{1}$, then:

(i). $\sigma_{1}$ is free and mixing on $A_{1}$ and $M_{1} \stackrel{\text { def }}{=} p_{1} M_{0} p_{1}=A_{1} \vee\left\{u_{h}^{1}\right\}_{h \in G_{1}}{ }^{\prime \prime}$ is isomorphic to $A_{1} \rtimes_{\sigma_{1}} G_{1}$, with $\left\{u_{h}^{1}\right\}_{h}$ as canonical unitaries implementing $\sigma_{1}$.

(ii). $p_{1} M p_{1} \supset M_{1}$, with equality iff $p M p=M_{0} ; p_{1} L(G) p_{1} \supset L\left(G_{1}\right)=\left\{u_{h}^{1}\right\}_{h \in G_{1}}{ }^{\prime \prime}$, with equality iff $p L(G) p=L\left(G_{0}\right)$. Moreover, if $v \in M$ satisfies $v^{*} v=p, v A_{0} v^{*}=$ Avv $v^{*}$ then $v_{1}=v p_{1} \in M$ is a partial isometry satisfying $v_{1}^{*} v_{1}=p_{1}, v_{1} A_{1} v_{1}^{*}=$ $A v_{1} v_{1}^{*}$.

Proof. If $B_{0} \subset A_{0}$ is a finite dimensional $\sigma_{0}$-invariant subalgebra of $A_{0}$ then $\operatorname{Sp} L\left(G_{0}\right) B_{0}=\operatorname{Sp} B_{0} L\left(G_{0}\right)$. Thus, (3.1 in [Po4]) applied to $Q_{0}=L\left(G_{0}\right) p \oplus(1-$ 
p) $L(G)(1-p) \subset A \rtimes_{\sigma} G$ implies that $B_{0}$ is contained in $L(G)$ and therefore in $p L(G) p$. This shows that the von Neumann subalgebra $B \subset A_{0}$ generated by all such $B_{0} \subset A_{0}$ is contained in $p L(G) p$ as well. If $B$ would be infinite dimensional then by the ergodicity of $\sigma$ it follows diffuse. Since the normalizer of $B$ in $p M p$ contains both $A_{0}$ and $\left\{u_{h}^{0} \mid h \in G_{0}\right\}$, which together generate $M_{0}$, by applying again (3.1 in [Po4]) it follows that $M_{0} \subset p L(G) p$. This gives an obvious contradiction when we assume $p M p=M_{0}$. If we assume instead that $A_{0}$ is conjugate to a corner of $A$ by some partial isometry $v \in M$, then $A_{0}$ would contain an orthonormal family of unitary elements that are perpendicular to $A$ (the powers of any generating Haar unitary for $B$ would do), contradicting (A.1 in [Po2], or 2.1 in [Po4]).

Thus $B$ must be finite dimensional and by the ergodicity of $\sigma_{0}$ all its minimal projections have the same trace. Let $n=\operatorname{dim}(B)$ and choose $p_{1} \in \mathcal{P}(B)$ a minimal projection. Let also $G_{1}=\left\{h \in G_{0} \mid \sigma_{0}(h)(b)=b, \forall b \in B\right\}$. It is immediate to check that all conditions $(i),(i i)$ are satisfied.

Q.E.D.

\section{Conjugacy CRiteria FOR ACTIONS}

We now prove a very general conjugacy (or isomorphism) criterion for actions of groups. Thus, we show that under very general conditions if a group measure space factor $M$ has two crossed product decompositions $M=A \rtimes_{\sigma} G=A_{0} \rtimes_{\sigma_{0}} G_{0}$ in a way that the group algebras $L(G), L\left(G_{0}\right)$ coincide, then modulo conjugacy by an inner automorphism of $M$ the two decompositions are identical ! The result is in fact surprisingly more general, requiring only the inclusion of one group algebra into the other and allowing amplifications.

We recall that if $K \triangleleft G$ is a normal subgroup and $\kappa \in \operatorname{Char}_{G}(K)$ is an $\operatorname{Ad}(G)$ invariant character of $K$ then one associates to it the scalar 2-cocycle $\mu_{\kappa} \in \mathrm{H}^{2}(G / K, \mathbb{T})$ as follows: Denote by $g \mapsto g^{\prime}$ a lifting of $G / K$ into $G$ then for $g_{1}, g_{2} \in G / K$ put $\mu_{\kappa}\left(g_{1}, g_{2}\right) \stackrel{\text { def }}{=} \kappa\left(g_{1}^{\prime} g_{2}^{\prime}\left(\left(g_{1} g_{2}\right)^{\prime}\right)^{-1}\right)$. It is easy to verify that $\mu_{\kappa}$ doesn't depend on the lifting and that it defines indeed a 2-cocycle on $G / K$.

5.1. Theorem. With the general notations 4.1, assume $\sigma$ is clustering and there exists a projection $p \in L(G)$ such that $p M p=M_{0}, p L(G) p \supset L\left(G_{0}\right)$. Then $p$ is central in $L(G), \tau(p)^{-1}$ is an integer, $p L(G) p=L\left(G_{0}\right)$ and there exist a normal subgroup $K \subset G$ with $|K|=\tau(p)^{-1}$, an $\operatorname{Ad}(G)$-invariant character $\kappa$ of $K$, with trivial $\mu_{\kappa} \in H^{2}(G / K)$, and a unitary element $u \in p L(G) p$ such that:

(i). $p=|K|^{-1} \Sigma_{k \in K} \kappa(k) u_{k}$ and $u_{k} p=\kappa(k) p, \forall k \in K$.

(ii). $u A_{0} u^{*}=A^{K} p$, where $A^{K}=\left\{a \in A \mid \sigma_{k}(a)=a, \forall k \in K\right\}$.

(iii). $\left\{u_{g} p \mid g \in G\right\}=\left\{u u_{h}^{0} u^{*} \mid h \in G_{0}\right\}$ modulo multiplication by scalars. More precisely, there exist an isomorphism $\delta: G_{0} \simeq G / K$, a lifting $G / K \ni g \mapsto g^{\prime} \in G$ 
and a map $\alpha: G_{0} \simeq G / K \rightarrow \mathbb{T}$ such that $\operatorname{Ad}(u)\left(u_{h}^{0}\right)=\overline{\alpha(h)} u_{\delta(h)^{\prime}} p, \forall h \in G_{0}$, and $\partial \alpha=\mu_{\kappa}$.

If in addition $G$ has no non-trivial finite normal subgroups (e.g. G ICC or torsion free) then $K=\{e\}, p=1$ and $\delta$ is an isomorphism $\delta: G_{0} \simeq G$.

Proof. Since ICC groups have no normal subgroups other than $K=\{e\}$, the last part follows trivially from $(i)$ and $(i i i)$.

By Theorem 4.2, the conditions in the hypothesis imply the existence of a partial isometry $v \in M$ with $v^{*} v=p$ and $v A_{0} v^{*}=A v v^{*}$. We are thus reduced to proving 5.1. $(i)-($ iii $)$ when this last condition is added to the list of assumptions. Notice also that by 3.7 the clustering condition on $\sigma$ implies $\sigma_{0}$ mixing. We in fact prove the following more general result:

5.2. Theorem. With the general notations 4.1, assume there exists a projection $p \in L(G)$ such that:

(a). $p=1_{M_{0}}, p M p \supset M_{0}, p L(G) p \supset L\left(G_{0}\right)$.

(b). One of the following holds true: $p=1, \sigma_{0}$ weakly mixing; or $p$ arbitrary, $\sigma_{0}$ weakly mixing, $\sigma$ mixing.

(c). There exists $v \in M$ such that $v^{*} v=p$ and $v A_{0} v^{*}=A v v^{*}$.

Then $\tau(p)^{-1}$ is an integer and there exist a subgroup $G^{\prime} \subset G$, a normal subgroup $K \subset G^{\prime}$ with $|K|=\tau(p)^{-1}$, a character $\gamma$ of $G_{0}$, an $\operatorname{Ad}\left(G^{\prime}\right)$-invariant character $\kappa$ of $K$, with trivial $\mu_{\kappa} \in H^{2}\left(G^{\prime} / K\right)$, and a unitary element $u \in p L(G) p$ such that $p^{\prime}=$ upu $^{*}$ is central in $L\left(G^{\prime}\right)=\left\{u_{g} \mid g \in G^{\prime}\right\}^{\prime \prime}$ and we have:

(i). $p^{\prime}=|K|^{-1} \Sigma_{k \in K} \kappa(k) u_{k} \in \mathcal{Z}\left(L\left(G^{\prime}\right)\right), u_{k} p^{\prime}=\kappa(k) p^{\prime}, \forall k \in K$.

(ii). $u A_{0} u^{*}=A^{K} p^{\prime}=p^{\prime} A p^{\prime}$, where $A^{K}=\left\{a \in A \mid \sigma_{k}(a)=a, \forall k \in K\right\}$.

(iii). $\left\{u_{g} p^{\prime} \mid g \in G^{\prime}\right\}=\left\{u u_{h}^{0} u^{*} \mid h \in G_{0}\right\}$ modulo multiplication by scalars. More precisely, there exist an isomorphism $\delta: G_{0} \simeq G^{\prime} / K$, a lifting $G^{\prime} / K \ni g \mapsto$ $g^{\prime} \in G^{\prime}$ and $\alpha: G_{0} \simeq G^{\prime} / K \rightarrow \mathbb{T}$ such that $\operatorname{Ad}(u)\left(u_{h}^{0}\right)=\overline{\alpha(h)} u_{\delta(h)^{\prime}} p^{\prime}, \forall h \in G_{0}$, and $\partial \alpha=\mu_{\kappa}$.

If in addition $p M p=M_{0}$ and $G$ has no non-trivial finite normal subgroups (e.g. $G$ is $I C C$ or torsion free), then condition (b) is redundant, $K=\{e\}, p=1, G^{\prime}=G$ and $\delta$ is an isomorphism $\delta: G_{0} \simeq G$.

Proof. Let us first prove the last part of the statement, assuming we have proved $(i)-($ iii $)$ under conditions $(a)-(c)$. Thus, we are under the additional assumptions $p M p=M_{0}$ and $G$ is ICC. By Lemma 4.5, there exists a subgroup $G_{1} \subset G_{0}$ of index $n<\infty$ and a projection $p_{1} \in \mathcal{P}\left(A_{0}\right)$ of trace $\tau\left(p_{1}\right)=\tau(p) / n$ that commutes 
with $\left\{u_{h}^{0}\right\}_{h \in G_{1}}$ such that if we denote $A_{1}=A_{0} p_{1}, \sigma_{1}(h)=\sigma_{0}(h)_{\mid A_{1}}$ then $\sigma_{1}$ is mixing. But then conditions $(a)-(c)$ are satisfied for $\sigma_{1}, G_{1}, p_{1}, v_{1}=v p_{1}$ instead of $\sigma_{0}, G_{0}, p, v$. Thus, by $(i)-(i i i)$ there exist $G_{1}^{\prime} \subset G$, with a finite normal subgroup $K_{1} \subset G_{1}^{\prime}$ satisfying $\left|K_{1}\right|=\tau\left(p_{1}\right)^{-1}$, and a unitary element $u_{1} \in p_{1} L(G) p_{1}$ such that $p_{1}^{\prime}=u_{1} p_{1} u_{1}^{*}$ is central in $L\left(G_{1}^{\prime}\right)$ and $u_{1} A_{1} u_{1}^{*}=A^{K_{1}} p_{1}^{\prime}=p_{1}^{\prime} A p_{1}^{\prime}$. Thus, after conjugation by $u_{1}$ we may assume $p_{1}$ satisfies $A_{0}=A^{K_{1}} p_{1}=p_{1} A p_{1}$ and $L\left(G_{1}\right)=p_{1} L\left(G_{1}^{\prime}\right) p_{1}=L\left(G_{1}^{\prime}\right) p_{1}$. But $\operatorname{sp} L\left(G_{1}\right) A_{1}$ is dense in $M_{1}$ and $p_{1}\left(A \rtimes G_{1}^{\prime}\right) p_{1}=$ $\overline{\mathrm{sp}} A^{K_{1}} L\left(G_{1}^{\prime}\right) p_{1}$. Since $p_{1} M p_{1}=M_{1}$, by expecting these equalities on $L(G)$ we get $L\left(G_{1}^{\prime}\right) p_{1}=p_{1} L(G) p_{1}$, which in particular implies $\left[G: G^{\prime}\right]<\infty$. But then $G$ ICC implies $G_{1}^{\prime}$ ICC (indeed, for if $G_{1}^{\prime}$ would have a non-trivial element $h$ with finite conjugacy class in $G_{1}^{\prime}$ then $h$ would also have finite conjugacy class in $G$, due to $\left.\left[G: G_{1}^{\prime}\right]<\infty\right)$. Thus $K_{1}=\{e\}$ forcing $p_{1}=1$. From $L\left(G_{1}^{\prime}\right) p_{1}=p_{1} L(G) p_{1}$ we also get $G_{1}^{\prime}=G$ and $\delta$ follows an isomorphism because $K_{1}=\{e\}$.

The proof of $(i)-($ iii $)$ will take the rest of this Section, as well as Section 6 . The following observation will be used several times:

(5.2.0). If $u^{\prime}$ is a partial isometry in $L(G)$ with $u^{\prime *} u^{\prime}=p$ and we substitute $p$ by $u^{\prime} u^{\prime *}, L\left(G_{0}\right)$ by $u^{\prime} L\left(G_{0}\right) u^{\prime *}, M_{0}$ by $u^{\prime} M_{0} u^{\prime *}, v$ by $v u^{\prime *}$ and $A_{0}$ by $u^{\prime} A_{0} u^{\prime *}$ then conditions $(a)-(c)$ are still satisfied.

We first prove the result assuming the Fourier expansion $v=\Sigma_{g} a_{g} u_{g}$ is finitely supported, with all $a_{g} \in A$ having finite spectrum. Under this assumption the proof simplifies, allowing the ideas to become more transparent. The proof of the general case is postponed to the next section.

Since all $a_{g}$ have finite spectrum and there are only finitely many of them, there exists $q \neq 0$ in $\mathcal{P}(A), q \leq v v^{*}$, such that $q a_{g}=c_{g} q, \forall g$, for some scalars $c_{g} \in \mathbb{C}$. Thus, if we denote $w=\Sigma_{g} c_{g} u_{g}$, then $w \in L(G)$ and $q w=q v$. Since $v^{*}(A q) v \subset A_{0}$ it follows that $w^{*} q A q w \subset A_{0}$. But $A$ is perpendicular to $L(G)$ and by Lemma 4.4 $A_{0}$ is also perpendicular to $p L(G) p$. Consequently,

$$
\tau(q) / \tau(p) p=E_{L(G)}\left(v^{*} q v\right)=E_{L(G)}\left(w^{*} q w\right)=w^{*} E_{L(G)}(q) w=\tau(q) w^{*} w .
$$

Hence, $u^{\prime}=\tau(p)^{-1 / 2} w$ is a partial isometry in $L(G)$ with $u^{\prime *} u^{\prime}=p$. Moreover, it satisfies $q v u^{\prime *}=q w u^{\prime *}=\tau(p)^{-1 / 2} u^{\prime} u^{\prime *}$. Thus, by the remark at the beginning of the proof it follows that in addition to conditions 5.2. $(a)-(c)$ we may also assume:

$$
q v=\tau(p)^{-1 / 2} q p
$$

We will prove that in fact conditions 5.2.(a) - (c) together with (5.2.1) imply 5.2. $(i)-($ iii $)$ for $u=p$. 
Since $u_{h}^{0}$ normalizes $A_{0}$ and $v A_{0} v^{*}=A v v^{*}, v u_{h}^{0} v^{*}$ is in the normalizing groupoid of $A$. Thus $q v u_{h}^{0} v^{*} q$ is also in the normalizing groupoid of $A$, implying that $q v u_{h}^{0} v^{*} q=\Sigma_{g} a_{g}^{h} u_{g}$ for some partial isometries $a_{g}^{h} \in A$. On the other hand $u_{h}^{0} \in L\left(G_{0}\right) \subset p L(G) p$, so $u_{h}^{0}=\Sigma_{g} c_{g}^{h} u_{g}$ for some scalars $c_{g}^{h}$. Together with $q v=\tau(p)^{-1 / 2} q p$ this implies:

$$
\Sigma_{g} a_{g}^{h} u_{g}=q v u_{h}^{0} v^{*} q=\tau(p)^{-1} q u_{h}^{0} q=\tau(p)^{-1} \Sigma_{g} c_{g}^{h} q \sigma_{g}(q) u_{g} .
$$

Identifying the coefficients of the most left and right Fourier expansions in (5.2.2), it follows that $a_{g}^{h}=\tau(p)^{-1} c_{g}^{h} q \sigma_{g}(q), \forall g$. In particular, if we denote $F^{h}=\{g \in G \mid$ $\left.a_{g}^{h} \neq 0\right\}=\left\{g \in G \mid c_{g}^{h} \neq 0, \sigma_{g}(q) q \neq 0\right\}$, then $g \in F^{h}$ implies $1=\left\|a_{g}^{h}\right\|=$ $\tau(p)^{-1}\left|c_{g}^{h}\right|$, and thus $\left|c_{g}^{h}\right|=\tau(p)$. Since $\tau(p)=\left\|u_{h}^{0}\right\|_{2}^{2}=\Sigma_{g}\left|c_{g}^{h}\right|^{2}$, this also shows that $\left|F^{h}\right| \leq \tau(p)^{-1}$. Summarizing:

$$
\left|c_{g}^{h}\right|=\tau(p), \forall g \in F^{h} ; \Sigma_{g}\left|c_{g}^{h}\right|^{2}=\tau(p) ;\left|F^{h}\right| \leq \tau(p)^{-1} .
$$

When $p=1$ then $q v=q, A q=A_{0} q$, and (5.2.3) shows that if $F^{h} \neq \emptyset$ then $F^{h}$ is a single point set $\{g(h)\}$, with $c^{h}=c_{g(h)}^{h}$ satisfying $\left|c^{h}\right|=1$ and $u_{h}^{0}=c^{h} u_{g(h)}$. Denote by $G_{1} \subset G_{0}$ the subgroup of elements $h \in G_{0}$ with $u_{h}^{0}$ a scalar multiple of some $u_{g(h)}$. If $h_{0} \in G_{0}$ is given, then by the weak mixing property of $\sigma_{0}$ there exists $h_{n} \in G_{0}$ such that $\lim _{n \rightarrow \infty} \tau\left(\sigma_{0}\left(h_{n}\right)(q) q\right)=\tau(q)^{2}$ and $\lim _{n \rightarrow \infty} \tau\left(\sigma_{0}\left(h_{n} h_{0}\right)(q) q\right)=\tau(q)^{2}$. Noticing that for $h^{\prime} \in G_{0}$ we have $F^{h^{\prime}} \neq \emptyset \Leftrightarrow q u_{h^{\prime}}^{0} q \neq 0 \Leftrightarrow \sigma_{0}\left(h^{\prime}\right)(q) q \neq 0$, it follows that $h_{n}, h_{n} h_{0} \in G_{1}$, which in turn implies $h \in G_{1}$. This shows that $G_{0}=G_{1}$. Finally notice that $A q=A_{0} q$ implies

$$
A_{0} \sigma_{0}(h)(q)=u_{h}^{0}\left(A_{0} q\right) u_{h}^{0 *}=u_{g(h)}(A q) u_{g(h)}^{*}=A \sigma_{g(h)}(q)
$$

and that by the ergodicity of $\sigma_{0}$ we have $1=\vee_{h} \sigma_{0}(h)(q)=\vee_{h} \sigma_{g(h)}(q)$. This shows that $A=A_{0}$ and letting $G^{\prime}=\left\{g(h) \mid h \in G_{0}\right\}$, 5.2.(i) - (iii) follow trivially.

Assume now $\sigma$ is mixing but $p$ is arbitrary. Notice that if for some $h$ we have $\left|F^{h}\right|=\tau(p)^{-1}$ then (5.2.3) forces $F^{h}$ to be equal to the support set $S^{h}=\{g \in G \mid$ $\left.c_{g}^{h} \neq 0\right\}$ of the Fourier expansion $u_{h}^{0}=\Sigma_{g} c_{g}^{h} u_{g}$, and $\left|c_{g}^{h}\right|=\tau(p), \forall g \in S^{h}$. We will prove that this is in fact the case for $\forall h \in G_{0}$, but first show it is true for all $h$ outside a finite subset of $G_{0}$.

By the mixing property, there exists $E \subset G$ finite such that $\tau\left(\sigma_{g}(q) q\right) \geq \tau(q)^{2} / 2$, $\forall g \in G \backslash E$. Define $\varepsilon_{0}>0$ to be the distance from $\tau(p)^{-1}$ to the closest integer $\neq \tau(p)^{-1}$. Since $u_{h}^{0}$ tends to 0 in the weak operator topology, as $h \rightarrow \infty$, there exists $E_{0} \subset G_{0}$ finite such that $\left|c_{g}^{h}\right|^{2}=\left|\tau\left(u_{h}^{0} u_{g}^{*}\right)\right|^{2}<\varepsilon_{0} \tau(p)^{2} /(2|E|+2), \forall g \in E$, 
$\forall h \in G_{0} \backslash E_{0}$. Thus, if $h \in G_{0} \backslash E_{0}$ and $g \in G \backslash E$ then $g \in S^{h} \Leftrightarrow g \in F^{h} \Leftrightarrow$ $\left|c_{g}^{h}\right|=\tau(p)$ (the last equivalence by $(5.2 .3)$ ).

If for some $h \in G_{0} \backslash E_{0}$ we have $\left|F^{h}\right|<\tau(p)^{-1}$, then from the above we get

$$
\begin{gathered}
\tau(p)=\Sigma_{g}\left|c_{g}^{h}\right|^{2}=\Sigma_{g \in E}\left|c_{g}^{h}\right|^{2}+\Sigma_{g \in F^{h}}\left|c_{g}^{h}\right|^{2} \\
=\Sigma_{g \in E}\left|c_{g}^{h}\right|^{2}+\left|F^{h}\right| \tau(p)^{2}<\varepsilon_{0} \tau(p)^{2} / 2+\left|F^{h}\right| \tau(p)^{2} \\
=\left(\varepsilon_{0} / 2+\left|F^{h}\right|\right) \tau(p)^{2} \leq\left(\tau(p)^{-1}-\varepsilon_{0} / 2\right) \tau(p)^{2}=\tau(p)-\varepsilon_{0} \tau(p)^{2} / 2,
\end{gathered}
$$

a contradiction. We have thus shown that $\tau(p)^{-1}$ is an integer and that for $h \in$ $G_{0} \backslash E_{0}$ we have: $S^{h}$ has $\tau(p)^{-1}$ elements; $\left|c_{h}^{g}\right|=\tau(p), \forall g \in S^{h}$. Conclusions $(i)$ and $($ iii) in 5.2 follow then from the following general fact:

5.3. Lemma. Let $G$ be a discrete group, $p \in L(G)=\left\{u_{g}\right\}_{g}{ }^{\prime \prime}$ a projection with $\tau(p)=1 / n$ for some integer $n \geq 1$. Let $\mathcal{W} \subset p L(G) p$ denote the set of unitary elements $w \in p L(G) p$ such that $S^{w}=F^{w}$, where $S^{w}=\left\{g \in G \mid c_{g}^{w} \neq 0\right\}$, $F^{w}=\left\{g \in G|| c_{g}^{w} \mid=\tau(p)\right\}, c_{g}^{w}$ being the coefficients of the Fourier expansion $w=$ $\Sigma_{g} c_{g}^{w} u_{g}$. Assume $\mathcal{W} \neq \emptyset$. Then $\mathcal{W}$ is a subgroup of $\mathcal{U}(p L(G) p)$ with neutral element equal to $p$ and there exist a subgroup $G^{\prime} \subset G$, a normal subgroup $K \subset G^{\prime}$ with $|K|=n$ and an $\operatorname{Ad}\left(G^{\prime}\right)$-invariant character $\kappa$ of $K$ such that $p=n^{-1} \Sigma_{k \in K} \kappa(k) u_{k}$, $u_{k} p=\kappa(k) u_{k}, \forall k \in K$, and $\mathcal{W}=\left\{\alpha u_{t} p \mid t \in G^{\prime}, \alpha \in \mathbb{T}\right\}$. Moreover, if $G^{\prime} / K \ni$ $h \mapsto h^{\prime} \in G^{\prime}$ is a lifting, then $\left\{u_{h^{\prime}} p \mid h \in G^{\prime} / K\right\}$ is a copy of the projective left regular representation of $G^{\prime} / K$ with scalar 2 -cocycle $\mu_{\kappa} \in \mathrm{H}^{2}\left(G^{\prime} / K\right)$.

Proof. Since $\mathcal{W} \cdot \mathcal{W} \subset \mathcal{U}(p L(G) p)$ and any $u \in \mathcal{U}(p L(G) p)$ satisfies $\tau\left(u^{*} u\right)=\tau(p)$, it follows that for all $w, w^{\prime} \in \mathcal{W}$ we have:

$$
\left|F^{w}\right|=n ; \Sigma_{g}\left|c_{g}^{w}\right|^{2}=\tau(p)=\Sigma_{t}\left|c_{t}^{w w^{\prime}}\right|^{2} ; \Sigma_{g}\left|c_{g}^{w}\right|=1
$$

We also have

$$
w w^{\prime}=\Sigma_{t \in S^{w} w^{\prime}}\left(\Sigma_{g \in S^{w}} c_{g}^{w} c_{g^{-1} t}^{w^{\prime}}\right) u_{t}=\Sigma_{t} c_{t}^{w w^{\prime}} u_{t}
$$

and there are at most $n^{2}$ non-zero elements $c_{t}^{w w^{\prime}}, t \in G$, and they satisfy the trivial inequalities

$$
\begin{aligned}
& \left|c_{t}^{w w^{\prime}}\right| \leq \Sigma_{g}\left|c_{g}^{w}\right|\left|c_{g^{-1} t}^{w^{\prime}}\right| \leq \tau(p) \\
& \Sigma_{t}\left|c_{t}^{w w^{\prime}}\right| \leq \Sigma_{g, g^{\prime}}\left|c_{g}^{w}\right|\left|c_{g^{\prime}}^{w^{\prime}}\right|=1
\end{aligned}
$$


Moreover, the first inequality in (5.3.2) becomes equality iff $c_{g}^{w} c_{g^{-1} t}^{w^{\prime}}, g \in G$, are all equal, when non-zero, while the second inequality in (5.3.2) becomes equality iff $g \mapsto g^{-1} t$ is a bijection from $S^{w}$ to $S^{w^{\prime}}$.

Notice that the maximum of the expression $\Sigma_{i=1}^{n^{2}} x_{i}^{2}$ over the set of $n^{2}$-tuples $\left\{\left(x_{i}\right)_{i} \mid 0 \leq x_{i} \leq 1 / n, \forall i, \Sigma_{j} x_{j} \leq 1\right\}$ is equal to $1 / n$ and it is attained when $n$ of the $x_{i}$ 's are equal to $1 / n$ and all others are equal to 0 . Indeed, this is because

$$
x_{i}^{2}+x_{j}^{2} \leq\left(\min \left\{x_{i}+x_{j}, 1 / n\right\}\right)^{2}+\left(\max \left\{0, x_{i}+x_{j}-1 / n\right\}\right)^{2},
$$

with equality if and only if $\left\{x_{i}, x_{j}\right\}=\left\{\min \left\{x_{i}+x_{j}, 1 / n\right\}, \max \left\{0, x_{i}+x_{j}-1 / n\right\}\right\}$.

Applying this to $\left(x_{i}\right)_{i}=\left(\left|c_{g}^{w w^{\prime}}\right|\right)_{g}$, it follows that $\Sigma_{t}\left|c_{t}^{w w^{\prime}}\right|^{2} \leq 1 / n=\tau(p)$ with equality iff there are exactly $n$ non-zero elements $c_{t}^{w w^{\prime}}$ and they all have absolute value $\tau(p)$, i.e. iff $S^{w w^{\prime}}=F^{w w^{\prime}}$. Since (5.3.1) shows that the equality does hold true, it follows that $w w^{\prime} \in \mathcal{W}$. In addition, the conditions for equality in (5.3.2) are verified, i.e.

$$
S^{w^{\prime}}=\left(S^{w}\right)^{*} t, c_{g}^{w} c_{g^{-1} t}^{w^{\prime}}=c_{t}^{w w^{\prime}} \tau(p), \forall w, w^{\prime} \in \mathcal{W}, t \in S^{w w^{\prime}}, g \in S^{w}
$$

Since we clearly have $\mathcal{W}^{*}=\mathcal{W}$, this shows that $p=w w^{*}$ belongs to $\mathcal{W}$ and that $\mathcal{W}$ is a group with $p$ as neutral element. Denote $K=S^{p}$ and note that $K=K^{*}$. By (5.3.4) applied to $w=p=w^{\prime}$, it follows that $K$ is a group with $n$ elements. Since $p$ is a projection of the form $p=|K|^{-1} \Sigma_{k \in K} \alpha_{k} u_{k}$, with $\alpha_{k}=c_{k}^{p} / \tau(p)$ having absolute value $1, \forall k \in K, \kappa(k) \stackrel{\text { def }}{=} \alpha_{k}, k \in K$, gives a character of $K$.

Also, by (5.3.4) applied first to $w=p$, then to $w^{\prime}=p$, it follows that for all $w \in \mathcal{W}$ there exist $t, t^{\prime} \in G$ with $S^{w}=K t=t^{\prime} K$. This implies $K t K=t K$, so $t$ normalizes $K$. Thus, if we denote $G^{\prime}=\left\{t \in G \mid \exists w \in \mathcal{W}, S^{w}=K t\right\}$ then $G^{\prime}$ follows a group with $K \subset G^{\prime}$ a normal subgroup. Moreover, $w$ is a scalar multiple of $p u_{t}$ and $\left[u_{t}, p\right]=0, \forall t \in G^{\prime}$. In particular, the character $\kappa$ of $K$ must be $\operatorname{Ad}\left(G^{\prime}\right)$ invariant and it satisfies $u_{k} p=\kappa(k) p, \forall k \in K$. The last part of the statement is now trivial, since $\tau\left(u_{g} p\right)=0, \forall g \in G^{\prime} \backslash K$ and the 2-cocycle $\mu_{\kappa} \in \mathrm{H}^{2}\left(G^{\prime} / K\right)$ satisfies $u_{\left(h_{1}\right)^{\prime}} p^{\prime} u_{\left(h_{2}\right)^{\prime}} p^{\prime}=\mu_{\kappa}\left(h_{1}, h_{2}\right) u_{\left(h_{1} h_{2}\right)^{\prime}} p^{\prime}$ by the way it is defined. Q.E.D.

We can now end the proof of 5.2 under the extra assumption that the Fourier expansion $v=\Sigma_{g} a_{g} u_{g}$ is finitely supported, with all $a_{g}$ having finite spectrum. Thus, by the formula $p=\Sigma_{k \in K} \kappa(k) u_{k}$ it follows that $A^{K}=A \cap\{p\}^{\prime}$ with $p$ a Jones projection implementing the $\tau$-preserving conditional expectation $E_{A^{K}}$ of $A$ onto $A^{K}$. Since $q v=\tau(p)^{-1 / 2} q p$, the projection $q \in A$ satisfies $p q p=E_{A^{K}}(q) p=\tau(p) q^{\prime} p$ for some unique projection $q^{\prime} \in A^{K}$. By (5.2.1) we thus get

$$
A_{0} q^{\prime} p=A_{0}(p q p)=A_{0}\left(v^{*} q v\right)=v^{*}(A q) v=p(A q) p=A^{K} q^{\prime} p
$$


The equality $A_{0} p=A^{K} p$ in 5.2.(ii) follows now from this identity, the ergodicity of $\sigma_{0}$ on $A_{0}$ and the inclusion $\left\{u_{h}^{0}\right\}_{h \in G_{0}} \subset\left\{\alpha u_{g} p \mid g \in G^{\prime}, \alpha \in \mathbb{T}\right\}$, the same way we deduced the similar equality in the case $p=1$ in (5.2.4). Part 5.2.(iii) is an immediate consequence of the last part of Lemma 5.3.

\section{END OF PROOF OF 5.2}

We now prove 5.2 in general, without any extra-assumption on the intertwiner $v=\Sigma_{g} a_{g} u_{g}$. The idea is to prove first an approximate version of condition (5.2.1), and then re-do steps (5.2.2) - (5.2.5) using it in-lieu of (5.2.1).

6.1. Lemma. Let $(A, \tau), G, \sigma, M=A \rtimes_{\sigma} G,\left\{u_{g}\right\}_{g}$ be as in 4.1. Let $p \in \mathcal{P}(L(G))$ and assume $v \in M$ is a partial isometry with $v^{*} v=p, v v^{*} \in A$ and $v^{*} A v$ perpendicular to $L(G)$. Then there exists a partial isometry $u^{\prime} \in L(G)$ and a decreasing sequence of non-zero projections $q_{n} \in A v v^{*}$ such that $u^{\prime *} u^{\prime}=p$ and

$$
\left\|q v-\tau(p)^{-1 / 2} q u^{\prime}\right\|_{2}^{2}<2^{-n} \tau(q), \forall q \in \mathcal{P}\left(A q_{n}\right)
$$

Proof. Let $v=\Sigma_{g} a_{g} u_{g}$ be the Fourier expansion of $v$ in $M=A \rtimes_{\sigma} G$ and denote $f_{0}=v v^{*} \in \mathcal{P}(A)$. Since the infinite sum $\Sigma_{g} a_{g} a_{g}^{*}$ is increasing and tends to $f_{0}$ in the strong operator topology, or equivalently in $\|\cdot\|_{1}$, there exists $f \neq 0$ in $\mathcal{P}\left(A f_{0}\right)$ such that $\Sigma_{g} a_{g} a_{g}^{*} f$ is convergent to $f$ in the uniform norm. Thus, there exists an increasing sequence of finite sets $F_{n} \subset G$ such that $\cup_{n} F_{n}=G$ and

$$
\left\|\Sigma_{g \notin F_{n}} a_{g} a_{g}^{*} f\right\| \leq 2^{-n-3}
$$

By the Gelfand-Neimark theorem $A f=C(\Omega)$ for some compact set $\Omega$. Choose $\omega_{0} \in \Omega$ and denote $c_{g}=a_{g}\left(\omega_{0}\right), g \in G$. Thus $\Sigma_{g} c_{g} \overline{c_{g}}=1$, implying that $w=\Sigma_{g} c_{g} u_{g}$ belongs to $L^{2}(L(G))=\ell^{2}(G)$. By the continuity of the functions $a_{g} \in C(\Omega)$ at $\omega_{0}$, we can choose recursively $q_{n} \in \mathcal{P}(A f)$ (corresponding to open-closed neighborhoods of $\omega_{0}$ in $\left.\Omega\right)$ such that $q_{n} \leq q_{n-1}$ and

$$
\left\|a_{g} q_{n}-c_{g} q_{n}\right\| \leq 2^{-n-2}\left|F_{n}\right|^{-1 / 2}, \forall g \in F_{n}
$$

Using first the Cauchy-Schwartz inequality and then (6.1.2) we get for any $q \in$ $\mathcal{P}\left(A q_{n}\right)$ :

$$
\begin{gathered}
\left|\Sigma_{g \in F_{n}} \tau\left(\overline{c_{g}}\left(a_{g}-c_{g}\right) q\right)\right|=\left|\Sigma_{g \in F_{n}} \tau\left(\overline{c_{g}} q\left(a_{g}-c_{g}\right) q\right)\right| \\
\leq\left(\Sigma_{g \in F_{n}}\left\|c_{g} q\right\|_{2}^{2}\right)^{1 / 2}\left(\Sigma_{g \in F_{n}}\left\|\left(a_{g}-c_{g}\right) q\right\|_{2}^{2}\right)^{1 / 2}
\end{gathered}
$$




$$
\begin{aligned}
& \leq\left(\Sigma_{g \in F_{n}}\left|c_{g}\right|^{2} \tau(q)\right)^{1 / 2}\left(\Sigma_{g \in F_{n}} 2^{-2 n-4}\left|F_{n}\right|^{-1} \tau(q)\right)^{1 / 2} \\
& \leq\left(\Sigma_{g \in G}\left|c_{g}\right|^{2} \tau(q)\right)^{1 / 2} 2^{-n-2} \tau(q)^{1 / 2}=2^{-n-2} \tau(q) .
\end{aligned}
$$

Similarly, by first using the Cauchy-Schwartz inequality and then (6.1.1) we have:

$$
\begin{gathered}
\left|\tau\left(\Sigma_{g \notin F_{n}} \overline{c_{g}} a_{g} q\right)\right| \\
\leq\left(\Sigma_{g \notin F_{n}}\left|c_{g}\right|^{2} \tau(q)\right)^{1 / 2}\left(\tau\left(\Sigma_{g \notin F_{n}} a_{g} a_{g}^{*} q\right)\right)^{1 / 2} \leq 2^{-n-3} \tau(q) .
\end{gathered}
$$

Using (6.1.3) and (6.1.4) we finally obtain the estimates:

$$
\|q w-q v\|_{2}^{2}=2 \tau(q)-2 \operatorname{Re} \tau\left(q v w^{*} q\right)
$$

$$
\begin{gathered}
=2 \tau(q)-2 \operatorname{Re} \tau\left(\Sigma_{g} \overline{c_{g}} a_{g} q\right)=2 \tau(q)-2 \operatorname{Re} \tau\left(\Sigma_{g \in F_{n}} \overline{c_{g}} a_{g} q\right)-2 \operatorname{Re} \tau\left(\Sigma_{g \notin F_{n}} \overline{c_{g}} a_{g} q\right) \\
=2 \tau(q)-2 \Sigma_{g \in F_{n}} c_{g} \overline{c_{g}} \tau(q)+2 \Sigma_{g \in F_{n}} \tau\left(\overline{c_{g}}\left(a_{g}-c_{g}\right) q\right)-2 \operatorname{Re} \tau\left(\Sigma_{g \notin F_{n}} \overline{c_{g}} a_{g} q\right) \\
\leq 2 \tau(q)-2 \tau(q)+2 \cdot 2^{-n-3} \tau(q)+2 \cdot 2^{-n-2} \tau(q)+2 \cdot 2^{-n-3} \tau(q)=2^{-n} \tau(q) .
\end{gathered}
$$

This shows that if we put $u^{\prime}=\tau(p)^{1 / 2} w$ and $q_{n}$ are chosen as above, then the inequality $(6.1)$ is satisfied $\forall q \in \mathcal{P}\left(A q_{n}\right)$.

We still have to prove that $u^{\prime *} u^{\prime}=p$. To this end, note that $w \in L^{2}(L(G))$ and $q_{n} \in A q_{n} \perp L(G)$ implies $E_{L(G)}\left(w^{*} q_{n} w\right)=w^{*} E_{L(G)}\left(q_{n}\right) w=\tau\left(q_{n}\right) w^{*} w$ (these calculations make sense in $\left.L^{1}(L(G))\right)$. On the other hand, $v^{*} q_{n} v \in v^{*}\left(A q_{n}\right) v \perp$ $L(G)$ by hypothesis, so that we also have $E_{L(G)}\left(v^{*} q_{n} v\right)=\left(\tau\left(q_{n}\right) / \tau(p)\right) p$. But by the Cauchy-Schwartz inequality and (6.1) we have

$$
\begin{gathered}
\left\|E_{L(G)}\left(w^{*} q_{n} w-v^{*} q_{n} v\right)\right\|_{1} \leq\left\|w^{*} q_{n} w-v^{*} q_{n} v\right\|_{1} \\
\leq\left\|w^{*} q_{n} w-w^{*} q_{n} v\right\|_{1}+\left\|w^{*} q_{n} v-v^{*} q_{n} v\right\|_{1} \\
\leq\left\|w^{*} q_{n}\right\|_{2}\left\|q_{n} w-q_{n} v\right\|_{2}+\left\|w^{*} q_{n}-v^{*} q_{n}\right\|_{2}\left\|q_{n} v\right\|_{2} \\
=2\left\|q_{n} w-q_{n} v\right\|_{2}\left\|q_{n}\right\|_{2} \leq 2^{-n+1} \tau\left(q_{n}\right) .
\end{gathered}
$$

Thus, for all $n \geq 1$ we have

$$
\left.\| \tau\left(q_{n}\right) / \tau(p)\right) p-\tau\left(q_{n}\right) w^{*} w\left\|_{1}=\right\| E_{L(G)}\left(w^{*} q_{n} w-v^{*} q_{n} v\right) \|_{1} \leq 2^{-n+1} \tau\left(q_{n}\right)
$$

implying that $u^{\prime}=\tau(p)^{1 / 2} w$ satisfies $\left\|u^{\prime *} u^{\prime}-p\right\|_{1} \leq \tau(p) 2^{-n+1}, \forall n$. Thus $u^{\prime *} u^{\prime}=p$. Q.E.D. 
Let now $u^{\prime} \in L(G)$ and $q_{n} \in \mathcal{P}\left(A v v^{*}\right)$ be as given by Lemma 6.1. Since for $q \in \mathcal{P}\left(A q_{n}\right)$ we have $\left\|q v u^{\prime *}-\tau(p)^{-1 / 2} q p\right\|_{2}=\left\|q v-\tau(p)^{-1 / 2} q u^{\prime}\right\|_{2} \leq 2^{-n}\|q\|_{2}$, by the remark at the beginning of the proof of 5.2 in Section 5 , we may assume there exists a decreasing sequence of non-zero projections $q_{n} \in A v v^{*}$ such that in addition to the assumptions 5.2.(a) - (c) we also have:

$$
\left\|q v-\tau(p)^{-1 / 2} q p\right\|_{2} \leq 2^{-n}\|q\|_{2}, \forall q \in \mathcal{P}\left(A q_{n}\right)
$$

We will prove that in fact conditions 5.2.(a) - (c) together with (6.2) imply 5.2. $(i)-($ iii $)$ for $u=p$. In particular, when $p=1$ this amounts to showing that $\left\{u_{h}^{0}\right\}_{h} \subset\left\{\alpha u_{g} \mid g \in G, \alpha \in \mathbb{T}\right\}$ and $A=A_{0}$.

As in Section 5, let $u_{h}^{0}=\Sigma_{g} c_{g}^{h} u_{g}$ be the Fourier expansion of $u_{h}^{0} \in L\left(G_{0}\right) \subset$ $p L(G) p$, with $c_{g}^{h} \in \mathbb{C}$. Since $u_{h}^{0}$ are unitaries in $p L(G) p$, we have $\tau(p)=\tau\left(u_{h}^{0} u_{h}^{0 *}\right)=$ $\Sigma_{g}\left|c_{g}^{h}\right|^{2}, \forall h \in G_{0}$. Note that $q_{n} u_{h}^{0} q_{n}=\Sigma_{g} c_{g}^{h} \sigma_{g}\left(q_{n}\right) q_{n} u_{g}$. We also consider the Fourier expansion $q_{n} v u_{h}^{0} v^{*} q_{n}=\Sigma_{g} a_{g}^{h, n} u_{g}$. Since the left term is in the normalizing groupoid of $A q_{n} \subset q_{n} M q_{n}$, it follows that $a_{g}^{h, n}, g \in G$, (resp. $\left.\sigma_{g^{-1}}\left(a_{g}^{h, n}\right), g \in G\right)$ are partial isometries in $A q_{n}$ with mutually orthogonal supports. But unfortunately we no longer have the equality $q_{n} v u_{h}^{0} v^{*} q_{n}=\tau(p)^{-1} q_{n} u_{h}^{0} q_{n}$ that would make possible the identification of the Fourier coefficients of the two sides, as in (5.2.2). However, an "approximation" of that equality does hold true due to (6.2), and this will be good enough for us to prove the following version of (5.2.3):

6.3. Lemma. Let $F_{n}^{h}=\left\{g \in G \mid a_{g}^{h, n} \neq 0\right\}$. For all $g \in F_{n}^{h}$ we have:

(i). $\left\|a_{g}^{h}-c_{g}^{h} \tau(p)^{-1} \sigma_{g}\left(q_{n}\right) q_{n}\right\| \leq 2^{-n+1}$ and if $n \geq 2$ then $\left|a_{g}^{h}\right|=\sigma_{g}\left(q_{n}\right) q_{n}$.

(ii). $\left|1-\tau(p)^{-1}\right| c_{g}^{h}|| \leq 2^{-n+1}$ and $\left|F_{n}^{h}\right| \leq \tau(p)^{-1}+2^{-n+2}$.

(iii). $\left|c_{g}^{h}\right| \leq \tau(p)$ and $F_{n}^{h} \supset F_{n+1}^{h}, \forall n$.

Proof. If $F_{n}^{h}=\emptyset$ then there is nothing to prove. If $F_{n}^{h}$ is non-empty then fix $g_{0} \in F_{n}^{h}$. Denote $\bar{q}=q_{n} \sigma_{g_{0}}\left(q_{n}\right)$ and note that $\left|a_{g_{0}}^{h, n}\right| \leq \bar{q}$. For each projection $q \in \mathcal{P}(A \bar{q})$ denote $q^{\prime}=\sigma_{g_{0}^{-1}}(q) \leq q_{n}$. Note that if $q^{\prime} \leq \bar{q}$ then $q u_{g_{0}} q^{\prime}=q u_{g_{0}}$ and if $q \leq\left|a_{g_{0}}^{h, n}\right|$ then $q v u_{h}^{0} v^{*} q^{\prime}=q a_{g_{0}}^{h, n} u_{g_{0}}$.

By (6.1) applied to $q, q^{\prime} \leq q_{n}$ we have $\left\|q v-\tau(p)^{-1 / 2} q p\right\|_{2} \leq 2^{-n}\|q\|_{2}$ and $\| v^{*} q^{\prime}-$ $\tau(p)^{-1 / 2} p q^{\prime}\left\|_{2} \leq 2^{-n}\right\| q^{\prime} \|_{2}$. Using this and the inequality $\|x y\|_{2} \leq\|x\|\|y\|_{2}$ we get:

$$
\begin{gathered}
\left\|q v u_{h}^{0} v^{*} q^{\prime}-\tau(p)^{-1} q u_{h}^{0} q^{\prime}\right\|_{2} \\
\leq\left\|q v u_{h}^{0} v^{*} q^{\prime}-\tau(p)^{-1 / 2} q u_{h}^{0} v^{*} q^{\prime}\right\|_{2}+\left\|\tau(p)^{-1 / 2} q u_{h}^{0} v^{*} q^{\prime}-\tau(p)^{-1} q u_{h}^{0} q^{\prime}\right\|_{2}
\end{gathered}
$$




$$
\begin{gathered}
\leq\left\|q v-\tau(p)^{-1 / 2} q p\right\|_{2}\left\|u_{h}^{0} v^{*} q^{\prime}\right\|+\tau(p)^{-1 / 2}\left\|q u_{h}^{0}\right\|\left\|v^{*} q^{\prime}-\tau(p)^{-1 / 2} p q^{\prime}\right\|_{2} \\
\leq 2^{-n}\|q\|_{2}+2^{-n}\left\|q^{\prime}\right\|_{2}=2^{-n+1}\|q\|_{2} .
\end{gathered}
$$

For any $d$ in the spectrum of $a_{g_{0}}^{h, n}$ in $A \bar{q}$ and any $\varepsilon>0$, take $0 \neq q \in \mathcal{P}(A \bar{q})$ to be a spectral projection of $a_{g_{0}}^{h, n}$ in $A \bar{q}$ such that $\left\|a_{g_{0}}^{h, n} q-d\right\| \leq \varepsilon$. Using (6.3.1) we then get:

$$
\begin{gathered}
2^{-n+1}\|q\|_{2} \geq\left\|q v u_{h}^{0} v^{*} q^{\prime}-\tau(p)^{-1} q u_{h}^{0} q^{\prime}\right\|_{2} \\
=\left\|\left(a_{g_{0}}^{h, n} q-\tau(p)^{-1} c_{g_{0}}^{h} q\right) u_{g_{0}}-\Sigma_{g \neq g_{0}} c_{g}^{h} q \sigma_{g}\left(q^{\prime}\right) u_{g}\right\|_{2} \\
\geq\left\|a_{g_{0}}^{h, n} q-\tau(p)^{-1} c_{g_{0}}^{h} q\right\|_{2} \geq\left\|d q-\tau(p)^{-1} c_{g_{0}}^{h} q\right\|_{2}-\varepsilon\|q\|_{2} \\
=\left|d-\tau(p)^{-1} c_{g_{0}}^{h}\right|\|q\|_{2}-\varepsilon\|q\|_{2} .
\end{gathered}
$$

Since $d \in \operatorname{spec}\left(a_{g_{0}}^{h, n}\right)$ and $\varepsilon>0$ were arbitrary, $\left\|a_{g_{0}}^{h, n}-\tau(p)^{-1} c_{g_{0}}^{h} \bar{q}\right\| \leq 2^{-n+1}$. This also shows that if $n \geq 2$ then $\left|a_{g_{0}}^{h, n}\right|=\bar{q}$. Moreover, $\left|1-\tau(p)^{-1}\right| c_{g_{0}}^{h}|| \leq$ $\left\|a_{g_{0}}^{h, n}-\tau(p)^{-1} c_{g_{0}}^{h} \bar{q}\right\| \leq 2^{-n+1}$.

From the equality $\Sigma_{g \in G}\left|c_{g}^{h}\right|^{2}=\tau(p)$ and the inequalities $\left|c_{g}^{h}\right| \geq \tau(p)\left(1-2^{-n+1}\right)$ we get

$$
\tau(p) \geq \Sigma_{g \in F_{n}^{h}}\left|c_{g}^{h}\right|^{2} \geq\left|F_{n}^{h}\right| \tau(p)^{2}\left(1-2^{-n+1}\right)^{2},
$$

showing that $\left|F_{n}^{h}\right| \leq \tau(p)^{-1}\left(1-2^{-n+1}\right)^{-2} \leq \tau(p)^{-1}\left(1+2^{-n+2}\right)$.

The sequence of sets $F_{n}^{h}$ follows decreasing in $n$ by the definition of $F_{n}^{h}$ and by the fact that the sequence $q_{n}$ is decreasing. Also, by the Cauchy-Schwartz inequality we have $\left|c_{g}^{h}\right|=\left|\tau\left(u_{g}^{0} u_{g}^{*}\right)\right| \leq\left\|u_{g}^{0}\right\|_{2}\left\|u_{g} p\right\|_{2}=\tau(p)$.

Q.E.D.

Proof of 5.2 in case $p=1, \sigma_{0}$ weakly mixing. Since $p=1$, condition (6.2) becomes:

$$
\|q v-q\|_{2} \leq 2^{-n}\|q\|_{2}, \forall q \in \mathcal{P}\left(A q_{n}\right)
$$

for some decreasing sequence of non-zero projections $q_{n}$ in $A v v^{*}$. By the weak mixing property of $\sigma_{0}$, there exists a sequence $\left\{h_{m}\right\}_{m} \in G_{0}$ such that

$$
\lim _{m \rightarrow \infty} \tau\left(\sigma_{0}\left(h_{m} h\right)\left(v^{*} q_{n} v\right) v^{*} q_{n} v\right)=\tau\left(q_{n}\right)^{2}, \forall n \geq 1, h \in G_{0} .
$$

Note that for $h^{\prime} \in G_{0}$ and $n \geq 1$ we have $F_{n}^{h^{\prime}} \neq \emptyset \Leftrightarrow q_{n} v u_{h^{\prime}}^{0} v^{*} q_{n} \neq 0 \Leftrightarrow$ $\sigma_{0}\left(h^{\prime}\right)\left(v^{*} q_{n} v\right) v^{*} q_{n} v \neq 0$. Let now $h \in G_{0}$ be an arbitrary element and $n \geq 3$. Taking $h^{\prime}=h_{m}$ and then $h^{\prime}=h_{m} h$ in these equivalences, with $m$ large enough, it follows by (6.4.2) that $F_{n}^{h_{m}}, F_{n}^{h_{m} h} \neq \emptyset$. By Lemma 6.3 we have $\left|F_{n}^{h_{m} h}\right|,\left|F_{n}^{h_{m}}\right| \leq$ $1+2^{-n+2} \leq 3 / 2$. Thus $F_{n}^{h_{m}}=\{g\}, F_{n}^{h_{m} h}=\left\{g^{\prime}\right\}$, for some $g=g\left(n, h_{m}\right), g^{\prime}=$ $g\left(n, h_{m} h\right)$. 
Moreover, by 6.3 again, there exist unit scalars $c, c^{\prime} \in \mathbb{T}$ such that $\left|c-c_{g}^{h_{n}}\right| \leq$ $2^{-n+2},\left|c^{\prime}-c_{g^{\prime}}^{h_{n} h}\right| \leq 2^{-n+2}$. Recalling that $u_{h_{n}}^{0}=\Sigma_{g} c_{g}^{h_{n}} u_{g}$, we get

$$
\begin{aligned}
& \left\|u_{h_{n}}^{0}-c u_{g}\right\|_{2}^{2}=\left|c_{g}^{h_{n}}-c\right|^{2}+\left(1-\left|c_{g}^{h_{n}}\right|^{2}\right) \\
& \leq 2^{-2 n+4}+2^{-n+2}\left(1+2^{-n+2}\right) \leq 2^{-n+3} .
\end{aligned}
$$

Similarly $\left\|u_{h_{n}}^{0}-c u_{g}\right\|_{2}^{2} \leq 2^{-n+3}$. Since $u_{h}^{0}=u_{h_{n}^{-1}}^{0} u_{h_{n} h}^{0}$, by the triangle inequality we deduce:

$$
\begin{gathered}
\left\|u_{h}^{0}-c^{-1} c^{\prime} u_{g^{-1} g^{\prime}}\right\|_{2} \\
\leq\left\|u_{h_{n}^{-1}}^{0} u_{h_{n} h}^{0}-u_{h_{n}^{-1}}^{0}\left(c^{\prime} u_{g^{\prime}}\right)\right\|_{2}+\| u_{h_{n}^{-1}}^{0}\left(c^{\prime} u_{g^{\prime}}\right)-\left(c^{-1} u_{g^{-1}}\right)\left(c^{\prime} u_{g^{\prime}} \|_{2}\right. \\
=\left\|u_{h_{n} h}^{0}-c^{\prime} u_{g^{\prime}}\right\|_{2}+\left\|u_{h_{n}^{-1}}^{0}-c^{-1} u_{g^{-1}}\right\|_{2} \leq 2^{(-n+5) / 2} .
\end{gathered}
$$

Since $n \geq 3$ can be taken arbitrarily large in these inequalities, independently of $h \in G_{0}$, this shows that $\left\{u_{h}^{0}\right\}_{h} \subset\left\{\alpha u_{g} \mid g \in G, \alpha \in \mathbb{T}\right\}$. Since $\left\{u_{h}^{0}\right\}_{h}$ is a representation of the group $G_{0}$, there exist a character $\gamma$ of $G_{0}$ and an isomorphism $\delta: G_{0} \simeq G^{\prime} \subset G$ such that $u_{h}^{0}=\gamma(h) u_{\delta(h)}, \forall h \in G_{0}$.

This proves all but $(i i)$ in 5.2. Proving $(i i)$ amounts to show that under the extraassumption (6.2), $A$ and $A_{0}$ follow equal. But by (6.2), under the "infinitesimal" projections $q_{n} \in A, v^{*} q_{n} v \in A_{0}$ the two algebras "almost" coincide. Since $\left\{u_{h}^{0}\right\}_{h}$ is contained in $\left\{u_{g}\right\}_{g}$ modulo the scalars and they act ergodically on $A_{0}$ resp. $A$, this local "almost coincidence" extends to a global "almost coincidence" of $A, A_{0}$, which due to the arbitrariness of the approximations shows that $A=A_{0}$. The full details of this argument are carried out in $(6.6 .1)-(6.6 .3)$, where the case " $p$ arbitrary" is covered.

Q.E.D.

Proof of 5.2 in the case $\sigma_{0}$ weakly mixing, $\sigma$ mixing. By 4.4. $(i)$, since $\sigma$ is mixing and $\sigma_{0}$ weakly mixing, it follows that $\sigma_{0}$ is mixing as well. Let $\varepsilon_{0}>0$ be the distance from $\tau(p)^{-1}$ to the closest integer $\neq \tau(p)^{-1}$ and take $0<\varepsilon \leq \varepsilon_{0}\left(1+\tau(p)^{-1}\right)^{-1} / 2$.

Since $\sigma$ is mixing there exists a finite subset $E \subset G$ such that

$$
\left|\tau\left(\sigma_{g}\left(q_{n}\right) q_{n}\right)-\tau\left(q_{n}\right)^{2}\right| \leq \varepsilon \tau\left(q_{n}\right)^{2}, \forall g \in G \backslash E .
$$

Similarly, since $\sigma_{0}$ is mixing and $\sigma_{0}(h)(y)=u_{h}^{0} y u_{h}^{0 *}, \forall y \in A_{0}=A p, h \in G_{0}$, by taking into account that $v^{*} q_{n} v \in A_{0}$ and $\tau_{M_{0}}(y)=\tau(p)^{-1} \tau(y), \forall y \in A_{0}=A p$, it follows that there exists $E_{0} \subset G_{0}$ finite such that

$$
\left|\tau\left(u_{h}^{0} v^{*} q_{n} v u_{h}^{0 *} v^{*} q_{n} v\right)-\tau(p)^{-1} \tau\left(q_{n}\right)^{2}\right| \leq \varepsilon \tau\left(q_{n}\right)^{2}, \forall h \in G_{0} \backslash E_{0} .
$$


Moreover, since $\left\{u_{h}^{0}\right\}_{h}$ tends weakly to zero as $h \rightarrow \infty$, it follows that we may also assume $E_{0}$ is large enough to ensure

$$
\left|c_{g}^{h}\right|=\left|\tau\left(u_{h}^{0} u_{g}^{*}\right)\right| \leq \varepsilon /|E|, \forall g \in E, \forall h \in G_{0} \backslash E_{0}
$$

But $q_{n} v u_{h}^{0} v^{*} q_{n}=\Sigma_{g} a_{g}^{h, n} u_{g}$, so using the fact that $\left\|\Sigma_{g} a_{g}^{h, n} u_{g}\right\|_{2}^{2}=\Sigma_{g} \tau\left(\left|a_{g}^{h, n}\right|\right)$ and $\left\|q_{n} v u_{h}^{0} v^{*} q_{n}\right\|_{2}^{2}=\tau\left(u_{h}^{0} v^{*} q_{n} v u_{h}^{0 *} v^{*} q_{n} v\right)$, by (6.5.2) it follows that

$$
\left|\Sigma_{g} \tau\left(\left|a_{g}^{h, n}\right|\right)-\tau(p)^{-1} \tau\left(q_{n}\right)^{2}\right| \leq \varepsilon \tau\left(q_{n}\right)^{2}, \forall h \in G_{0} \backslash E_{0}
$$

On the other hand, by Lemma 6.3 , for each $a_{g}^{h, n} \neq 0$ we have $\tau\left(\left|a_{g}^{h, n}\right|\right)=\tau\left(\sigma_{g}\left(q_{n}\right) q_{n}\right)$. Thus, since there are $\left|F_{n}^{h}\right|$-many such non-zero elements, by first using (6.5.4) and then (6.5.1) we get

$$
\begin{gathered}
|| F_{n}^{h}\left|-\tau(p)^{-1}\right|\left|\tau\left(q_{n}\right)^{2}=\right| F_{n}^{h}\left|\tau\left(q_{n}\right)^{2}-\tau(p)^{-1} \tau\left(q_{n}\right)^{2}\right| \\
\leq|| F_{n}^{h}\left|\tau\left(q_{n}\right)^{2}-\Sigma_{g} \tau\left(\sigma_{g}\left(q_{n}\right) q_{n}\right)\right|+\varepsilon \tau\left(q_{n}\right)^{2} \\
\leq\left(\left|F_{n}^{h}\right|+1\right) \varepsilon \tau\left(q_{n}\right)^{2} \leq\left(\tau(p)^{-1}+1\right) \varepsilon \tau\left(q_{n}\right)^{2} \leq\left(\varepsilon_{0} / 2\right) \tau\left(q_{n}\right)^{2} .
\end{gathered}
$$

Thus, $\tau(p)^{-1}$ is $\varepsilon_{0} / 2$ close to the integer $\left|F_{n}^{h}\right|$. By the choice of $\varepsilon_{0}$, this forces $\tau(p)^{-1}=\left|F_{n}^{h}\right|$.

We have thus proved that $\tau(p)^{-1}$ is an integer and that for each $n$ there exists $E_{0} \subset G_{0}$ finite such that for all $h \in G_{0} \backslash E_{0}$ we have $\left|F_{n}^{h}\right|=\tau(p)^{-1}$, while by 6.3. (ii) we also have $|\tau(p)-| c_{g}^{h}|| \leq 2^{-n+1} \tau(p), \forall g \in F_{n}^{h}$. Define $\tilde{G}=\left\{\left(u_{g_{n}}\right)_{n} \mid g_{n} \in\right.$ $G\} \subset \mathcal{U}\left(M^{\omega}\right)$ and notice that $L(\tilde{G})$ is naturally isomorphic to the von Neumann subalgebra of $M^{\omega}$ generated by $\tilde{G}$. Apply Lemma 5.3 to the set $\mathcal{W}$ of unitary elements in $p L(\tilde{G}) p$ which have Fourier expansion with $\tau(p)^{-1}$ non-zero coefficients, all of same absolute value $\tau(p)$. From the above remarks it follows that if $\left\{h_{n}\right\}_{n} \subset$ $G_{0}$ satisfies $h_{n} \rightarrow \infty$ then the class of $\left(u_{h_{n}}^{0}\right)_{n}$ in $M^{\omega}$ lies in $\mathcal{W}$. Since for all $h \in G_{0}$ we have $h h_{n} \rightarrow \infty$ as well, it follows that $\left(u_{h h_{n}}^{0}\right)_{n} \in \mathcal{W}, \forall h \in G_{0}$. But by $5.3 \mathcal{W}$ is a group, so the constant sequence $u_{h}^{0}=\left(u_{h h_{n}}^{0}\right)_{n}\left(u_{h_{n}}^{0}\right)_{n}^{*}$ lies in $\mathcal{W}, \forall h \in G_{0}$.

We have thus shown that the Fourier expansion of $u_{h}^{0}$ has exactly $\tau(p)^{-1}$ elements, all of absolute value $=\tau(p), \forall h \in G_{0}$. Thus, if we take $u=p$ then $(i),($ iii $)$ of 5.2 are consequences of Lemma 5.3. In other words, there exist a subgroup $G^{\prime} \subset G$, with a normal subgroup $K \subset G^{\prime}$ with $\tau(p)^{-1}$ elements and an $\operatorname{Ad}\left(G^{\prime}\right)$-invariant character $\kappa$ of $K$ such that $p=\Sigma_{k} \kappa(k) u_{k}$ and $\left\{u_{h}^{0}\right\}_{h}=\left\{u_{g} p \mid g \in G^{\prime}\right\}$, modulo scalars. 
In particular, this implies that $p$ is a Jones projection for the inclusion $A^{K} \subset A$, i.e. $p a p=E_{A^{K}}(a) p, \forall a \in A$, and there exists a projection $p_{0} \in A$ of trace $\tau\left(p_{0}\right)=$ $\tau(p)$ satisfying $p p_{0} p=\tau(p) p, A^{K} p_{0}=A p_{0}$.

To finish the proof of 5.2 we still need to show that $A_{0}=A^{K} p=p A p$. To this end note that (6.2) implies the following "approximation" of the equality $A_{0}\left(v^{*} q v\right)=$ $A^{K} q^{\prime} p$ in (5.4.1):

$$
\left\|v^{*} q v-\tau(p)^{-1} p q p\right\|_{2} \leq 2^{-n+1}\|q\|_{2}, \forall q \in \mathcal{P}\left(A q_{n}\right) .
$$

Moreover, since in particular $p q_{n} p$ is $2^{-n+1}$-close to a $\tau(p)$-multiple of a projection, for $n$ large enough it follows that $p q p$ must be equal to $\tau(p) q^{\prime}$ where $q^{\prime}=\tau(p)^{-1} E_{A^{K}}(q)$ is a projection in $A^{K}, \forall q \in \mathcal{P}\left(A q_{n}\right)$. Thus, with the above notations we may assume $q_{n} \leq p_{0}$ and

$$
\left\|v^{*} q v-q^{\prime} p\right\|_{2} \leq 2^{-n+1}\|q\|_{2}, \forall q \in \mathcal{P}\left(A q_{n}\right) .
$$

Let $\mathcal{F}=\left\{\left(e_{i}, h_{i}\right)\right\}_{i \in I} \subset \mathcal{P}\left(A q_{n}\right) \times G_{0}$ be a maximal family (with respect to inclusion) with the property that $\left\{\sigma_{\delta\left(h_{i}\right)}\left(e_{i}^{\prime}\right)\right\}_{i}$, resp. $\left\{\sigma_{0}\left(h_{i}\right)\left(v^{*} e_{i} v\right)\right\}_{i}$, are mutually orthogonal. If $f_{0}=\Sigma_{i} \sigma_{0}\left(h_{i}\right)\left(v^{*} e_{i} v\right) \neq p$ (equivalently $f=\Sigma_{i} \sigma_{\delta\left(h_{i}\right)}\left(e_{i}^{\prime}\right) \neq 1$ ), then by the ergodicity of $\sigma_{0}$ there exists $0 \neq e_{0} \in \mathcal{P}\left(A q_{n}\right) \subset A^{K} p_{0}$ and $h \in G_{0}$ such that $\sigma_{0}(h)\left(v^{*} e_{0} v\right) \leq p-f_{0}$. By the maximality of the family $\mathcal{F}$, it follows that $\sigma_{\delta(h)}\left(e_{0}\right) f \neq 0$. By shrinking $e_{0}$ if necessary, we may thus assume $\sigma_{\delta(h)}\left(e_{0}\right) \leq$ $\sigma_{\delta\left(h_{i}\right)}\left(e_{i}^{\prime}\right)$ for some $i \in I$. Thus, there exists $e_{i}^{0} \leq e_{i}$ in $\mathcal{P}\left(A q_{n}\right)$ such that

$$
\sigma_{\delta\left(h_{i}\right)}\left(e_{i}^{0}\right)=\sigma_{\delta(h)}\left(e_{0}\right), \sigma_{0}(h)\left(v^{*} e_{0} v\right) \sigma_{0}\left(h_{i}\right)\left(v^{*} e_{i}^{0} v\right)=0 .
$$

But since for $a \in A^{K}, a_{0} \in A_{0}$ we have $\sigma_{\delta(h)}(a) p=u_{\delta(h)}(a p) u_{\delta(h)}^{*}=u_{h}^{0} a u_{h}^{0 *}$ and $\sigma_{0}(h)\left(a_{0}\right)=u_{h}^{0} a_{0} u_{h}^{0 *}=u_{\delta(h)} a_{0} u_{\delta(h)}^{*}$, by (6.2) we get

$$
\left\|\sigma_{0}\left(h^{\prime}\right)\left(v^{*} q v\right)-\sigma_{\delta\left(h^{\prime}\right)}(q)\right\|_{2} \leq 2^{-n+1}, \forall q \in \mathcal{P}\left(A q_{n}\right), \forall h^{\prime} \in G_{0} .
$$

From (6.6.2) and (6.6.3) we finally get:

$$
\begin{gathered}
2^{1 / 2}\left\|e_{0}\right\|_{2}=\left\|\sigma_{0}(h)\left(v^{*} e_{0} v\right)-\sigma_{0}\left(h_{i}\right)\left(v^{*} e_{i}^{0} v\right)\right\|_{2} \\
\leq\left\|\sigma_{0}(h)\left(v^{*} e_{0} v\right)-\sigma_{\delta(h)}\left(e_{0}\right)\right\|_{2}+\left\|\sigma_{\delta\left(h_{i}\right)}\left(e_{i}^{0}\right)-\sigma_{0}\left(h_{i}\right)\left(v^{*} e_{i}^{0} v\right)\right\|_{2} \\
\leq 2^{-n+2}\left\|e_{0}\right\|_{2}
\end{gathered}
$$

which for $n \geq 2$ gives a contradiction. The maximal family $\mathcal{F}$ must therefore satisfy $\Sigma_{i} \sigma_{0}\left(h_{i}\right)\left(v^{*} e_{i} v\right)=p, \Sigma_{i} \sigma_{\delta\left(h_{i}\right)}\left(e_{i}^{\prime}\right)=1$. Since by $(6.2)$ we have $\left\|v^{*} e_{i}-e_{i}\right\|_{2} \leq$ $2^{-n}\left\|e_{i}\right\|_{2}$, if we define $V=\Sigma_{i} u_{h_{i}}^{0} e_{i} v u_{h_{i}}^{0 *}$ then clearly $V^{*} V=V V^{*}=p, V^{*}\left(A^{K} p\right) V=$ $A_{0}$ and

$$
\|V-p\|_{2}^{2}=\Sigma_{i}\left\|u_{h_{i}}^{0} e_{i} v u_{h_{i}}^{0 *}-u_{h_{i}}^{0} e_{i} u_{h_{i}}^{0 *}\right\|_{2}^{2} \leq \Sigma_{i} 2^{-n}\left\|e_{i}\right\|_{2}^{2}=2^{-n} .
$$

But since $n$ was arbitrary, this shows that $A^{K} p=A_{0}$. 


\section{Strong Rigidity Results}

The results we prove in this section will typically assume a "weak equivalence" between two free ergodic m.p. actions $\sigma: G \rightarrow \operatorname{Aut}\left(X_{0}, \mu_{0}\right), \sigma: G \rightarrow \operatorname{Aut}(X, \mu)$ satisfying certain conditions and will derive from that a much "stronger equivalence". There are two types of "weak equivalence" assumptions that we will consider:

Von Neumann equivalence (abbreviated $v N E$ ) of two actions $\sigma_{0}, \sigma$ is an isomorphism of the group measure space factors $M_{0}=L^{\infty}\left(X_{0}, \mu_{0}\right) \rtimes_{\sigma_{0}} G_{0}, M=$ $L^{\infty}(X, \mu) \rtimes_{\sigma} G$ associated with $\sigma_{0}, \sigma$. Most often we'll in fact consider its "stabilized version", i.e. isomorphism of some "corners" of these factors.

Orbit equivalence (abbreviated $O E$ ) of $\sigma_{0}, \sigma$, is an isomorphism $\Delta_{0}$ of the probability spaces $\left(X_{0}, \mu_{0}\right),(X, \mu)$ that takes the equivalence relation $\mathcal{R}_{\sigma_{0}}$ given by the orbits of $\sigma_{0}$ onto the equivalence relation $\mathcal{R}_{\sigma}$ given by the orbits of $\sigma$, almost everywhere. More generally we consider its "stabilized version", i.e. an isomorphism $\Delta_{0}:\left(X_{0}, \mu_{0}\right) \simeq\left(Y, \mu_{Y}\right)$, for some $Y \subset X$ with $\mu(Y) \neq 0$, that takes $\mathcal{R}_{\sigma_{0}}$ onto the equivalence relation $\mathcal{R}_{\sigma}^{Y}$ on $\left(Y, \mu_{Y}\right)$ given by the intersection of the orbits of $\sigma$ with the set $Y$.

Note that by $([\mathrm{Dy}],[\mathrm{FM}])$ an isomorphism $\Delta_{0}:\left(X_{0}, \mu_{0}\right) \simeq\left(Y, \mu_{Y}\right)$ extends to an algebra isomorphism $\theta_{0}: M_{0} \simeq p M p$, where $p=\chi_{Y}$, if and only if it takes onto each other the equivalence relations $\mathcal{R}_{\sigma_{0}}, \mathcal{R}_{\sigma}^{Y}$, i.e. if it is a (stable) OE. Furthermore, an isomorphism $\theta: M_{0} \simeq p M p$ comes from a (stable) OE iff it takes the subalgebra of coefficients $L^{\infty}\left(X_{0}, \mu_{0}\right), L^{\infty}(X, \mu) p$ (also called Cartan subalgebras) onto each other. Thus, $\mathrm{OE} \Rightarrow \mathrm{vNE}$ and any $\mathrm{OE}$ of actions can be viewed as a special type of vNE of the actions. Altogether, OE ergodic theory, which is the study of actions of groups up to orbit equivalence, is the same as the study of Cartan subalgebra inclusions $L^{\infty}(X, \mu) \subset L^{\infty}(X, \mu) \rtimes_{\sigma} G$ underlying the group measure space construction.

Conjugacy of actions is the typical "stronger equivalence" that we'll derive for $\sigma_{0}, \sigma$. This means an isomorphism of probability spaces $\Delta:\left(X_{0}, \mu_{0}\right) \simeq(X, \mu)$ satisfying $\left\{\Delta^{-1} \sigma_{g} \Delta\right\}_{g \in G}=\left\{\sigma_{0}(h)\right\}_{h \in G_{0}}$. In other words, $\sigma(\delta(h)) \Delta=\Delta \sigma_{0}(h)$, $\forall h \in G_{0}$, for some isomorphism of groups $\delta: G_{0} \simeq G$. Note that if $\Delta, \delta$ give a conjugacy of $\sigma_{0}, \sigma$, then they implement an isomorphism of the corresponding group measure space $\mathrm{II}_{1}$ factors $\theta^{\delta, \Delta}: L^{\infty}\left(X_{0}, \mu_{0}\right) \rtimes_{\sigma_{0}} G_{0} \simeq L^{\infty}(X, \mu) \rtimes_{\sigma} G$ by $\theta^{\delta, \Delta}\left(\Sigma_{h} a_{h}^{0} u_{h}^{0}\right)=\Sigma_{h} \Delta\left(a_{h}^{0}\right) u_{\delta(h)}$.

In one of the results though (Theorem 7.6), from vNE we'll merely derive OE, with only certain "parts" of $\left(\sigma_{0}, G_{0}\right),(\sigma, G)$ conjugate onto each other.

The conditions $\sigma_{0}, \sigma$ must satisfy are as follows: The "source" action $\left(\sigma_{0}, G_{0}\right)$ has to contain a sufficiently large subgroup satisfying the relative property ( $\mathrm{T}$ ) (of Kazhdan-Margulis [Ma]). The "target" action $(\sigma, G)$ needs to have good deforma- 
tion properties (malleability), examples of which are the Bernoulli $G$-actions. The precise definitions and notations, also used in ([Po4]), are as follows:

7.0.1. Definition. A group $G_{0}$ is w-rigid, if it contains an infinite normal subgroup $H_{0} \subset G_{0}$ with the relative property (T) of Kazhdan-Margulis ([Ma]; see also [dHV]).

7.0.2. Definition ([Po6]). An infinite subgroup $\Lambda$ of a group $\Gamma$ is weakly quasi normal (wq-normal) in $\Gamma$ if for any intermediate subgroup $\Lambda \subset H \varsubsetneqq \Gamma$ there exists $g \in \Gamma \backslash H$ such that $g H g^{-1} \cap H$ infinite. Note that $\Lambda$ is wq-normal in $\Gamma$ if and only if there exists an ordinal $\imath$ and a well ordered family of intermediate subgroups $\Lambda=H_{0} \subset H_{1} \subset \ldots \subset H_{\jmath} \subset \ldots \subset H_{\imath}=\Gamma$ such that for each $\jmath<\imath, H_{\jmath+1}$ is the group generated by the elements $g \in \Gamma$ with $g H_{\jmath} g^{-1} \cap H_{\jmath}$ infinite and such that if $\jmath \leq \imath$ has no "predecessor" then $H_{\jmath}=\cup_{n<\jmath} H_{n}$ (see [Po6]). It is easy to see that there exists a largest group $\Lambda \subset H^{\prime} \subset \Gamma$ such that $\Lambda$ is wq-normal in $H^{\prime}$, which we will call the wq-normalizer of $\Lambda$ in $\Gamma$.

If $\Lambda=H_{0} \subset H_{1} \subset \ldots \subset H_{n}=\Gamma$ are all normal inclusions, and $\Lambda$ is infinite, then $\Lambda \subset \Gamma$ is wq-normal. An inclusion of the form $\Lambda \subset\left(\Lambda * K_{0}\right) \times K_{1}$, with $\Lambda, K_{1}$ infinite $K_{0}$ arbitrary, is also wq-normal but cannot be realized as a sequence of consecutive normal inclusions.

7.0.3. Notation. We denote by $w \mathcal{T}_{0}$ the class of groups $G_{0}$ that contain an infinite, non-virtually abelian subgroup $H$ satisfying: $H \subset G_{0}$ has the relative property (T); $H$ is wq-normal in $G_{0}$.

7.0.4. Notation. We denote by $w \mathcal{T}_{1}$ the class of groups $G_{0}$ that have a w-rigid, wq-normal subgroup $H \subset G_{0}$ such that $\left\{h g h^{-1} \mid h \in H\right\}$ is infinite $\forall g \in G_{0}, g \neq e$. Note that if $G_{0}$ is itself w-rigid ICC then $G_{0} \in w \mathcal{T}_{1}$.

Note that all groups in $w \mathcal{T}_{1}$ are ICC (by the definition), while groups in $w \mathcal{T}_{0}$ are not required to be ICC. The class $w \mathcal{T}_{0}$ is clearly closed under normal and finite index extensions. It is also closed to inductive limits. All infinite property (T) groups $([\mathrm{Ka}])$ are both w-rigid and in the class $w \mathcal{T}_{0}$, and so are all groups of the form $\Gamma \times K$ with $\Gamma$ infinite property $(\mathrm{T})$ group and $K$ arbitrary. Also, if $\Gamma \in w \mathcal{T}_{0}$ then $\left(\Gamma * K_{0}\right) \times K_{1} \in w \mathcal{T}_{0}, \forall K_{0}$ arbitrary $K_{1}$ infinite. The groups $G=\mathbb{Z}^{2} \rtimes \Gamma$, with $\Gamma \subset S L(2, \mathbb{Z})$ non amenable are w-rigid (cf. [Ka], [Ma], [Bu]), and so are all the arithmetic groups in $([\mathrm{Va}],[\mathrm{Fe}])$. However, these groups are not in the class $w \mathcal{T}_{0}$ because in each of these cases the relatively rigid subgroup $\left(\mathbb{Z}^{N}\right.$, for some $\left.N\right)$ is abelian.

7.0.5. Definition. An action $\sigma$ of $G$ on $A \simeq L^{\infty}(X, \mu)$ is malleable (1.5 in [Po4]) if there exists a continuous action $\alpha: \mathbb{R} \rightarrow \operatorname{Aut}(A \bar{\otimes} A)$ that commutes with the "double" action $\sigma_{g} \otimes \sigma_{g}, g \in G$, and "flips" at some point $A \otimes 1$ onto $1 \otimes A$. It is 
$s$-malleable if there also exists $\beta \in \operatorname{Aut}(A \bar{\otimes} A)$ leaving $A=A \otimes 1$ pointwise fixed and satisfying $\beta^{2}=i d, \beta \alpha_{t}=\alpha_{-t} \beta, \forall t$. Also, $\sigma$ is sub malleable (resp. sub s-malleable) if it can be appropriately extended to a malleable (resp. s-malleable) action. For the precise definitions see (1.5 and 4.2 in [Po4]).

Given an arbitrary infinite discrete group $G$, an example of an action $\sigma$ of $G$ that is both sub s-malleable and clustering is the (left) Bernoulli shift action with "base space" $\left(Y_{0}, \nu_{0}\right)\left(Y_{0} \neq\right.$ single point). Recall that such $\sigma$ acts on $(X, \mu)=\Pi_{g}\left(Y_{0}, \nu_{0}\right)_{g}$ by $\sigma_{h}\left(\left(x_{g}\right)_{g}\right)=\left(x_{g}^{\prime}\right)_{g}$, where $x_{g}^{\prime}=x_{h^{-1} g}, \forall h, g \in G$. Recall also that a generalized Bernoulli shift action $\sigma$ of $G$ is defined as follows: Let $S$ be an infinite countable set and $\pi$ a faithful action of $G$ on $S$; let $(X, \mu)=\Pi_{s}\left(Y_{0}, \nu_{0}\right)_{s}$ and define the action $\sigma$ of $G$ on $(X, \mu)$ by $\sigma_{h}\left(\left(x_{s}\right)_{s}\right)=\left(x_{s}^{\prime}\right)_{s}$, where $x_{s}^{\prime}=x_{\pi\left(h^{-1}\right)(s)}, \forall h \in G, s \in S$. All generalized Bernoulli shift actions are sub s-malleable. They are mixing iff for any finite subset $S_{0} \subset S$ there exists $F \subset G$ finite such that $\pi(g) S_{0} \cap S_{0}=\emptyset, \forall g \in G \backslash F$. This condition is easily seen to also imply $\sigma$ is clustering.

To state the results, let us also recall that if $\gamma$ is a character of $G$ then it defines an automorphism $\theta^{\gamma}$ of $M=L^{\infty}(X, \mu) \rtimes_{\sigma} G$ by $\theta\left(\Sigma_{g} a_{g} u_{g}\right)=\Sigma_{g} \gamma(g) a_{g} u_{g}$, which is outer whenever $\gamma$ is non-trivial (see e.g. [T]).

7.1. Theorem (vNE Strong Rigidity). Let $\sigma: G \rightarrow \operatorname{Aut}(X, \mu), \sigma_{0}: G_{0} \rightarrow$ $\operatorname{Aut}\left(X_{0}, \mu_{0}\right)$ be free, ergodic actions of countable discrete groups $G, G_{0}$ on standard probability spaces $(X, \mu),\left(X_{0}, \mu\right)$. Denote $M=L^{\infty}(X, \mu) \rtimes_{\sigma} G, M_{0}=$ $L^{\infty}\left(X_{0}, \mu_{0}\right) \rtimes_{\sigma_{0}} G_{0}$. Assume:

(a). G is ICC; $\sigma$ is sub malleable clustering, e.g. $\sigma$ is a Bernoulli $G$-action;

(b). $G_{0}$ w-rigid ICC. More generally, $G_{0}$ has a w-rigid, wq-normal subgroup $H \subset G_{0}$ such that $\left\{h g h^{-1} \mid h \in G_{0}\right\}$ infinite $\forall e \neq g \in G_{0}$ and $\sigma_{0 \mid H}$ ergodic. (Note that $G_{0} \in w \mathcal{T}_{1}$.)

If $\theta: M_{0} \simeq p M p$ is an isomorphism, for some projection $p \in M$, then $p=1$ and there exist a character $\gamma$ of $G$ and isomorphisms $\delta: G_{0} \simeq G, \Delta:\left(X_{0}, \mu_{0}\right) \simeq(X, \mu)$ satisfying $\sigma(\delta(h)) \Delta=\Delta \sigma_{0}(h), \forall h \in G_{0}$, such that $\theta=\operatorname{Ad}(u) \circ \theta^{\gamma} \circ \theta^{\delta, \Delta}$ for some unitary $u \in M$.

Proof. Note first that if $H \subset G_{0}$ is as in condition (b) then $L(H)^{\prime} \cap M_{0}=\mathbb{C}$. This is because the condition $\left\{h g h^{-1} \mid h \in G_{0}\right\}$ infinite $\forall e \neq g \in G_{0}$ implies $L(H)^{\prime} \cap M_{0} \subset L^{\infty}\left(X_{0}, \mu_{0}\right)$ and since $H$ acts ergodically on $X_{0}$ this further implies $L(H)^{\prime} \cap M_{0} \subset\left(L^{\infty}\left(X_{0}, \mu_{0}\right)\right)^{H}=\mathbb{C}$.

We may clearly assume $p \in L(G)$. By (5.3 in [Po4]), there exists a unitary $u_{1} \in p M p$ such that $u_{1} \theta\left(L\left(G_{0}\right)\right) u_{1}^{*} \subset p L(G) p$. We may thus assume, for simplicity of notations, that $M_{0}=p M p$ with $p L(G) p \supset L\left(G_{0}\right)$. This allows us to apply Theorem 5.1. Since $G$ ICC implies $G$ has no non-trivial finite normal subgroups, 
it follows by 5.1 that $p=1$ and there exists a unitary element $u_{2} \in L(G)$ such that $\operatorname{Ad}\left(u_{2}\right)\left(u_{h}^{0}\right)=\gamma(\delta(h)) u_{\delta(h)}$, for some character $\gamma$ of $G$ and an isomorphism of groups $\delta: G_{0} \simeq G$. But this is equivalent to the required conclusions. Q.E.D.

7.1'. Theorem. With the same notations as in 7.1, if we assume:

(a). $G$ is ICC; $\sigma$ is sub s-malleable clustering, e.g. $\sigma$ is a Bernoulli G-action;

(b). $G_{0} \in w \mathcal{T}_{0}$.

then the same conclusion as in 7.1 holds true for any isomorphism $\theta: M_{0} \simeq p M p$.

Proof. The proof is exactly the same as for 7.1 , but using (5.3' in [Po4]) instead of (5.3 in $[\mathrm{Po} 4])$.

Q.E.D.

Taking in 7.1 (resp. $7.1^{\prime}$ ) both groups $G_{0}, G$ in $w \mathcal{T}_{1}$ (resp. in $w \mathcal{T}_{0}$ ) and both actions $\sigma_{0}, \sigma$ to be Bernoulli shifts, we get a 1 to 1 functor $G \mapsto L^{\infty}(X, \mu) \rtimes_{\sigma} G=M$ from the class $w \mathcal{T}_{0} \cup w \mathcal{T}_{1}$ (with group isomorphisms) to $\mathrm{II}_{1}$ factors (with algebra isomorphisms). Moreover, the same is true for the associated $\mathrm{II}_{\infty}$ factors $M^{\infty} \stackrel{\text { def }}{=}$ $M \bar{\otimes} \mathcal{B}\left(\ell^{2}(\mathbb{N})\right)$.

7.2. Corollary. Let $G_{i} \in w \mathcal{T}_{0} \cup w \mathcal{T}_{1}$ (e.g. w-rigid ICC) and $\sigma_{i}: G_{i} \rightarrow \operatorname{Aut}\left(X_{i}, \mu_{i}\right)$ be Bernoulli shift actions, $i=0,1$. Then the $\mathrm{II}_{1}$ factors $M_{i}=L^{\infty}\left(X_{i}, \mu_{i}\right) \rtimes_{\sigma_{i}} G_{i}$ (resp. $\mathrm{II}_{\infty}$ factors $M_{i}^{\infty}$ ), $i=0,1$, are isomorphic if and only if the groups $G_{i}$ are isomorphic and the actions $\sigma_{i}$ are conjugate, $i=0,1$. Even more so, if $\theta: M_{0}^{\infty} \simeq$ $M_{1}^{\infty}$, then $\theta$ is the amplification of an isomorphism $\theta_{0}: M_{0} \simeq M_{1}$ of the form $\theta_{0}=\operatorname{Ad}(u) \circ \theta^{\gamma} \circ \theta^{\delta, \Delta}$, for some $u \in \mathcal{U}\left(M_{1}\right), \gamma \in \operatorname{Char}\left(G_{1}\right)$ and $\delta: G_{0} \simeq G_{1}$, $\Delta:\left(X_{0}, \mu_{0}\right) \simeq\left(X_{1}, \mu_{1}\right)$ satisfying $\sigma_{1}(\delta(h)) \Delta=\Delta \sigma_{0}(h), \forall h \in G_{0}$.

For a Bernoulli shift action $\sigma$ of an ICC group $G, L^{\infty}(X, \mu) \rtimes_{\sigma} G$ can be viewed as the canonical "group measure space factor"-version of the "group factor" $L(G)$. Thus, Corollary 7.2 above can be regarded as an affirmative answer to a natural "relative variant" of Connes' rigidity conjecture ([C2]), stating that any isomorphism between factors arising from ICC property $(\mathrm{T})$ groups ought to come from an isomorphism of the groups. Note though that this "relative variant" holds true in a generality which, of course, fails to be true for group algebras $L(G)$. For instance given ANY ICC group $K$ (even $K$ amenable !) the group $G=S L(3, \mathbb{Z}) \times K$ is w-rigid, so the factors $L^{\infty}(X, \mu) \rtimes G$ are non-isomorphic for non-isomorphic $K$, by 7.2 , while the corresponding group factors $L(G)$ are mutually isomorphic if $K$ are taken amenable.

To state the next consequence, we need to recall some simple general facts about automorphism groups associated with actions, equivalence relations and von Neumann algebras. 
One denotes by $\operatorname{Aut}\left(\mathcal{R}_{\sigma}\right)$ the group of automorphisms of $(X, \mu)$ that preserve the orbits of $\sigma$ and by $\operatorname{Inn}\left(\mathcal{R}_{\sigma}\right)$ the subgroup of automorphism whose graph belongs to $\mathcal{R}_{\sigma}$. Equivalently, $\alpha \in \operatorname{Inn}\left(\mathcal{R}_{\sigma}\right)$ iff there exists $u$ in the normalizer of $A=L^{\infty}(X, \mu)$ in $M$ such that $\alpha=\operatorname{Ad}(u)_{\mid A}$. Note that $\operatorname{Aut}\left(\mathcal{R}_{\sigma}\right)$ is the normalizer of $\operatorname{Inn}\left(\mathcal{R}_{\sigma}\right)$ in $\operatorname{Aut}(X, \mu)$. We let $\operatorname{Out}\left(\mathcal{R}_{\sigma}\right)=\operatorname{Aut}\left(\mathcal{R}_{\sigma}\right) / \operatorname{Int}\left(\mathcal{R}_{\sigma}\right)$,

We denote by $\operatorname{Aut}(\sigma, G)$ the group of automorphisms $\Delta \in \operatorname{Aut}(X, \mu)$ for which there exists an (necessarily unique) automorphism $\delta=\delta(\Delta)$ of $G$ such that $\Delta \sigma_{g} \Delta^{-1}=$ $\sigma_{\delta(g)}, \forall g$. A particularly important subgroup of $\operatorname{Aut}(\sigma, G)$ is the commutant of $\sigma(G)$ in $\operatorname{Aut}(X, \mu)$, i.e. the group of automorphisms $\Delta \in \operatorname{Aut}(\sigma, G)$ for which $\delta(\Delta)=i d_{G}$. We denote this subgroup by $\operatorname{Aut}_{0}(\sigma, G)$. It is clearly normal in $\operatorname{Aut}(\sigma, G)$.

It is not hard to see that if $\Delta \in \operatorname{Aut}(\sigma, G)$ then $\Delta$ belongs to $\operatorname{Inn}\left(\mathcal{R}_{\sigma}\right)$ if and only if $\Delta=\sigma_{g}$ for some $g \in G$ (see e.g. [MoSo] or [Fu3]). Thus, the image of $\operatorname{Aut}(\sigma, G)$ in $\operatorname{Out}\left(\mathcal{R}_{\sigma}\right)$ coincides with the quotient $\operatorname{Out}(\sigma, G) \stackrel{\text { def }}{=} \operatorname{Aut}(\sigma, G) / \sigma(G)$.

Note that if $G$ is ICC then $\operatorname{Aut}_{0}(\sigma, G)$ is isomorphic to its image in $\operatorname{Aut}(\sigma, G) / \sigma(G)$ and thus to its image in $\operatorname{Out}\left(\mathcal{R}_{\sigma}\right)$ as well. Note also that if $\sigma$ is a Bernoulli shift then there is a canonical group embedding $\operatorname{Aut}(G) \ni \delta \mapsto \Delta_{\delta} \in \operatorname{Aut}(\sigma, G)$ given by $\Delta_{\delta}\left(\left(x_{g}\right)_{g}\right)=\left(x_{g}^{\prime}\right)_{g}$, where $x_{g}^{\prime}=x_{\delta^{-1}(g)}, \forall g \in G$. Thus, one has the split exact sequence

$$
\left.\left.1 \rightarrow \operatorname{Aut}_{0}(\sigma, G) \rightarrow \operatorname{Aut}(\sigma, G)\right) \rightarrow \operatorname{Aut}(\sigma, G)\right) / \operatorname{Aut}_{0}(\sigma, G)=\operatorname{Aut}(G) \rightarrow 1
$$

which in case $G$ is ICC gives the split exact sequence:

$$
\left.1 \rightarrow \operatorname{Aut}_{0}(\sigma, G) \rightarrow \operatorname{Aut}(\sigma, G)\right) / \sigma(G) \rightarrow \operatorname{Out}(G) \rightarrow 1
$$

Finally, note that if we put $M=L^{\infty}(X, \mu) \rtimes_{\sigma} G$ and denote as usual $\operatorname{Out}(M)=$ $\operatorname{Aut}(M) / \operatorname{Int}(M)$, then we have a natural embedding $\operatorname{Aut}\left(\mathcal{R}_{\sigma}\right) \subset \operatorname{Aut}(M)$ whose image in $\operatorname{Out}(M)$ is $\operatorname{Out}\left(\mathcal{R}_{\sigma}\right)$. Another remarkable subgroup of $\operatorname{Aut}(M)$ related to the action $\sigma$ is the group $\left\{\theta^{\gamma} \mid \gamma \in \operatorname{Char}(G)\right\} \simeq \operatorname{Char}(G)$, with $\theta^{\gamma} \in \operatorname{Aut}(M)$ as defined at the beginning of this section. This group is isomorphic to its image in $\operatorname{Out}(M)$ and it is normalized by the image of $\operatorname{Aut}(\sigma, G)$ in $\operatorname{Aut}(M)$, with their intersection being trivial.

With these remarks in mind, and denoting as before $M^{\infty}=M \bar{\otimes} \mathcal{B}\left(\ell^{2}(\mathbb{N})\right)$ the type $\mathrm{II}_{\infty}$ factor associated with $M$, Corollary 7.2 trivially implies:

7.3. Corollary. With the above notations, assume $G \in w \mathcal{T}_{0} \cup w \mathcal{T}_{1}$ (e.g. $G$ wrigid $I C C)$ and $\sigma: G \rightarrow \operatorname{Aut}(X, \mu)$ is a Bernoulli $G$-action. Then $\operatorname{Out}\left(M^{\infty}\right)=$ $\operatorname{Out}(M)=\operatorname{Aut}_{0}(\sigma, G) \times(\operatorname{Char}(G) \rtimes \operatorname{Out}(G))$. In particular, $\mathscr{F}(M)=\{1\}$. Also, $\operatorname{Out}\left(\mathcal{R}_{\sigma}\right)=\operatorname{Aut}_{0}(\sigma, G) \times \operatorname{Out}(G)$ and $\mathscr{F}\left(\mathcal{R}_{\sigma}\right)=\{1\}$. 
The above corollary reduces the calculation of the outer automorphism group $\operatorname{Out}(M)$ of $\mathrm{II}_{1}$ factors $M=L^{\infty}(X, \mu) \rtimes_{\sigma} G$ (resp. equivalence relations $\mathcal{R}_{\sigma}$ ) with $G$ ICC group in the class $w \mathcal{T}_{0} \cup w \mathcal{T}_{1}$ (e.g. for $G$ w-rigid ICC group) and $\sigma$ Bernoulli shift of base $\left(Y_{0}, \mu_{0}\right)$, to the calculation of the commutant $\operatorname{Aut}_{0}(\sigma, G)=\{\theta \in$ $\left.\operatorname{Aut}(X, \mu) \mid \theta \sigma_{g}=\sigma_{g} \theta, \forall g \in G\right\}$ of the left Bernoulli shift $\sigma$. We conjecture that any $\theta$ commuting with $\sigma$ must be of the form $\theta=\theta_{0} \theta_{1}$ with $\theta_{0}$ a right shift by some element of the group $G$ and $\theta_{1}$ a product type action $\theta_{1}=\Pi_{g} \alpha_{g}$, where $\alpha_{g}=\alpha, \forall g$, for some $\alpha \in \operatorname{Aut}\left(Y_{0}, \mu_{0}\right)$. Note that if this would be the case, then $\operatorname{Aut}_{0}(\sigma, G) \simeq G \times \operatorname{Aut}\left(Y_{0}, \mu_{0}\right)$, so by 7.3 it would follow that $\operatorname{Out}(M)=G \times$ $\operatorname{Aut}\left(Y_{0}, \mu_{0}\right) \times(\operatorname{Char}(G) \rtimes \operatorname{Out}(G))$ and $\operatorname{Out}\left(\mathcal{R}_{\sigma}\right)=G \times \operatorname{Aut}\left(Y_{0}, \mu_{0}\right) \times \operatorname{Out}(G)$. Such a calculation can be shown to imply that two Bernoulli shift actions $\sigma_{0}, \sigma_{1}$ of ICC groups $G_{0}, G_{1} \in w \mathcal{T}_{0} \cup w \mathcal{T}_{1}$ with base space $\left(Y_{0}, \nu_{0}\right),\left(Y_{1}, \nu_{1}\right)$ give rise to isomorphic $\mathrm{II}_{1}$ group measure space factors $M_{0}, M_{1}$ iff $G_{0} \simeq G_{1}$ and $\left(Y_{0}, \nu_{0}\right) \simeq\left(Y_{1}, \nu_{1}\right)$.

The "weak-normality" assumptions on the relatively rigid subgroup of $G_{0}$ in 7.1.(b), 7.1'.(b) allowed us to obtain plain conjugacy of actions from von Neumann isomorphism (vNE). Using the full generality of the Cartan Conjugacy Criteria in Section 4, one can still obtain significant information (notably OE equivalence from vNE equivalence) even if we drop such weak normality assumption:

7.4. Theorem (vNE/OE Strong Rigidity). Let $\sigma: G \rightarrow \operatorname{Aut}(X, \mu), \sigma_{0}: G_{0} \rightarrow$ $\operatorname{Aut}\left(X_{0}, \mu_{0}\right)$ be free ergodic m.p. actions. Denote $A=L^{\infty}(X, \mu), A_{0}=L^{\infty}\left(X_{0}, \mu_{0}\right)$, $M=A \rtimes_{\sigma} G, M_{0}=A_{0} \rtimes_{\sigma_{0}} G_{0}$. Assume:

(a). G is ICC; $\sigma$ is sub s-malleable clustering, e.g. $\sigma$ Bernoulli $G$-action.

(b). $G_{0}$ has a non virtually abelian subgroup $H \subset G_{0}$ with the relative property $(\mathrm{T})$.

If $\theta: M_{0} \simeq p M p$ is an isomorphism, for some projection $p \in M$, then $\tau(p)^{-1}$ is an integer and there exists a partial isometry $v \in p M p$ such that

(i). $v^{*} v=p, v v^{*} \in A, v \theta\left(A_{0}\right) v^{*}=A v v^{*}$.

(ii). If $G_{0}^{\prime} \subset G_{0}$ is the wq-normalizer of $H$ in $G_{0}$, then there exists $G^{\prime} \subset G$ and $K \triangleleft G^{\prime},|K|=\tau(p)^{-1}$, such that $\operatorname{Ad}(v) \circ \theta$ conjugates $\left(\sigma_{0}\right)_{\mid G_{0}^{\prime}}$ onto the action $\sigma^{\prime}$ of $G^{\prime} / K$ on $A^{K}=\left\{a \in A \mid \sigma_{k}(a)=a, \forall k \in K\right\}$ given by $\sigma^{\prime}\left(\hat{g^{\prime}}\right)=\left(\sigma_{g^{\prime}}\right)_{\mid A^{K}}, \forall g^{\prime} \in G^{\prime}$.

Proof. Like in the proofs of 7.1, 7.1', by ([Po4]) we may assume $p \in L(G)$ and $\theta\left(L\left(G_{0}^{\prime}\right)\right) \subset p L(G) p$. By Theorem 4.2 it follows that $\theta\left(A_{0}\right)$ is unitary conjugate to $A p_{0}$ for some projection $p_{0} \in A$ with $\tau\left(p_{0}\right)=\tau(p)$. But then 5.2 applies to get the conclusion.

Q.E.D.

The first result deriving OE (equivalently, Cartan conjugacy) from vNE, i.e. from the isomorphism of the group measure space factors, was obtained in $([\mathrm{Po} 2])$ : It is shown there that if $M_{0}=M\left(\mathcal{R}_{0}\right), M=\mathcal{M}(\mathcal{R})$ for some equivalence relations 
$\mathcal{R}_{0}, \mathcal{R}$, with $\mathcal{R}_{0}$ having the relative property ( $\mathrm{T}$ ) (as defined in 5.10.1 in [Po2]) and $\mathcal{R}$ having the Haagerup property (as considered in 2.1 and 3.5.6 of [Po2]) then any isomorphism $\theta: M_{0} \simeq M$ is an inner perturbation of an isomorphism coming from OE. Once such a "vNE/OE Strong Rigidity" is proved, one can take advantage of results on $\mathrm{OE}$ Ergodic Theory to derive results about the $\mathrm{II}_{1}$ factors involved. Thus, in ([Po2]) one uses Gaboriau's $\ell^{2}$-Betti numbers for equivalence relations to derive invariants for the corresponding group measure space factors, (called the class $\mathcal{H} \mathcal{T})$.

Similarly, one can use 7.4 in combination with ([Ga2]), or with the MonodShalom OE Strong Rigidity results in ([MoSh]), to obtain calculation of fundamental groups and non-isomorphism of $\mathrm{II}_{1}$ group measure space factors that are not covered by Theorem 7.1. For instance, one can apply 7.4 to the case $G_{0}, G$ are products of two or more groups of the form $H * K$ with $H, K$ torsion free and $H$ Kazhdan, to get OE from vNE, then apply ([MoSh] $)$ to further get conjugacy from $\mathrm{OE}$, and the triviality of the fundamental group. Along these lines, we mention a consequence of 7.4 which derives the triviality of fundamental group of certain factors without using OE rigidity results from ([Ga2], [MoSh]):

7.5. Corollary. If $G$ is an ICC group having a non virtually abelian subgroup with the relative property (T) (e.g. $G=H * K$ for some infinite Kazhdan group $H$ and $K \neq 1$, or a product of such groups) and $\sigma$ is a Bernoulli G-action, then the fundamental group of the corresponding group measure space factor $M$ satisfies $\mathscr{F}(M) \cap[1, \infty) \subset \mathbb{N}$. If in addition $G$ is torsion free (e.g. $G=H * K$ with $H$ Kazhdan and $H, K$ torsion free) then $\mathscr{F}(M)=\{1\}$.

Since any OE of actions entails an isomorphism of the associated group measure space factors, Theorems 7.1, 7.1' trivially imply OE rigidity results for actions $\sigma, \sigma_{0}$ satisfying conditions $(a),(b)$ of either 7.1 or 7.1 '. But in fact we can do slightly better than that: Since an isomorphism of group measure space factors that comes from an $\mathrm{OE}$ isomorphism of probability spaces takes Cartan subalgebras onto each other, we can use 5.2 (which only requires mixing conditions on the actions) rather than 5.1 (which is what we used in the proofs of 7.1, 7.1', and which required the clustering condition on $\sigma$ ). This allows us to replace the condition " $\sigma$ clustering" by the weaker condition " $\sigma$ mixing".

7.6. Theorem (OE Strong Rigidity). Let $\sigma: G \rightarrow \operatorname{Aut}(X, \mu), \sigma_{0}: G_{0} \rightarrow$ $\operatorname{Aut}\left(X_{0}, \mu_{0}\right)$ be free ergodic m.p. actions. Assume:

(a). G is ICC; $\sigma$ is sub s-malleable and mixing (e.g. a Bernoulli G-action).

(b). Either $G_{0} \in w \mathcal{T}_{0}$; or $\left(\sigma_{0}, G_{0}\right)$ satisfies $7.1 .(b)$ (e.g., $G_{0}$ w-rigid ICC) 
If $Y \subset X$ is a subset of positive measure and $\Delta_{0}:\left(X_{0}, \mu_{0}\right) \simeq\left(Y, \mu_{Y}\right)$ satisfies $\Delta_{0}\left(\mathcal{R}_{\sigma_{0}}\right)=\mathcal{R}_{\sigma}^{Y}$ then $Y=X$, the groups $G_{0}, G$ are isomorphic and the actions $\sigma_{0}, \sigma$ are conjugate with respect to that isomorphism of groups. More precisely, there exist $\alpha \in \operatorname{Inn}\left(\mathcal{R}_{\sigma}\right), \delta: G_{0} \simeq G, \Delta:\left(X_{0}, \mu_{0}\right) \simeq(X, \mu)$ satisfying $\sigma(\delta(h))(\Delta(x))=$ $\Delta\left(\sigma_{0}(h)(x)\right), \forall h \in G_{0}, x \in X_{0}$, such that $\Delta_{0}=\alpha \circ \Delta$.

Proof. With the usual notation $M_{0}, M$ for the associated group measure space factors, the isomorphism $\Delta_{0}$ implements an isomorphism $\theta: M_{0} \simeq p M p$ satisfying $\theta\left(A_{0}\right)=A p$, where $p=\chi_{Y}$. Since $L(G)$ is diffuse (because $G$ is infinite), $p$ can be unitary conjugate to a projection in $L(G)$. Equivalently, after identifying $M_{0}$ with $\theta\left(M_{0}\right)$, we may assume $M_{0}=p M p$ for some projection $p \in L(G)$. By applying (5.3 in [Po4]), in the case $\left(\sigma_{0}, G_{0}\right)$ satisfies $7.1 .(b)$, and respectively (5.3' in [Po4]) in the case $G_{0} \in w \mathcal{T}_{1}$, it follows that we may also assume $L\left(G_{0}\right) \subset p L(G) p$. Thus, assumptions $(a)$ and $(c)$ of 5.2 are satisfied. Since $G$ is ICC and $M_{0}=p M p$, all the conditions required in the last part of 5.2 are checked and the corresponding conclusion in 5.2 trivially implies the statement.

Q.E.D.

Following ([MoSh]), to have an "OE superrigidity"-type result one needs all conditions on just one side. Theorem 7.6, which has the conditions on the group (either w-rigid ICC or in the class $w \mathcal{T}_{0}$ ) on the "source" side and the conditions on the action (Bernoulli) on the "target" side, doesn't quite fulfill this requirement. By strengthening the condition on the group though, we can obtain this kind of result as well:

7.7. Theorem (OE Superrigidity). Let $G$ be an ICC property $(\mathrm{T})$ group and $\sigma: G \rightarrow \operatorname{Aut}(X, \mu)$ a Bernoulli $G$-action (more generally, a sub s-malleable, mixing action). Let $G_{0}$ be any group and $\sigma_{0}: G_{0} \rightarrow \operatorname{Aut}\left(X_{0}, \mu_{0}\right)$ any free m.p. action of $G_{0}$. If $\Delta_{0}:\left(X_{0}, \mu_{0}\right) \simeq\left(Y, \mu_{\mid Y}\right)$ satisfies $\Delta_{0}\left(\mathcal{R}_{\sigma_{0}}\right)=\mathcal{R}_{\sigma}^{Y}$, for some subset $Y \subset X$, then $Y=X$ (modulo null sets), the groups $G_{0}, G$ are isomorphic and the actions $\sigma_{0}, \sigma$ are conjugate with respect to that isomorphism of groups. More precisely, there exist $\alpha \in \operatorname{Inn}\left(\mathcal{R}_{\sigma}\right), \delta: G_{0} \simeq G$ such that $\Delta=\alpha \circ \Delta_{0}$ satisfies $\sigma(\delta(h)) \Delta=\Delta \sigma_{0}(h)$, $\forall h \in G_{0}$.

Proof. By either (4.1.7, 4.1.9 in [Po8]) or (1.4 in [Fu2]), if $\mathcal{R}_{\sigma_{0}} \simeq \mathcal{R}_{\sigma}^{Y}$ and $G$ has property $(\mathrm{T})$ then $G_{0}$ has property $(\mathrm{T})$. But then we can apply 7.5 and the statement follows.

Q.E.D.

Note that the class of groups and actions covered by the OE rigidity results 7.6 and 7.7 is quite different from the ones in ([Fu1], [MoSh] $)$. However, some intersection occurs with $([\mathrm{MoSh}])$. For instance, if we take $G_{0}=\Gamma_{1} \times \ldots \times \Gamma_{n}$ with $n \geq 2$, each $\Gamma_{i}$ either a free product of torsion free groups or a non-elementary 
Gromov-hyperbolic group with the property $(\mathrm{T})$, at least one being of this latter class, then each $\Gamma_{i}$ is in the class $\mathcal{C}$ of Monod-Shalom while $G_{0}$ is weakly rigid. Thus, if the action $\sigma_{0}$ of $\Gamma_{1} \times \ldots \times \Gamma_{n}$ is assumed irreducible, in the sense of ([MoSh]), and $\sigma$ is Bernoulli with $G$ ICC, then the corresponding OE rigidity result is covered by both 7.5 above and (1.9 in [MoSh]). On the other hand, we were not able to check Furman's condition for Bernoulli shift actions $\sigma$ of higher rank lattices $\Gamma \subset \mathcal{G}$, i.e. to show whether $(\sigma, G ; X)$ has no quotients of the form $\mathcal{G} / \Lambda$, with $\Lambda$ a lattice in $\mathcal{G}$. If this would be true (which is what we expect), then (Theorem $\mathrm{A}$ in [Fu1]) would imply: Let $G$ be an ICC higher rank lattice and $\sigma$ Bernoulli $G$-action. If $\mathcal{R}_{\sigma}^{Y} \simeq \mathcal{R}_{\sigma_{0}}$ for some $Y \subset X$ and some free ergodic m.p. action $\left(\sigma_{0}, G_{0}\right)$, then $G, G_{0}$ are "virtually isomorphic" groups and $\sigma, \sigma_{0}$ "virtually conjugate" actions.

Our next result gives a Galois type correspondence for the w-rigid ICC subequivalence relations of an equivalence relation implemented by a Bernoulli shift. The result can also be viewed as a OE Strong Rigidity result for embeddings of equivalence relations.

7.7. Theorem (OE Strong Rigidity for Embeddings. Let $\sigma: G \rightarrow \operatorname{Aut}(X, \mu)$ be a Bernoulli $G$-action (or more generally a sub s-malleable mixing m.p. action) of an ICC group $G$. If $\sigma_{0}: G_{0} \rightarrow \operatorname{Aut}\left(X_{0}, \mu_{0}\right)$ is a free ergodic m.p. action of a group $G_{0}$ which is either w-rigid ICC or in the class $w \mathcal{T}_{0}$ and $\Delta_{0}:\left(X_{0}, \mu_{0}\right) \simeq$ $(X, \mu)$ satisfies $\Delta_{0}\left(\mathcal{R}_{\sigma_{0}}\right) \subset \mathcal{R}_{\sigma}$ then there exists an isomorphism $\delta: G_{0} \simeq G^{\prime} \subset G$ and $\alpha \in \operatorname{Inn}\left(\mathcal{R}_{\sigma}\right)$ such that $\alpha \circ \Delta_{0} \circ \sigma_{0}(h)=\sigma(\delta(h)), \forall h \in G_{0}$. Thus, there is a 1 to 1 correspondence between the sub-equivalence relations of $\mathcal{R}_{\sigma}$ that are implementable by free ergodic actions of w-rigid ICC groups (resp. groups in $w \mathcal{T}_{0}$ ), modulo conjugacy by automorphisms in $\operatorname{Inn}\left(\mathcal{R}_{\sigma}\right)$, and the w-rigid ICC subgroups of $G$ (resp. the $w \mathcal{T}_{0}$ subgroups of $G$ ), modulo conjugacy by elements in $G$.

Proof. We may assume $\mathcal{R}_{\sigma_{0}} \subset \mathcal{R}_{\sigma}$, i.e. we can select unitaries $\left\{u_{h}^{0}\right\}_{h \in G_{0}}$ in the normalizer of $A=L^{\infty}(X, \mu)$ in $M=L^{\infty}(X, \mu) \rtimes_{\sigma} G$ such that $G_{0} \ni h \mapsto \sigma_{0}(h)=$ $\operatorname{Ad}\left(u_{h}^{0}\right) \in \operatorname{Aut}(X, \mu), \forall h \in G_{0}$. Since $A \subset M=M\left(\mathcal{R}_{\sigma}\right)$ has vanishing 2-cohomology in the sense of $([\mathrm{FM}])$, it follows that for any sub-equivalence $\mathcal{R} \subset \mathcal{R}_{\sigma}$ the Cartan inclusion $A \subset M(\mathcal{R})$ has vanishing 2-cocycle. Thus, by perturbing if necessary each $u_{h}^{0}$ by a unitary element in $\mathcal{U}(A)$ we may assume $\left\{u_{h}^{0}\right\}_{h \in G_{0}}$ are the canonical unitaries generating $L\left(G_{0}\right)$. If $G_{0}$ is w-rigid ICC then by (5.3 in [Po4]) there exists a unitary element $u$ in $M$ such that $u L\left(G_{0}\right) u^{*} \subset L(G)$. Since $\sigma$ is clustering, by Lemma 4.5 it follows that $\sigma_{0}$ is mixing, thus Theorem 5.2 can be applied to conclude that there exists $u \in \mathcal{U}(M)$ such that $u\left(\left\{u_{h}^{0}\right\}_{h}\right) u^{*} \subset \mathbb{T}\left\{u_{g}\right\}_{g}$ and $u A u^{*}=A$. The proof of the case $G_{0} \in w \mathcal{T}_{0}$ is similar, using (5.3' in [Po4]).

Q.E.D.

In (part (1) of Theorem D in [Fu1]), Alex Furman proved that the restrictions to subsets $Y$ of irrational measure of the equivalence relation $\mathcal{R}_{\sigma}$ given by a classical 
action $\sigma$ of an arithmetic Kazhdan group, such as $S L(n, \mathbb{Z})$ on $\mathbb{T}^{n}, n \geq 3$, cannot be implemented by a free action of a group. We end by noticing that Theorem 7.7 provides a new class of examples of this situation, without restrictions on the measure of the subsets. Note however that (Thm. D(3) in [Fu1]) also provides examples of equivalence relations $\mathcal{R}$ with the property that no amplification $\mathcal{R}^{t}, t>$ 0 can be implemented by a free action of a group, a situation which we cannot realize here (see however Remark 5.6.2 in [Po4]) !

7.8. Corollary. Let $\sigma: G \rightarrow \operatorname{Aut}(X, \mu)$ be a Bernoulli shift action (or more generally a free, sub s-malleable, mixing m.p. action) of an ICC property (T) group $G$. If $Y \subset X$ has measure $0 \neq \mu(Y)<1$ then $\mathcal{R}_{\sigma}^{Y}$ (the restriction of the equivalence relation $\mathcal{R}_{\sigma}$ to $Y$ ) cannot be realized as orbits of a free action of a group.

\section{REFERENCES}

[Bu] M. Burger: Kazhdan constants for $\mathrm{SL}(3, \mathbb{Z})$, J. reine angew. Math., 413 (1991), 36-67.

[Chr] E. Christensen: Subalgebras of a finite algebra, Math. Ann. 243 (1979), 17-29.

[C1] A. Connes: A type $I_{1}$ factor with countable fundamental group, J. Operator Theory 4 (1980), 151-153.

[C2] A. Connes: Classification des facteurs, Proc. Symp. Pure Math. 38 (Amer. Math. Soc. 1982), 43-109.

[C3] A. Connes: Classification of injective factors, Ann. of Math., 104 (1976), 73-115.

[C4] A. Connes: Sur la classification des facteurs de type II, C. R. Acad. Sci. Paris 281 (1975), 13-15.

[CJ1] A. Connes, V.F.R. Jones: A $\mathrm{II}_{1}$ factor with two non-conjugate Cartan subalgebras, Bull. Amer. Math. Soc. 6 (1982), 211-212.

[CJ2] A. Connes, V.F.R. Jones: Property (T) for von Neumann algebras, Bull. London Math. Soc. 17 (1985), 57-62.

[CSh] A. Connes, D. Shlyakhtenko: $L^{2}$-homology for von Neumann algebras, preprint, math.OA/0309343.

[CoHa] M. Cowling, U. Haagerup: Completely bounded multipliers of of the Fourier algebra of a simple Lie group of real rank one, Invent. Math. 96 (1989), 507549.

[CoZi] M. Cowling, R. Zimmer: Actions of lattices in $S p(n, 1)$, Ergod. Th. Dynam. Sys. 9 (1989), 221-237.

[Dy] H. Dye: On groups of measure preserving transformations, II, Amer. J. Math, 85 (1963), 551-576.

[FM] J. Feldman, C.C. Moore: Ergodic equivalence relations, cohomology, and von 
Neumann algebras I, II, Trans. Amer. Math. Soc. 234 (1977), 289-324, 325359.

[Fe] T. Fernos: Kazhdan's Relative Property (T): Some New Examples, math.GR/0411527.

[Fu1] A. Furman: Orbit equivalence rigidity, Ann. of Math. 150 (1999), 1083-1108.

[Fu2] A. Furman: Gromov's measure equivalence and rigidity of higher rank lattices, Ann. of Math. 150 (1999), 1059-1081.

[Fu3] A. Furman: Outer automorphism groups of some ergodic equivalence relations, preprint 2003.

[Ga1] D. Gaboriau: Cout des rélations d'équivalence et des groupes, Invent. Math. 139 (2000), 41-98.

[Ga2] D. Gaboriau: Invariants $\ell^{2}$ de rélations d'équivalence et de groupes, Publ. Math. I.H.É.S. 95 (2002), 93-150.

[GeGo] S. Gefter, V. Golodets: Fundamental groups for ergodic actions and actions with unit fundamental groups, Publ. RIMS, Kyoto Univ. 24 (1988), 821-847.

[GoNe] V. Y. Golodets, N. I. Nesonov: T-property and nonisomorphic factors of type II and III, J. Funct. Analysis 70 (1987), 80-89.

[Gr] M. Gromov: Hyperbolic groups in "Essays in group theory" (S.M.S. Gersten editor), Springer, 1987, 75-263.

[Ha] U. Haagerup: An example of a non-nuclear $C^{*}$-algebra which has the metric approximation property, Invent. Math. 50 (1979), 279-293.

[Hj] G. Hjorth: A converse to Dye's theorem, UCLA preprint, Sept. 2002.

[dHV] P. de la Harpe, A. Valette: "La propriété T de Kazhdan pour les groupes localement compacts", Astérisque 175 (1989).

[J1] V.F.R. Jones : Index for subfactors, Invent. Math. 72 (1983), 1-25.

[J2] V.F.R. Jones : Ten problems, in "Mathematics: perspectives and frontieres", pp. 79-91, AMS 2000, V. Arnold, M. Atiyah, P. Lax, B. Mazur Editors.

[Ka] D. Kazhdan: Connection of the dual space of a group with the structure of its closed subgroups, Funct. Anal. and its Appl., 1 (1967), 63-65.

[Ma] G. Margulis: Finitely-additive invariant measures on Euclidian spaces, Ergodic. Th. and Dynam. Sys. 2 (1982), 383-396.

[MoSh] N. Monod, Y. Shalom: Orbit equivalence rigidity and bounded cohomology, Preprint 2002.

[Moo] C.C. Moore: Ergodic theory and von Neumann algebras, Proc. Symp. Pure Math. 38 (Amer. Math. Soc. 1982), 179-226.

[MvN1] F. Murray, J. von Neumann: On rings of operators, Ann. Math. 37 (1936), 116-229.

[MvN2] F. Murray, J. von Neumann: Rings of operators IV, Ann. Math. 44 (1943), 716-808. 
[NeSt] S. Neshveyev, E. Størmer: Ergodic theory and maximal abelian subalgebras of the hyperfinite factor, JFA 195 (2002), 239-261.

[Oz] N. Ozawa: A Kurosh type theorem for type $\mathrm{II}_{1}$ factors, math.OA/0401121.

[Po1] S. Popa: Some rigidity results for non-commutative Bernoulli shifts, MSRI preprint 2001-005.

[Po2] S. Popa: On a class of type $I I_{1}$ factors with Betti numbers invariants, MSRI preprint 2001-024, revised math.OA/0209310, to appear in Ann. of Math.

[Po3] S. Popa: On the fundametal group of type $\mathrm{II}_{1}$ factors, Proc. Nat. Acad. Sci. 101 (2004), 723-726. (math.OA/0210467).

[Po4] S. Popa: Strong rigidity of $\mathrm{II}_{1}$ factors arising from malleable actions of weakly rigid groups, I, math.OA/0305306.

[Po5] S. Popa: On the distance between MASA's in type $\mathrm{II}_{1}$ factors, Fields Institute Comm. 30 (2001), 321-324.

[Po6] S. Popa: Some computations of 1-cohomology groups and construction of non orbit equivalent actions, math.OA/0407199.

[Po7] S. Popa: Orthogonal pairs of *-subalgebras in finite von Neumann algebras, Journal of Operator Theory, 9 (1983), 253-268.

[Po8] S. Popa: Correspondences, INCREST preprint 1986.

[Po9] S. Popa: Classification of subfactors of type II, Acta Math. 172 (1994), 163-255.

[PoSa] S. Popa, R. Sasyk: On the cohomology of actions of groups by Bernoulli shifts, math.OA/0311417.

[PoSiSm] S. Popa, A. Sinclair, R. Smith: Perturbations of subalgebras of type $\mathrm{II}_{1}$ factors, J. Funct. Analysis 213 (2004), 346-379 (math.OA/0305444).

[S] I.M. Singer: Automorphisms of finite factors, Amer. J. Math. 177 (1955), 117-133.

[T] M. Takesaki: "Theory of Operator Algebras II", Encyclopedia of Mathematical Sciences 125, Springer-Verlag, Berlin-Heidelberg-New York, 2002.

[Va] A. Valette: Group pairs with relative property $(\mathrm{T})$ from arithmetic lattices, preprint 2004 (preliminary version 2001).

[Zi] R. Zimmer: "Ergodic theory and semisimple groups", Birkhaüser-Verlag, Boston 1984.

MAтh.Dept., UCLA, LA, CA 90095-155505

E-mail address: popa@math.ucla.edu 